J. Inst. Math. Jussieu (2023), 22(2), 681-746

doi:10.1017/S147474802100027X C The Author(s), 2021. Published by Cambridge University

Press. This is an Open Access article, distributed under the terms of the Creative Commons

Attribution licence (http://creativecommons.org/licenses/by/4.0/), which permits unrestricted re-use, distribution and reproduction, provided the original article is properly cited.

\title{
EULER CLASSES: SIX-FUNCTORS FORMALISM, DUALITIES, INTEGRALITY AND LINEAR SUBSPACES OF COMPLETE INTERSECTIONS
}

\author{
TOM BACHMANN (10 ${ }^{1}$ AND KIRSTEN WICKELGREN (10 2 \\ ${ }^{1}$ Mathematisches Institut, LMU Munich, Theresienstr. 39, D-80333 München, Germany \\ (tom.bachmann@zoho.com) \\ ${ }^{2}$ Department of Mathematics, Duke University, 120 Science Drive, 117 Physics \\ Building, Campus Box 90320, Durham, NC 27708-032, USA \\ (kirsten.wickelgren@duke.edu)
}

(Received 11 February 2020; revised 19 May 2021; accepted 20 May 2021; first published online 16 July 2021)

\begin{abstract}
We equate various Euler classes of algebraic vector bundles, including those of [12] and one suggested by M. J. Hopkins, A. Raksit, and J.-P. Serre. We establish integrality results for this Euler class and give formulas for local indices at isolated zeros, both in terms of the six-functors formalism of coherent sheaves and as an explicit recipe in the commutative algebra of Scheja and Storch. As an application, we compute the Euler classes enriched in bilinear forms associated to arithmetic counts of $d$-planes on complete intersections in $\mathbb{P}^{n}$ in terms of topological Euler numbers over $\mathbb{R}$ and $\mathbb{C}$.
\end{abstract}

Keywords: euler class, A1 homotopy theory, coherent duality, Grassmanians, complete intersections 2020 Mathematics Subject Classification: 14F42, 19E15, 55R40

1 Introduction

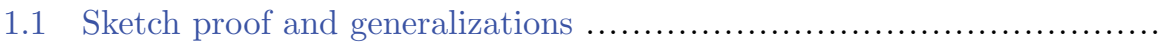

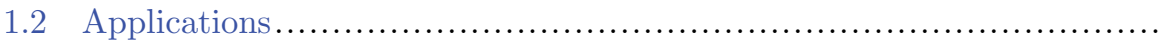

1.3 Notation and conventions .......................................... 686

2 Equality of coherent duality and Poincaré-Hopf Euler numbers $\quad \mathbf{6 8 7}$

2.1 Coherent-duality Euler Number ................................... 687

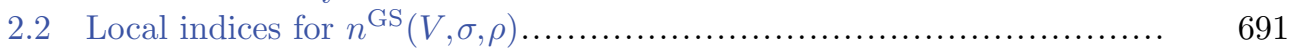

2.3 Scheja-Storch and coherent duality ................................. 695

2.4 The Poincaré-Hopf Euler number with respect to a section.............. 697

$\begin{array}{lll}3 & \text { Cohomology theories for schemes } & \mathbf{7 0 0}\end{array}$

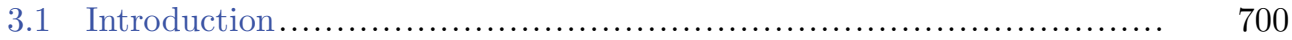

3.2 Features of cohomology theories ................................... 701

3.3 Some cohomology theories ...................................... 702

3.4 The yoga of Euler numbers .................................... 702 
4 Cohomology theories represented by motivic spectra $\quad \mathbf{7 0 5}$

4.1 Aspects of the six-functors formalism ............................... 705

4.2 Cohomology groups and Gysin maps ............................. 707

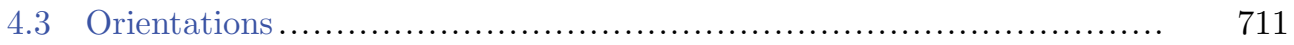

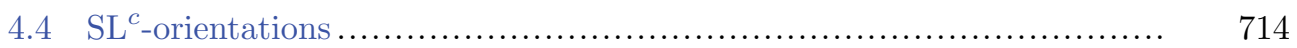

$\begin{array}{llr}5 & \text { Euler classes for representable theories } & \mathbf{7 1 4}\end{array}$

5.1 Tautological Euler class ........................................ 714

5.2 Integrally defined Euler numbers ................................. 715

5.3 Refined Euler classes and numbers............................... 719

5.4 Refined Euler classes and the six-functors formalism ................. $\quad 720$

$6 \quad d$-Dimensional planes on complete intersections in projective space $\mathbf{7 2 2}$

6.1 Some Euler numbers of symmetric powers on Grassmannians ........... 722

6.2 An arithmetic count of the $d$-planes on a complete intersection in projective space ............................................... 731

7 Indices of sections of vector bundles and $\mathbb{A}^{1}$-degrees $\quad 733$

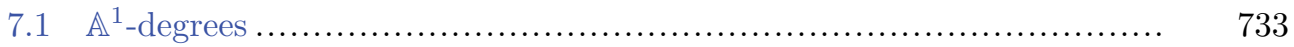

7.2 Main result .................................................. 734

8 Euler numbers in KO-theory and applications $\quad \mathbf{7 3 4}$

$\begin{array}{lll}\text { Appendix A } & \mathrm{KO} \text { via framed correspondences } & \mathbf{7 3 7}\end{array}$

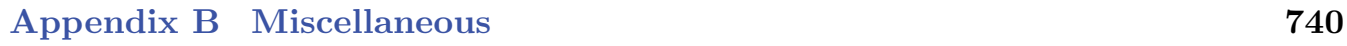

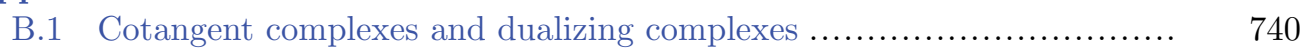

B.2 Grothendieck-Witt rings and Witt rings ........................... 741

B.3 Regular sequences ............................................. 743

$\begin{array}{ll}\text { Acknowledgements } & \mathbf{7 4 3}\end{array}$

$\begin{array}{lr}\text { References } & \mathbf{7 4 3}\end{array}$

\section{Introduction}

For algebraic vector bundles with an appropriate orientation, there are Euler classes and numbers enriched in bilinear forms. We will start over a field $k$ and then discuss more general base schemes, obtaining integrality results. Let $\mathrm{GW}(k)$ denote the GrothendieckWitt group of $k$, defined to be the group completion of the semi-ring of nondegenerate, symmetric, $k$-valued, bilinear forms (see, e.g., [53]). Let $\langle a\rangle$ in $\mathrm{GW}(k)$ denote the class of the rank 1 bilinear form $(x, y) \mapsto a x y$ for $a$ in $k^{*}$.

For a smooth, proper $k$-scheme $f: X \rightarrow \operatorname{Spec} k$ of dimension $n$, coherent duality defines a trace map $\eta_{f}: \mathrm{H}^{n}\left(X, \omega_{X / k}\right) \rightarrow k$, which can be used to construct the following Euler number in $\mathrm{GW}(k)$. Let $V \rightarrow X$ be a rank $n$ vector bundle equipped with a relative orientation, meaning a line bundle $\mathcal{L}$ on $X$ and an isomorphism

$$
\rho: \operatorname{det} V \otimes \omega_{X / k} \rightarrow \mathcal{L}^{\otimes 2}
$$

For $0 \leq i, j \leq n$, let $\beta_{i, j}$ denote the perfect pairing

$$
\beta_{i, j}: \mathrm{H}^{i}\left(X, \wedge^{j} V^{*} \otimes \mathcal{L}\right) \otimes \mathrm{H}^{n-i}\left(X, \wedge^{n-j} V^{*} \otimes \mathcal{L}\right) \rightarrow k
$$


given by the composition

$$
\mathrm{H}^{i}\left(X, \wedge^{j} V^{*} \otimes \mathcal{L}\right) \otimes \mathrm{H}^{n-i}\left(X, \wedge^{n-j} V^{*} \otimes \mathcal{L}\right) \stackrel{\cup}{\rightarrow} \mathrm{H}^{n}\left(X, \wedge^{n} V^{*} \otimes \mathcal{L}^{\otimes 2}\right) \stackrel{\rho}{\rightarrow} \mathrm{H}^{n}\left(X, \omega_{X / k}\right) \stackrel{\eta_{f}}{\rightarrow} k .
$$

For $i=n-i$ and $j=n-j$, note that $\beta_{i, j}$ is a bilinear form on $\mathrm{H}^{i}\left(X, \wedge^{j} V^{*} \otimes \mathcal{L}\right)$. Otherwise, $\beta_{i, j} \oplus \beta_{n-i, n-j}$ determines the bilinear form on $\mathrm{H}^{i}\left(X, \wedge^{j} V^{*} \otimes \mathcal{L}\right) \oplus$ $\mathrm{H}^{n-i}\left(X, \wedge^{n-j} V^{*} \otimes \mathcal{L}\right)$. The alternating sum

$$
n^{\mathrm{GS}}(V):=\sum_{0 \leq i, j \leq n}(-1)^{i+j} \beta_{i, j}
$$

thus determines an element of GW $(k)$, which we will call the Grothendieck-Serre duality or coherent duality Euler number. Note that $\beta_{i, j} \oplus \beta_{n-i, n-j}$ in $\mathrm{GW}(k)$ is an integer multiple of $h$, where $h$ denotes the hyperbolic form $h=\langle 1\rangle+\langle-1\rangle$, with Gram matrix

$$
h=\left[\begin{array}{ll}
0 & 1 \\
1 & 0
\end{array}\right] \text {. }
$$

This notion of the Euler number was suggested by M. J. Hopkins, J.-P. Serre, and A. Raksit, and developed by M. Levine and Raksit for the tangent bundle in [56].

For a relatively oriented vector bundle $V$ equipped with a section $\sigma$ with only isolated zeros, an Euler number $n^{\mathrm{PH}}(V, \sigma)$ was defined in [49, Section 4] as a sum of local indices:

$$
n^{\mathrm{PH}}(V, \sigma)=\sum_{x: \sigma(x)=0} \operatorname{ind}_{x}^{\mathrm{PH}} \sigma .
$$

The index ind ${ }_{x}^{\mathrm{PH}} \sigma$ can be computed explicitly with a formula of Scheja and Storch [67] or of Eisenbud and Levine/Khimshiashvili [29] (see $\$ \S 2.4$ and 2.3) and is also a local degree [48] (this is discussed further in $\S 7$ ). For example, when $x$ is a simple zero of $\sigma$ with $k(x)=k$, the index is given by a well-defined Jacobian Jac $\sigma$ of $\sigma$,

$$
\operatorname{ind}_{x}^{\mathrm{PH}} \sigma=\langle\operatorname{Jac} \sigma(x)\rangle,
$$

illustrating the relation with the Poincaré-Hopf formula for topological vector bundles. (For the definition of the Jacobian, see the beginning of §6.2.) In [49, Section 4, Corollary $36]$, it was shown that $n^{\mathrm{PH}}(V, \sigma)=n^{\mathrm{PH}}\left(V, \sigma^{\prime}\right)$ when $\sigma$ and $\sigma^{\prime}$ are in a family over $\mathbb{A}_{L}^{1}$ of sections with only isolated zeros, where $L$ is a field extension with $[L: k]$ odd. We strengthen this result by equating $n^{\mathrm{PH}}(V, \sigma)$ and $n^{\mathrm{GS}}(V)$; this is the main result of $\S 2$.

Theorem 1.1 (see §2.4). Let $k$ be a field and $V \rightarrow X$ be a relatively oriented, rank $n$ vector bundle on a smooth, proper $k$-scheme of dimension $n$. Suppose $V$ has a section $\sigma$ with only isolated zeros. Then

$$
n^{\mathrm{PH}}(V, \sigma)=n^{\mathrm{GS}}(V) .
$$

In particular, $n^{\mathrm{PH}}(V, \sigma)$ is independent of the choice of $\sigma$.

Remark 1.2 Theorem 1.1 strengthens [15], removing its hypothesis (2) entirely. It also simplifies the proofs of [49, Theorem 1] and [72, Theorems 1 and 2]: it is no longer necessary to show that the sections of certain vector bundles with nonisolated isolated 
zeros are of codimension 2, as in [49, Lemmas 54, 56, and 57] and in [72, Lemma 1], because $n^{\mathrm{PH}}(V, \sigma)$ is independent of $\sigma$.

\subsection{Sketch proof and generalizations}

The proof of Theorem 1.1 proceeds in three steps:

(0) For a section $\sigma$ of $V$, we define an Euler number relative to the section using coherent duality and denote it by $n^{\mathrm{GS}}(V, \sigma, \rho)$. If $\sigma=0$, we recover the absolute Euler number $n^{\mathrm{GS}}(V, \rho)$, essentially by construction.

(1) For two sections $\sigma_{1}, \sigma_{2}$, we show that $n^{\mathrm{GS}}\left(V, \sigma_{1}, \rho\right)=n^{\mathrm{GS}}\left(V, \sigma_{2}, \rho\right)$. To prove this, one can use homotopy invariance of Hermitian $K$-theory or show that $n^{\mathrm{GS}}\left(V, \sigma_{1}, \rho\right)=$ $n^{\mathrm{GS}}(V, \rho)$ by showing an instance of the principle that alternating sums, like Euler characteristics, are unchanged by passing to the homology of a complex.

(2) If a section $\sigma$ has isolated zeros, then $n^{\mathrm{GS}}(V, \sigma, \rho)$ can be expressed as a sum of local indices $\operatorname{ind}_{Z / S}(\sigma)$, where $Z$ is (a clopen component of) the zero scheme of $\sigma$.

(3) For $Z$ a local complete intersection in affine space-that is, in the presence of coordinates - we compute the local degree explicitly and identify it with the SchejaStorch form [2, 67].

Taken together, these steps show that $n^{\mathrm{GS}}(V, \rho)$ is a sum of local contributions given by Scheja-Storch forms, which is essentially the definition of $n^{\mathrm{PH}}(V, \rho)$.

These arguments can be generalized considerably, replacing the Grothendieck-Witt group GW by a more general cohomology theory $E$. We need $E$ to admit transfers along proper lci morphisms of schemes, and an $\mathrm{SL}^{c}$-orientation (see $\S 3$ for more details). Then for step (0) one can define an Euler class $e(V, \sigma, \rho)$ as $z^{*} \sigma_{*}(1)$, where $z$ is the zero section. Step (2) is essentially formal; the main content is in steps (1) and (3). Step (1) becomes formal if we assume that $E$ is $\mathbb{A}^{1}$-invariant. In particular, steps (0)-(2) can be performed for SL-oriented cohomology theories represented by motivic spectra; this is explained in $\S \S 3,4$, and 5 .

It remains to find a replacement for step (3). We offer two possibilities: in $\S 7$ we show that, again in the presence of coordinates, the local indices can be identified with appropriate $\mathbb{A}^{1}$-degrees. On the other hand, in $\S 8$ we show that for $E=\mathrm{KO}$ the motivic spectrum corresponding to Hermitian $K$-theory, the local indices are again given by Scheja-Storch forms. This implies the following:

Corollary 1.3 (see Corollary 8.2 and Definition 3.10). Let $S=\operatorname{Spec}(k)$, where $k$ is $a$ field of characteristic $\neq 2 .^{1}$ Let $\pi: X \rightarrow k$ be smooth and $V / X$ a relatively oriented vector bundle with a nondegenerate section $\sigma$. Write $\varpi: Z=Z(\sigma) \rightarrow k$ for the vanishing scheme (which need not be smooth). Then

$$
n^{\mathrm{PH}}(V, \sigma)=\varpi_{*}(1) \in \mathrm{KO}^{0}(k)=\mathrm{GW}(k) .
$$

\footnotetext{
${ }^{1}$ Here and many times in the text, we restrict to bases $S$ with $1 / 2 \in \mathcal{O}_{S}$ in order for the classical constructions of Hermitian $K$-theory to be well behaved. Forthcoming work by other authors is expected to produce well-behaved Hermitian $K$-theory spectra in all characteristics, and then all our assumptions on the characteristic can be removed.
} 
Here we have used the lci push-forward

$$
\varpi_{*}: \mathrm{KO}^{0}(Z) \stackrel{\rho}{\simeq} \mathrm{KO}^{L_{\varpi}}(Z) \rightarrow \mathrm{KO}^{0}(k)
$$

of Déglise, Jin, and Khan [26]. If, moreover, $X$ is proper, then $\varpi_{*}(1)$ also coincides with $\pi_{*} z^{*} z_{*}(1)$, where $z: X \rightarrow V$ is the zero section (see Corollaries 5.18 and 5.21 and Proposition 5.19). This provides an alternative proof that $n^{\mathrm{PH}}(V, \sigma)$ is independent of the choice of $\sigma$ (under our assumption on $k$ ).

Another important example is when $E$ is taken to be the motivic cohomology theory representing Chow-Witt groups. This recovers the Barge-Morel Euler class $e^{\mathrm{BM}}(V)$ in $\widetilde{\mathrm{CH}}^{r}\left(X, \operatorname{det} V^{*}\right)$ [12], which is defined for a base field of characteristic not 2 . Suppose that $\rho$ is a relative orientation of $V$ and $\pi: X \rightarrow \operatorname{Spec} k$ is the structure map.

Corollary 1.4. Let $k$ be a field of characteristic $\neq 2$. Then $\pi_{*} e^{\mathrm{BM}}(V, \rho)=n^{\mathrm{GS}}(V, \rho)$ in $\mathrm{GW}(k)$.

Proof. We have $e^{\mathrm{BM}}(V, \rho)=e(V, \rho, H \tilde{\mathbb{Z}})$; indeed, by Proposition $5.19, e(V, \rho, H \tilde{\mathbb{Z}})$ can be computed in terms of push-forward along the zero section of $V$, and exactly the same is true for $e^{\mathrm{BM}}$ by definition $[12, \S 2.1]$. We also have $n^{\mathrm{GS}}(V, \rho)=n(V, \rho, \mathrm{KO})$; indeed, the right-hand side is represented by the natural symmetric bilinear form on the cohomology of the Koszul complex by Example 8.1, and this is essentially the definition of $n^{\mathrm{GS}}(V, \rho)$.

It thus suffices to prove that $n(V, \rho, H \tilde{\mathbb{Z}})=n(V, \rho, \mathrm{KO}) \in \mathrm{GW}(k)$. Consider the span of ring spectra $H \tilde{\mathbb{Z}} \leftarrow \tilde{f}_{0} \mathrm{KO} \rightarrow \mathrm{KO}$ as in the proof of Proposition 5.4. It induces an isomorphism on $\pi_{0}(-)(k)$, namely with $\mathrm{GW}(k)$ in all cases. The desired equality follows from the naturality of the Euler numbers.

(An alternative argument proceeds as follows: It suffices to prove that $\pi_{*} e^{\mathrm{BM}}(V, \rho)$ and $n^{\mathrm{GS}}(V, \rho)$ have the same image in $\mathrm{W}(k)$ and $\mathbb{Z}$. The image of $n^{\mathrm{GS}}(V, \rho)$ in $\mathrm{W}(k)$ is given by $n(V, \rho, \mathrm{KW})$; for this we need only show that $e(V, \rho, \mathrm{KW})$ is represented by the Koszul complex, which is Example 5.20. It will thus be enough to show that $n(V, \rho, H \mathbb{Z})=$ $n(V, \rho, \mathrm{KGL})$ and $n\left(V, \rho, \underline{W}\left[\eta^{ \pm}\right]\right)=n(V, \rho, \mathrm{KW})$; this follows as before by considering the spans $H \mathbb{Z} \leftarrow \mathrm{kgl} \rightarrow \mathrm{KGL}$ and $\left.\underline{W}\left[\eta^{ \pm}\right] \leftarrow \mathrm{KW}_{\geq 0} \rightarrow \mathrm{KW}.\right)^{2}$

The left-hand side is the Euler class studied by M. Levine in [55]. We do not compare these Euler classes with the obstruction-theoretic Euler class of [61, Chapter 8]. Asok and Fasel show that the latter agrees with $\pi_{*} e^{\mathrm{BM}}(V, \rho)$ up to a unit in $\mathrm{GW}(k)$ [3].

\subsection{Applications}

It is straightforward to see that Euler numbers for cohomology theories are stable under base change (see Corollary 5.3). This implies that in considering vector bundles on varieties which are already defined over, for example, $\operatorname{Spec}(\mathbb{Z}[1 / 2])$, the possible Euler numbers are constrained to live in $\mathrm{GW}(\mathbb{Z}[1 / 2])=\mathbb{Z}[\langle-1\rangle,\langle 2\rangle] \subset \mathrm{GW}(\mathbb{Q})$. Using novel results on Hermitian $K$-theory [18] allows one to use the base scheme Spec $\mathbb{Z}$ as well.

\footnotetext{
${ }^{2}$ We include this alternative argument because we feel that Example 5.20 is more fully justified in this paper than Example 8.1.
} 
Proposition 5.4 contains both of these cases, and the $\mathbb{Z}[1 / 2]$ case is independent of [18]. It follows that for relatively oriented bundles over $\mathbb{Z}$ the Euler numbers can be read off from topological computations (Proposition 5.9). Over $\mathbb{Z}[1 / 2]$ the topological Euler numbers of the associated real and complex vector bundles, together with one further algebraic computation over some field in which 2 is not a square, determine the Euler number (and this is again independent of [18]); see Theorem 5.11.

We use this to compute a weighted count of $d$-dimensional hyperplanes in a general complete intersection

$$
\left\{f_{1}=\cdots=f_{j}\right\} \hookrightarrow \mathbb{P}_{k}^{n}
$$

over a field $k$. This count depends only on the degrees of the polynomials $f_{i}$ and not on the $f_{i}$ themselves: it is determined by associated real and complex counts, for any $d$ and degrees such that the expected variety of $d$-planes is 0 -dimensional and the associated real count is defined. This is Corollary 6.9. For example, combining with results of Finashin and Kharlamov over $\mathbb{R}[34]$, we have that $160,839\langle 1\rangle+160,650\langle-1\rangle$ and

$$
\begin{aligned}
& 32,063,862,647,475,902,965,720,976,420,325\langle 1\rangle \\
& \quad+32,063,862,647,475,902,965,683,320,692,800\langle-1\rangle
\end{aligned}
$$

are arithmetic counts of the 3-planes in a 7-dimensional cubic hypersurface and in a 16dimensional degree 5 hypersurface, respectively (see Example 6.13). This builds on results of Finashin and Kharlamov [34], J. L. Kass and the second author of the present paper [49], M. Levine [54], S. McKean [58], Okonek and Teleman [62], S. Pauli [66], J. Solomon [70], P. Srinivasan and the second author [72], and M. Wendt [74].

\subsection{Notation and conventions}

1.3.1. Grothendieck duality. We believe that if $f: X \rightarrow Y$ is a morphism of schemes which is locally of finite presentation, then there is a well-behaved adjunction

$$
f_{!}: D_{\mathrm{qcoh}}(X) \leftrightarrows D_{\mathrm{qcoh}}(Y): f^{!}
$$

between the associated derived $\left(\infty\right.$-) categories of unbounded complexes of $\mathcal{O}_{X}$-modules with quasi-coherent homology sheaves. Unfortunately, we are not aware of any references in this generality. Instead, whenever mentioning a functor $f^{!}$, we implicitly assume that $X$ and $Y$ are separated and of finite type over some Noetherian scheme $S$. In this situation, the functor $f^{!}$is constructed for homologically bounded-above complexes in [73, Tag 0A9Y] (see also [24, 40]), and this is all we will use.

1.3.2. Vector bundles. We identify locally free sheaves and vector bundles covariantly, via the assignment

$$
\mathcal{E} \leftrightarrow \operatorname{Spec}\left(\operatorname{Sym}\left(\mathcal{E}^{*}\right)\right)
$$

While it can be convenient to (not) pass to duals here (as in, e.g., [26]), we do not do this, because it confuses the first author terribly. 
1.3.3. Regular sequences and immersions. Following, for example, [14], by a regular immersion of schemes we mean what is called a Koszul-regular immersion in [73, Tag 0638]-that is, a morphism which is locally a closed immersion cut out by a Koszul-regular sequence. Moreover, by a regular sequence we will always mean a Koszuregular sequence [73, Tag 062D], and we reserve the term strongly regular sequence for the usual notion. A strongly regular sequence is regular [73, Tag 062F], whence a strongly regular immersion is regular. In locally Noetherian situations, regular immersions are strongly regular [73, Tags $063 \mathrm{~L}]$.

1.3.4. Cotangent complexes. For a morphism $f: X \rightarrow Y$, we write $L_{f}$ for the cotangent complex. Recall that if $f$ is smooth, then $L_{f} \simeq \Omega_{f}$, whereas if $f$ is a regular immersion, then $L_{f} \simeq C_{f}[1]$, where $C_{f}$ denotes the conormal bundle.

1.3.5. Graded determinants. We write $\widetilde{\operatorname{det}}: K(X) \rightarrow \operatorname{Pic}(D(X))$ for the determinant morphism from Thomason-Trobaugh $K$-theory to the groupoid of graded line bundles. If $C$ is a perfect complex, then we write $\widetilde{\operatorname{det} C}$ for the determinant of the associated $K$-theory point. We write $\operatorname{det} C \in \operatorname{Pic}(X)$ for the ungraded determinant.

Given an lci morphism $f$, we set $\omega_{f}=\operatorname{det} L_{f}$ and $\widetilde{\omega}_{f}=\widetilde{\operatorname{det}} L_{f}$.

We systematically use graded determinants throughout the article; for example, we have the following compact definition of a relative orientation:

Definition 1.5. Let $\pi: X \rightarrow S$ be an lci morphism and $V$ a vector bundle on $X$. By a relative orientation of $V / X / S$ we mean a choice of line bundle $\mathcal{L}$ on $X$ and an isomorphism

$$
\rho: \underline{\operatorname{Hom}}\left(\widetilde{\operatorname{det}} V^{*}, \widetilde{\omega}_{X / S}\right) \stackrel{\simeq}{\rightarrow} \mathcal{L}^{\otimes 2} .
$$

Note that if $\pi$ is smooth, this just means that the locally constant functions $x \mapsto$ $\operatorname{rank}\left(V_{x}\right)$ and $x \mapsto \operatorname{dim} \pi^{-1}(\pi(x))$ on $X$ agree, and that we are given an isomorphism $\mathcal{L}^{\otimes 2} \simeq \omega_{X / S} \otimes \operatorname{det} V$. Hence we recover the definition from [49, Definition 17].

\section{Equality of coherent duality and Poincaré-Hopf Euler numbers}

We prove Theorem 1.1 in this section.

\subsection{Coherent-duality Euler Number}

Let $f: X \rightarrow$ Spec $k$ be a smooth, proper $k$-scheme of dimension $n$, and let $V$ be a rank $n$ vector bundle, relatively oriented by the line bundle $\mathcal{L}$ on $X$ and the isomorphism $\rho: \operatorname{det} V \otimes \omega_{X / k} \rightarrow \mathcal{L}^{\otimes 2}$. Let $\sigma: X \rightarrow V$ be a section, and let $K(\sigma)^{\bullet}$ denote the Koszul complex

$$
0 \rightarrow \wedge^{n} V^{*} \rightarrow \wedge^{n-1} V^{*} \rightarrow \cdots \rightarrow V^{*} \rightarrow \mathcal{O} \rightarrow 0,
$$

with $\mathcal{O}$ in degree 0 and differential of degree +1 given by

$$
d\left(v_{1} \wedge v_{2} \wedge \cdots \wedge v_{j}\right)=\sum_{i=1}^{j}(-1)^{i-1} v_{i}(\sigma) v_{1} \wedge \cdots \wedge v_{i-1} \wedge v_{i+1} \wedge \cdots \wedge v_{j}
$$


This choice of $K(\sigma)^{\bullet}$ is $\operatorname{Hom}_{\mathcal{O}}(-, \mathcal{O})$ applied to the Koszul complex of [27, 17.2]. $K(\sigma)^{\bullet}$ carries a canonical multiplication

$$
m: K(\sigma)^{\bullet} \otimes K(\sigma)^{\bullet} \rightarrow K(\sigma)^{\bullet}
$$

defined in degree $-p$ by $m=\oplus_{i+j=p} 1_{\wedge^{i} V^{*}} \wedge 1_{\wedge^{j} V^{*}}$. Composing $m$ with the projection $p: K(\sigma)^{\bullet} \rightarrow \operatorname{det} V^{*}[n]$ defines a nondegenerate bilinear form

$$
\begin{gathered}
\beta_{(V, \sigma)}: K(V, \sigma) \otimes K(V, \sigma) \rightarrow \operatorname{det} V^{*}[n], \\
\beta_{(V, \sigma)}=p m .
\end{gathered}
$$

Tensoring $\beta_{(V, \sigma)}$ by $\mathcal{L}^{\otimes 2}$ and reordering the tensor factors of the domain, we obtain a nondegenerate symmetric bilinear form on $K(V, \sigma) \otimes \mathcal{L}$ valued in $\left(\operatorname{det} V^{*} \otimes \mathcal{L}^{\otimes 2}\right)[n]$. The orientation $\rho$ determines an isomorphism $\left(\operatorname{det} V^{*} \otimes \mathcal{L}^{\otimes 2}\right)[n] \rightarrow \omega_{X / k}[n]$. Composing $\beta_{(V, \sigma)} \otimes \mathcal{L}^{\otimes 2}$ with this isomorphism produces a nondegenerate bilinear form

$$
\beta_{(V, \sigma, \rho)}:(K(V, \sigma) \otimes \mathcal{L}) \otimes(K(V, \sigma) \otimes \mathcal{L}) \rightarrow \omega_{X / k}[n] .
$$

Let $D(X)$ denote the derived category of quasi-coherent $\mathcal{O}_{X}$-modules. Serre duality determines an isomorphism $R f_{*} \omega_{X / k}[n] \cong \mathcal{O}_{k}$ [41, III Corollary 7.2 and Theorem 7.6]. Since $R f_{*}$ is lax symmetric monoidal (being right adjoint to a symmetric monoidal functor), we obtain a symmetric morphism

$$
R f_{*} \beta_{(V, \sigma, \rho)}:\left[R f_{*}(K(V, \sigma) \otimes \mathcal{L})\right]^{\otimes 2} \rightarrow R f_{*} \omega_{X / k}[n] \simeq \mathcal{O}_{k}
$$

in $D(k)$, which is nondegenerate by Serre duality.

The derived category $D(k)$ is equivalent to the category of graded $k$-vector spaces, by taking cohomology. ${ }^{3}$ If $V$ is a (nondegenerate) symmetric bilinear form in graded $k$-vector spaces, denote by $V^{(n)}=V_{n} \oplus V_{-n}$ (for $n \neq 0$ ) and $V^{(0)}=V_{0}$ the indicated subspaces; observe that they also carry (nondegenerate) symmetric bilinear forms.

Definition 2.1. For a relatively oriented rank $n$ vector bundle $V \rightarrow X$ with section $\sigma$ and orientation $\rho$, over a smooth and proper variety $f: X \rightarrow k$ of dimension $n$, the Grothendieck-Serre-duality Euler number with respect to $\sigma$ is

$$
n^{\mathrm{GS}}(V, \sigma, \rho)=\sum_{i \geq 0}(-1)^{i}\left[\left(R f_{*} \beta_{(V, \sigma, \rho)}\right)^{(i)}\right] \in \mathrm{GW}(k) .
$$

Remark 2.2. In order not to clutter notation unnecessarily, we also write Definition 2.1 as

$$
n^{\mathrm{GS}}(V, \sigma, \rho)=\sum_{i}(-1)^{i}\left[\left(R f_{*} \beta_{(V, \sigma, \rho)}\right)_{i}\right] .
$$

We shall commit to this kind of abuse of notation from now on.

\footnotetext{
${ }^{3}$ In this section, we treat all categories as 1-categories - that is, we ignore the higher structure of $D(k)$ as an $\infty$-category.
} 
Recall that $n^{\mathrm{GS}}(V, \rho) \in \mathrm{GW}(k)$ was defined in the introduction in terms of the symmetric bilinear form on $\bigoplus_{i, j} H^{i}\left(X, \Lambda^{j} V^{*} \otimes \mathcal{L}\right)$.

Proposition 2.3. For any section $\sigma$, we have $n^{\mathrm{GS}}(V, \sigma, \rho)=n^{\mathrm{GS}}(V, \rho) \in \mathrm{GW}(k)$.

To prove Proposition 2.3, we use the hypercohomology spectral sequence $E_{r}^{i, j}\left(K^{\bullet}\right)$ associated to a complex $K^{\bullet}$ of locally free sheaves on $X$ :

$$
E_{1}^{i, j}\left(K^{\bullet}\right):=\mathrm{H}^{j}\left(X, K^{i}\right) \Rightarrow R^{i+j} f_{*} K^{\bullet} .
$$

Let $F_{i}$ denote the resulting filtration on $R^{*} f_{*} K^{\bullet}$, such that

$$
\cdots \supseteq F_{i}=\operatorname{Im}\left(\mathrm{H}^{*}\left(X, K^{\bullet \geq i}\right) \rightarrow \mathrm{H}^{*}\left(X, K^{\bullet}\right)\right) \supseteq F_{i+1} \supseteq \cdots .
$$

Given a perfect symmetric pairing of chain complexes $\beta: K^{\bullet} \otimes K^{\bullet} \rightarrow \omega_{X / k}[n]$, the cup product induces pairings

$$
\beta^{\prime}: R^{*} f_{*} K^{\bullet} \otimes R^{*} f_{*} K^{\bullet} \rightarrow R^{*} f_{*} \omega_{X / k}[n] \rightarrow k
$$

and

$$
\beta_{1}: E_{1}^{*, *}\left(K^{\bullet}\right) \otimes E_{1}^{*, *}\left(K^{\bullet}\right) \rightarrow k .
$$

The following properties hold:

(1) Placing the $k$ in the codomain of $\beta_{1}$ in bidegree $(-n, n), \beta_{1}$ is a map of bigraded vector spaces and satisfies the Leibniz rule with respect to $d_{1}$. It thus induces $\beta_{2}: E_{2}^{*, *}\left(K^{\bullet}\right) \otimes E_{2}^{*, *}\left(K^{\bullet}\right) \rightarrow k$. Then $\beta_{2}$ satisfies the Leibnitz rule with respect to $d_{2}$ and hence induces $\beta_{3}$, and so on.

(2) All the pairings $\beta_{i}$ are perfect.

(3) The pairing $\beta^{\prime}$ is compatible with the filtration in the sense that $\beta^{\prime}\left(F_{i}, F_{k}\right)=0$ if $i+k>-n$.

(4) It follows that $\beta^{\prime}$ induces a pairing on gr $R^{*} f_{*} K^{\bullet}$. Under the isomorphism gr $\simeq E_{\infty}$, it coincides with $\beta_{\infty}$.

(5) $\beta^{\prime}$ is perfect in the filtered sense: the induced pairing $F_{i} \otimes R^{*} f_{*} K^{\bullet} / F_{-n-i+1} \rightarrow k$ is perfect. (In particular, the pairing $\beta^{\prime}$ is perfect.)

Remark 2.4. We do not know a reference for these facts, and proving them would take us too far afield. The main idea is that we have a sequence of duality-preserving functors

$$
C^{\text {perf }}(X) \stackrel{\sigma_{\bullet}}{\longrightarrow} D(X)^{\text {fil }} \stackrel{\pi_{*}}{\longrightarrow} D(k)^{\text {fil }} .
$$

Here $C^{\text {perf }}(X)$ denotes the category of bounded chain complexes of vector bundles, $D(X)^{\text {fil }}$ is the filtered derived category [39], and $\sigma_{\bullet}$ is the 'stupid truncation' functor (composed with forgetting to the filtered derived category). The first duality is with respect to $\underline{\operatorname{Hom}}(-, \omega[n])$, the second with respect to $\underline{\operatorname{Hom}}\left(-, \sigma_{\bullet}(\omega[n])\right)=\underline{\operatorname{Hom}}(-, \omega[n](-n))$, and the third with respect to $\underline{\operatorname{Hom}}(-, k[0](-n))$. There are further duality-preserving functors

$$
(-)^{\mathrm{gr}}: D(k)^{\mathrm{fil}} \rightarrow D(k)^{\mathrm{gr}} \quad \text { and } \quad U: D(k)^{\mathrm{fil}} \rightarrow D(k),
$$


where $D(X)^{\mathrm{gr}}=\operatorname{Fun}(\mathbb{Z}, D(X))$, with $\mathbb{Z}$ viewed as a discrete category. Hence any perfect pairing $C \otimes C \rightarrow k[0](-n) \in D(k)^{\mathrm{fil}}$ induces a perfect pairing on $H_{*} C^{\mathrm{gr}} \otimes H_{*} C^{\mathrm{gr}} \rightarrow$ $k(-n, n)$, satisfying property (1), and a pairing $H_{*} U C \otimes H_{*} U C \rightarrow k$, satisfying properties (3) and (5). Moreover there is a spectral sequence $E_{1}=H_{*} C^{\mathrm{gr}} \Rightarrow H_{*} U C$, satisfying properties (1) and (4). Property (2) is obtained from the fact that passage to homology is a duality-preserving functor.

We apply this to $K^{\bullet} \in C^{\text {perf }}(X)$; then $\operatorname{gr}_{i} \sigma_{\bullet} K^{\bullet}=K^{i}[i]$ and hence $\operatorname{gr}_{i}\left(\pi_{*} \sigma_{\bullet} K^{\bullet}\right)=$ $\pi_{*} K^{i}[i]$.

Lemma 2.5. Let $X$ be a graded $k$-vector space with a finite decreasing filtration

$$
X \supset \cdots \supset X_{\bullet} \supset X_{\bullet+1} \supset \cdots .
$$

Suppose that $X \otimes X \rightarrow k$ is a perfect symmetric bilinear pairing, which is compatible with the filtration in the sense of properties (3) and (5). Let $X^{i}$ denote the ith graded subspace of $X$ and $X_{\bullet}^{i}$ denote the ith graded subspace of $X_{\bullet}$. Then in $\mathrm{GW}(k)$, there is an equality

$$
\sum_{i}(-1)^{i}\left[X^{i}\right]=\sum_{i}(-1)^{i}\left[\operatorname{gr}_{\bullet} X^{i}\right] .
$$

Proof. Note that property (5) implies that the pairing gr $X$ is nondegenerate, so the statement makes sense (recall Remark 2.2). On any graded symmetric bilinear form, the degree $i$ and $-i$ parts for $i \neq 0$ assemble into a metabolic space, with Grothendieck-Witt class determined by the rank (see Lemma B.2). It is clear that the ranks on both sides of our equation are the same; hence it suffices to prove the lemma in the case where $X^{i}=0$ for $i \neq 0$. We may thus ignore the gradings.

Let $N$ be maximal with the property that $X_{N} \neq 0$. We have a perfect pairing

$$
X_{N+1} \otimes X / X_{-n-N} \rightarrow k .
$$

Since $X_{N+1}=0$, we deduce that $X_{-n-N}=X$ and hence $X_{j}=X$ for all $j \leq-n-N$. If $-n-N \geq N$, then $X=X_{N}(N)$ and there is nothing to prove; hence assume the opposite.

We have the perfect pairing

$$
X_{N} / X_{N+1} \otimes X_{-n-N} / X_{-n-N+1} \simeq X_{N} \otimes X / X_{-n-N+1} \rightarrow k .
$$

Pick a sequence of subspaces $X \supset X_{-n-N+1}^{\prime} \supset \cdots \supset X_{N-1}^{\prime}$ such that $X_{i}^{\prime} \subset X_{i}$ and the canonical projection $X_{i}^{\prime} \rightarrow X_{i} / X_{N}$ is an isomorphism. Extend the filtration $X^{\prime}$ by 0 on the left and constantly on the right. By construction, $X_{i}^{\mathrm{gr}}=X_{i}^{\mathrm{gr}}$ for $i \neq N,-n-N$, and the pairing on $X^{\prime} \subset X$ is perfect in the filtered sense. By [59, Lemma I.3.1], we have $X=X^{\prime} \oplus\left(X^{\prime}\right)^{\perp}$. By induction on $N$, we have $\left[X^{\prime}\right]=\left[\mathrm{gr}_{\bullet} X^{\prime}\right]$. It thus suffices to show that $\left[\left(X^{\prime}\right)^{\perp}\right]=\left[\operatorname{gr}_{N} X \oplus \operatorname{gr}_{-n-N} X\right]$. This holds because both sides are metabolic of the same rank: $X_{-n-N}$ is an isotropic subspace of half rank on either side (see again Lemma B.2).

Lemma 2.6. Let $E^{\bullet}$ be a chain complex with a nondegenerate, symmetric bilinear form $E^{\bullet} \otimes E^{\bullet} \rightarrow k[0]$. Then

$$
\sum_{i}(-1)^{i}\left[H^{i}(E)\right]=\sum_{i}\left[E^{i}\right] \in \mathrm{GW}(k) .
$$


Proof. Since passing to homology is a duality-preserving functor, the statement makes sense. Both sides have the same rank, so it suffices to prove equality in $\mathrm{W}(k)$ (see Lemma B.2). We have a perfect pairing $C^{i} \otimes C^{-i} \rightarrow k$, and similarly for homology. Both are metabolic unless $i=0$. We can choose a splitting

$$
C^{0}=H \oplus C^{\prime}
$$

where $H \subset \operatorname{ker}\left(C^{0} \rightarrow C^{1}\right)$ maps isomorphically to $H^{0}(C)$. The restriction of the pairing on $C^{0}$ to $H$ is perfect by construction, and hence $C^{0}=H \oplus H^{\perp}$. It suffices to show that $H^{\perp}$ is metabolic. Compatibility of the pairing with the differential shows that $d\left(C^{-1}\right) \subset C^{0}$ is an isotropic subspace. Self-duality shows that

$$
\operatorname{Im}\left(d: C^{-1} \rightarrow C^{0}\right) \simeq \operatorname{Im}\left(d^{\vee}:\left(C^{1}\right)^{\vee} \rightarrow\left(C^{0}\right)^{\vee}\right) \simeq \operatorname{Im}\left(d: C^{0} \rightarrow C^{1}\right)^{\vee},
$$

which implies that $d\left(C^{-1}\right) \subset H^{\perp}$ is of half rank. This concludes the proof.

Proof of Proposition 2.3. Let $K^{\bullet}=K(V, \sigma)^{\bullet} \otimes \mathcal{L}$. We compute

$$
\begin{aligned}
& n^{\mathrm{GS}}(V, \rho) \stackrel{\text { def. }}{=} \sum(-1)^{i+j}\left[E_{1}^{i, j}\left(K^{\bullet}\right)\right] \\
& \stackrel{\text { Lemma }}{=}{ }^{2.6} \sum(-1)^{i+j}\left[E_{\infty}^{i, j}\left(K^{\bullet}\right)\right] \\
& \stackrel{\text { Lemma }}{=} \sum_{i}(-1)^{i}\left[R^{i} f_{*} K^{\bullet}\right] \\
& \stackrel{\text { def. }}{=} n^{\mathrm{GS}}(V, \sigma, \rho) .
\end{aligned}
$$

This is the desired result.

Remark 2.7. Admitting a version of Hermitian $K$-theory which is $\mathbb{A}^{1}$-invariant on regular schemes and has proper push-forwards, one can give an alternative proof of Proposition 2.3 by considering the Koszul complex with respect to the section $t \sigma$ on $\mathbb{A}^{1} \times X$. While we believe such a theory exists, at the time of writing there is no reference for this in characteristic 2 , so we chose to present our argument instead.

\subsection{Local indices for $n^{\mathrm{GS}}(V, \sigma, \rho)$}

Suppose that $\sigma$ is a section with only isolated zeros. Let $i$ denote the closed immersion $i: Z=Z(\sigma) \hookrightarrow X$ given by the zero locus of $\sigma$. We express $n^{\mathrm{GS}}(V, \sigma, \rho)$ as a sum over the points $z$ of $Z$ of a local index at $z$. To do this, we use a push-forward in a suitable context and show that $\beta_{(V, \sigma)}$ is a push-forward from $Z$.

For a line bundle $\mathcal{L}$ on a scheme $X$, denote by $\mathrm{BL}_{\text {naive }}(D(X), \mathcal{L}[n])$ the set of isomorphism classes of nondegenerate symmetric bilinear forms on the derived category of perfect complexes on $X$, with respect to the duality $\underline{\operatorname{Hom}}(-, \mathcal{L}[n])$. For a proper, lci map $f: X^{\prime} \rightarrow$ $X$, coherent duality supplies us with a trace map $\eta_{f, \mathcal{L}}: f_{*} f^{!}(\mathcal{L}) \rightarrow \mathcal{L}$. We can use this $[21$, Theorem 4.2.9] to build a push-forward 


$$
\begin{gathered}
f_{*}: \mathrm{BL}_{\text {naive }}\left(D\left(X^{\prime}\right), f^{!} \mathcal{L}\right) \rightarrow \mathrm{BL}_{\text {naive }}(D(X), \mathcal{L}), \\
{\left[E \otimes E \stackrel{\phi}{\rightarrow} f^{!} \mathcal{L}\right] \mapsto\left[f_{*} E \otimes f_{*} E \rightarrow f_{*}(E \otimes E) \stackrel{f_{*} \phi}{\longrightarrow} f_{*}\left(f^{!} \mathcal{L}\right) \stackrel{\eta_{f, \mathcal{L}}}{\longrightarrow} \mathcal{L}\right] .}
\end{gathered}
$$

Remark 2.8. There is a canonical weak equivalence $f^{!} \mathcal{L} \simeq f^{!} \mathcal{O}_{X} \otimes f^{*} \mathcal{L}$, and $\eta_{f, \mathcal{L}}$ is given by the composition

$$
f_{*}\left(f^{!} \mathcal{L}\right) \simeq f_{*}\left(f^{!} \mathcal{O}_{X} \otimes f^{*} \mathcal{L}\right) \simeq f_{*} f^{!} \mathcal{O}_{X} \otimes \mathcal{L} \stackrel{\eta_{f} \otimes \mathrm{id}_{\mathcal{L}}}{\longrightarrow} \mathcal{L}
$$

where $\eta_{f}=\eta_{f, \mathcal{O}_{S}}[73$, Lemma 47.17.8].

Example 2.9. Consider the case of a relatively oriented vector bundle $V$ on a smooth, proper variety $f: X \rightarrow \operatorname{Spec}(k)$. Note that elements of $\mathrm{BL}^{\text {naive }}(k)$ are just isomorphism classes of symmetric bilinear forms on graded vector spaces. The orientation supplies us with an equivalence

$$
f^{!}\left(\mathcal{O}_{k}\right) \simeq \omega_{X / k}[n] \simeq \operatorname{det} V^{*}[n] \otimes \mathcal{L}^{\otimes 2} .
$$

Under the induced push-forward map we have

$$
f_{*}\left[\beta_{(V, \sigma, \rho)}\right]=n^{\mathrm{GS}}(V, \sigma, \rho) \in \mathrm{BL}^{\text {naive }}(k),
$$

where $\beta_{(V, \sigma, \rho)} \in \mathrm{BL}^{\text {naive }}\left(X, \operatorname{det} V^{*}[n] \otimes \mathcal{L}^{\otimes 2}\right)$ is the form on $K(V, \sigma) \otimes \mathcal{L}$ defined in $\S 2.1$.

Remark 2.10. A symmetric bilinear form $\phi$ on the derived category $D(S)$ is usually not a very sensible notion. We offer three ways around this:

(1) If $1 / 2 \in S$, we could look at the image of $\phi$ in the Balmer-Witt group of $S$.

(2) If $\phi$ happens to be concentrated in degree 0 , it corresponds to a symmetric bilinear form on a vector bundle on $S$, which is a sensible invariant.

(3) If $S=\operatorname{Spec}(k)$ is the spectrum of a field, then $D(S)$ is equivalent to the category of graded vector spaces, and we can split $\phi$ into components by degree and consider

$$
c l(\phi):=\left[H^{0}(\phi)\right]+\sum_{i>0}(-1)^{i}\left[H^{i}(\phi) \oplus H^{-i}(\phi)\right] \in \mathrm{GW}(k) .
$$

Let $1_{Z}$ denote the element of $\mathrm{BL}_{\text {naive }}\left(D(Z), \mathcal{O}_{Z}[0]\right)$ represented by $\mathcal{O}_{Z} \otimes \mathcal{O}_{Z} \rightarrow \mathcal{O}_{Z}$.

Proposition 2.11. Let $X$ be a scheme, $V$ a vector bundle, and $\sigma \in \Gamma(X, V)$ a section locally given by a regular sequence. Write $i: Z=Z(\sigma) \hookrightarrow X$ for the inclusion of the zero scheme. Proposition B.1 yields a canonical equivalence $i^{!} \operatorname{det}\left(V^{*}\right)[n] \simeq \mathcal{O}_{Z}[0]$, where $n$ is the rank of $V$; under the induced map

$$
i_{*}: \mathrm{BL}_{\text {naive }}\left(D(Z), \mathcal{O}_{Z}[0]\right) \rightarrow \mathrm{BL}_{\text {naive }}\left(D(X), \operatorname{det}\left(V^{*}\right)[n]\right),
$$

we have $i_{*}\left(1_{Z}\right)=\beta_{(V, \sigma)}$, where

$$
\beta_{(V, \sigma)}: K(V, \sigma) \otimes K(V, \sigma) \rightarrow \operatorname{det}\left(V^{*}\right)[n]
$$

is the canonical pairing on the Koszul complex as in §2.1. 
Proof. Because $\sigma$ locally corresponds to a regular sequence, the canonical map $r$ : $K(V, \sigma)^{\bullet} \rightarrow i_{*} \mathcal{O}_{Z}$ is an equivalence in $D(X)$. The canonical projection $i_{*} \mathcal{O}_{Z} \simeq K(V, \sigma) \rightarrow$ $\operatorname{det}\left(V^{*}\right)[n]$ induces by adjunction a map $\mathcal{O}_{Z} \rightarrow i^{!} \operatorname{det}\left(V^{*}\right)[n]$. We claim that this is the equivalence of Proposition B.1. The proof of that proposition shows that the problem is local on $Z$, so we may assume that $V$ is trivial. Then this map is precisely the isomorphism constructed in [40, Proposition III.7.2 and preceeding pages], which is also the isomorphism used in the proof of Proposition B.1.

Now we prove that $i_{*}\left(1_{Z}\right)=\beta_{(V, \sigma)}$. Consider the following diagram:

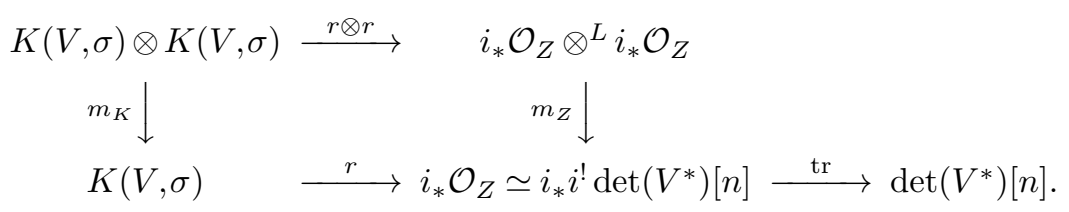

The map $m_{K}: K(V, \sigma) \otimes K(V, \sigma) \rightarrow K(V, \sigma)$ is the canonical multiplication (see $\S 2.1$, property (2)) and $m_{Z}: i_{*} \mathcal{O}_{Z} \otimes{ }^{L} i_{*} \mathcal{O}_{Z} \rightarrow i_{*} \mathcal{O}_{Z} \otimes i_{*} \mathcal{O}_{Z} \rightarrow i_{*} \mathcal{O}_{Z}$ is equivalently given by either multiplication in $\mathcal{O}_{Z}$ or the lax monoidal witness transformation of $i_{*}$. The former interpretation shows that the left-hand square commutes. The pairing $i_{*}(1)$ is given by the composite from the top right corner to the bottom right corner. To prove the claim, it suffices to show that the bottom-row composite $K(V, \sigma) \rightarrow \operatorname{det}\left(V^{*}\right)[n]$ is the canonical projection. This follows by adjunction from our choice of equivalence $\mathcal{O}_{Z} \simeq i^{!} \operatorname{det}\left(V^{*}\right)[n]$.

This concludes the proof.

Proposition 2.11 is an example of a more general phenomenon given in MetaTheorem 3.9.

Lemma 2.12 ([21]). Let $g: Z \rightarrow Y$ and $f: Y \rightarrow X$ be proper maps. ${ }^{4}$ Given equivalences $f^{!} \mathcal{L} \simeq \mathcal{M}[n]$ and $g^{!} \mathcal{M}[n] \simeq \mathcal{N}$, the canonical equivalence $(f g)^{!} \simeq g^{!} f^{!}$produces a weak equivalence $(f g)^{!} \mathcal{L} \simeq \mathcal{N}$, and consequently push-forward maps

$$
\begin{gathered}
g_{*}: \mathrm{BL}_{\text {naive }}(D(Z), \mathcal{N}) \rightarrow \mathrm{BL}_{\text {naive }}(D(Y), \mathcal{M}[n]), \\
f_{*}: \mathrm{BL}_{\text {naive }}(D(Y), \mathcal{M}[n]) \rightarrow \mathrm{BL}_{\text {naive }}(D(X), \mathcal{L}), \\
(f g)_{*}: \mathrm{BL}_{\text {naive }}(D(Z), \mathcal{N}) \rightarrow \mathrm{BL}_{\text {naive }}(D(X), \mathcal{L}) .
\end{gathered}
$$

There is a canonical equivalence $(f g)_{*} \simeq f_{*} g_{*}$.

Proof. The main point is that $\eta_{f, \mathcal{L}} \circ f_{*}\left(\eta_{g, \mathcal{M}[n]}\right)=\eta_{f g, \mathcal{L}}$. The categorical details are worked out in the reference.

Now we get back to our Euler numbers. Let $X / k$ be smooth and proper, $V$ a relatively oriented vector bundle, and $\sigma$ a section of $V$ with only isolated zeros. Write $i: Z=Z(\sigma) \hookrightarrow$

\footnotetext{
${ }^{4}$ Recall our convention that since we are invoking a functor $f^{!}$, then $Z, Y, X$ are also of finite type and separated over a Noetherian base $S$. Without this we should add the hypothesis that $f, g$ are locally (so globally) of finite presentation.
} 
$X$ for the inclusion of the zero scheme of $\sigma$. Let $\varpi: Z \rightarrow$ Spec $k$ and $f: X \rightarrow$ Spec $k$ denote the structure maps, so that $\varpi=f i$.

The weak equivalence $i^{!} \operatorname{det}\left(V^{*}\right)[n] \simeq \mathcal{O}_{Z}[0]$ of Proposition 2.11, with Remark 2.8, produces a weak equivalence $i^{!}\left(\operatorname{det} V^{*}[n] \otimes \mathcal{L}^{\otimes 2}\right) \cong i^{*} \mathcal{L}^{\otimes 2}$. The orientation $\rho$ gives an isomorphism $\operatorname{det} V^{*}[n] \otimes \mathcal{L}^{\otimes 2} \cong \omega_{X / k}[n]$. Combining, we have a chosen weak equivalence

$$
i^{!}\left(\omega_{X / k}[n]\right) \cong i^{*} \mathcal{L}^{\otimes 2} .
$$

Since also $f^{!} \mathcal{O}_{k} \simeq \omega_{X / k}[n]$ (see, e.g., Proposition B.1), we therefore obtain a canonical equivalence

$$
\varpi ! \mathcal{O}_{k} \simeq i^{*} \mathcal{L}^{\otimes 2}
$$

We use this equivalence to define

$$
\varpi_{*}: \mathrm{BL}^{\text {naive }}\left(Z, i^{*} \mathcal{L}^{\otimes 2}\right) \rightarrow \mathrm{BL}^{\text {naive }}(k) .
$$

Corollary 2.13. With this notation, we have

$$
n^{\mathrm{GS}}(V, \sigma, \rho)=\varpi_{*}\left(i^{*} \mathcal{L} \otimes i^{*} \mathcal{L} \rightarrow i^{*} \mathcal{L}^{\otimes 2}\right) .
$$

Proof. By Lemma 2.12 we have $\varpi_{*}=f_{*} i_{*}$. Proposition 2.11 and the projection formula imply that $i_{*}\left(i^{*} \mathcal{L} \otimes i^{*} \mathcal{L} \rightarrow i^{*} \mathcal{L}^{\otimes 2}\right)=\beta_{V, \sigma, \rho}$. We conclude by Example 2.9.

Suppose that $\sigma$ has isolated zeros, or in other words that the support of $\sigma$ is a disjoint union of points. Then $n^{\mathrm{GS}}(V, \sigma, \rho)$ can be expressed as a sum of local contributions. Namely, for each point $z$ of $Z$, let $i_{z}: Z_{z} \hookrightarrow X$ denote the chosen immersion coming from the connected component of $Z$ given by $z$. Let $\varpi_{z}: Z_{z} \rightarrow$ Spec $k$ denote the structure map. Then

$$
n^{\mathrm{GS}}(V, \sigma, \rho)=\sum_{z \in Z} \varpi_{z *}\left(i_{z}^{*} \mathcal{L} \otimes i_{z}^{*} \mathcal{L} \rightarrow i_{z}^{*} \mathcal{L}^{\otimes 2}\right) .
$$

In light of this we propose the following:

Definition 2.14. For a relatively oriented vector bundle with a section as described, and $z \in Z(\sigma)$, we define

$$
\operatorname{ind}_{z}(\sigma)=\operatorname{ind}_{z}(V, \sigma, \rho)=\varpi_{z *}\left(i_{z}^{*} \mathcal{L} \otimes i_{z}^{*} \mathcal{L} \rightarrow i_{z}^{*} \mathcal{L}^{\otimes 2}\right) \in \mathrm{BL}^{\text {naive }}(k) .
$$

The previous formula then reads

$$
n^{\mathrm{GS}}(V, \sigma, \rho)=\sum_{z \in Z} \operatorname{ind}_{z}(V, \sigma, \rho) .
$$

In the next two subsections, we compute the local contributions $\operatorname{ind}_{z}(\sigma)$ as an explicit bilinear form constructed by Scheja and Storch [67], appearing in the Eisenbud-LevineKhimshiashvili signature theorem [29] and used as the local index of the Euler class constructed in [49, Section 4]. 


\subsection{Scheja-Storch and coherent duality}

Let $S$ be a scheme, $\pi: X \rightarrow S$ a smooth scheme of relative dimension $n$, and $Z \subset X$ closed with $\varpi: Z \rightarrow S$ finite. Suppose the following data:

(1) sections $T_{1}, \ldots, T_{n} \in \mathcal{O}(X)$ such that $T_{i} \otimes 1-1 \otimes T_{i}$ generate the ideal of $X \subset X \times{ }_{S} X$;

(2) sections $f_{1}, \ldots, f_{n} \in \mathcal{O}(X)$ such that $Z=Z\left(f_{1}, \ldots, f_{n}\right)$.

Remark 2.15. Since $Z \rightarrow X$ is quasi-finite, Lemma B.5 shows that $f_{1}, \ldots, f_{n}$ is a regular sequence and $Z \rightarrow X$ is flat, so finite locally free (being finite and finitely presented [73, Tag 02KB]).

Choose $a_{i j} \in \mathcal{O}\left(X \times_{S} X\right)$ such that

$$
f_{i} \otimes 1-1 \otimes f_{i}=\sum_{j} a_{i j}\left(T_{j} \otimes 1-1 \otimes T_{j}\right) .
$$

Let $\Delta \in \mathcal{O}\left(Z \times{ }_{S} Z\right)$ be the image of the determinant of $a_{i j}$. Since $\varpi$ is finite locally free, $\Delta$ determines an element $\tilde{\Delta}$ of

$\operatorname{Hom}_{\mathcal{O}_{S}}\left(\mathcal{O}_{S},\left(\varpi \times_{S} \varpi\right)_{*} \mathcal{O}_{Z \times_{S} Z}\right) \simeq \operatorname{Hom}_{\mathcal{O}_{S}}\left(\mathcal{O}_{S}, \varpi_{*} \mathcal{O}_{Z} \otimes \varpi_{*} \mathcal{O}_{Z}\right) \simeq \operatorname{Hom}_{\mathcal{O}_{S}}\left(\left(\varpi_{*} \mathcal{O}_{Z}\right)^{*}, \varpi_{*} \mathcal{O}_{Z}\right)$

Remark 2.16. We can make $\tilde{\Delta}$ explicit: if $\Delta=\sum_{i} b_{i} \otimes b_{i}^{\prime}$, then

$$
\tilde{\Delta}(\alpha)=\sum_{i} \alpha\left(b_{i}\right) b_{i}^{\prime} .
$$

Remark 2.17. By construction, the pullback of $\Delta$ along the diagonal $\delta: Z \rightarrow Z \times{ }_{S} Z$ is the determinant of the differentiation map $\left.C_{Z / X} \rightarrow \Omega_{X}\right|_{Z}$ with respect to the canonical bases. In other words, this is the Jacobian:

$$
\delta^{*}(\Delta)=\operatorname{Jac} F:=\operatorname{det}\left(\frac{\partial f_{i}}{\partial T_{j}}\right)_{i, j=1}^{n} .
$$

Theorem 2.18. Under the foregoing assumptions, the map

$$
\tilde{\Delta}:\left(\varpi_{*} \mathcal{O}_{Z}\right)^{*} \rightarrow \varpi_{*} \mathcal{O}_{Z}
$$

is a symmetric isomorphism and hence determines a symmetric bilinear structure on $\varpi_{*} \mathcal{O}_{Z}$. This is the same structure as $\varpi_{*}(1)$-that is,

$$
\varpi_{*}\left(\mathcal{O}_{Z}\right) \otimes \varpi_{*}\left(\mathcal{O}_{Z}\right) \rightarrow \varpi_{*}\left(\mathcal{O}_{Z}\right) \simeq \varpi_{*}\left(\varpi^{!} \mathcal{O}_{S}\right) \stackrel{\eta_{\varpi}}{\longrightarrow} \mathcal{O}_{S}
$$

Here the isomorphism $\varpi^{!} \mathcal{O}_{Z} \simeq \mathcal{O}_{Z}$ arises from

$$
\varpi^{!}\left(\mathcal{O}_{Z}\right) \simeq \widetilde{\operatorname{det}} L_{\varpi} \simeq \omega_{Z / X} \otimes \omega_{X / S} \simeq \mathcal{O},
$$

with the first isomorphism given by Proposition B.1 and the third given by the sections $\left(T_{i}\right)$ and $\left(f_{i}\right)$.

Remark 2.19. The theorem asserts in particular that the isomorphism $\tilde{\Delta}$, and hence the section $\Delta$, is independent of the choice of the $a_{i j}$. 
We begin with some preliminary observations before delving into the proof. The problem is local on $S$, so we may assume that $S=\operatorname{Spec}(A)$; then $Z=\operatorname{Spec}(B)$. Since $\varpi$ is finite, there is a canonical isomorphism [40, III $\S 8$ Theorem 8.7 (3), or Ideal Theorem (3) p. 6]

$$
\varpi^{!} \simeq \underline{\operatorname{Hom}}_{A}(B,-): D(A) \rightarrow D(B) .
$$

In particular,

$$
\varpi !(A) \simeq \underline{\operatorname{Hom}}_{A}(B, A)
$$

and the trace map takes the form [40, Ideal theorem 3) pg 7]

$$
\varpi_{*} \varpi^{!} A \simeq \operatorname{Hom}_{A}(B, A) \rightarrow A, \eta \mapsto \eta(1) .
$$

Proof of Theorem 2.18. The isomorphisms

$$
B^{*}=\underline{\operatorname{Hom}}_{A}(B, A) \simeq \varpi^{!}(A) \simeq B
$$

determine an element $\Delta^{\prime} \in \operatorname{Hom}_{A}\left(B^{*}, B\right)$. The theorem is equivalent to showing that $\tilde{\Delta}=\Delta^{\prime}$.

We thus need to make explicit the isomorphism

$$
\underline{\operatorname{Hom}}_{A}(B, A) \simeq \varpi^{!}\left(\mathcal{O}_{A}\right) \simeq i^{!} \pi^{!}\left(\mathcal{O}_{A}\right) \simeq \omega_{Z / X} \otimes \omega_{X} \simeq \mathcal{O}
$$

Tracing through the definitions (including the proof of [40, III Proposition 8.2]), one finds that this isomorphism arises by computing

$$
\operatorname{Ext}_{X}^{n}\left(B, \mathcal{O}_{X}\right)
$$

in two ways. One the one hand, the kernel of the surjection $\mathcal{O}_{X} \rightarrow B$ is generated by $f_{1}, \ldots, f_{n}$, which is a regular sequence by Remark 2.15 ; let $K_{A}(f)^{\bullet}$ denote the corresponding Koszul complex. On the other hand, we can consider the embedding $Z \hookrightarrow X \times Z$; its ideal is generated by the strongly regular sequence $T_{i}-t_{i}$, where $t_{i}$ is the image of $T_{i}$ in $B$. We thus obtain a resolution $K_{B}(T-t)^{\bullet} \rightarrow B$ over $X \times Z$. Since $p: X \times Z \rightarrow X$ is finite, $p_{*} K_{B}(T-t)^{\bullet} \rightarrow p_{*} B=B$ is still a resolution. We shall conflate $K_{B}(T-t)$ and $p_{*} K_{B}(T-t)$ notationally. We can thus compute

$$
\operatorname{Ext}_{X}^{n}\left(B, \mathcal{O}_{X}\right) \simeq \operatorname{coker}\left(\operatorname{Hom}_{X}\left(K_{B}(T-t)^{n-1}, \mathcal{O}_{X}\right) \rightarrow \operatorname{Hom}_{X}\left(K_{B}(T-t)^{n}, \mathcal{O}_{X}\right)\right) .
$$

Since $\operatorname{Hom}_{X}\left(B \otimes \mathcal{O}_{X}, \mathcal{O}_{X}\right) \simeq \operatorname{Hom}_{A}\left(B, \mathcal{O}_{X}\right) \simeq \operatorname{Hom}_{A}(B, A) \otimes_{A} \mathcal{O}_{X}$, there is a natural map $\xi: \operatorname{Hom}_{A}(B, A) \rightarrow \operatorname{Hom}_{X}\left(K_{B}(T-t)^{n}, \mathcal{O}_{X}\right)$ (sending $\alpha$ to $\left.\alpha \otimes 1\right)$. One checks that this induces $\operatorname{Hom}_{A}(B, A) \simeq \operatorname{coker}(\ldots) \simeq \operatorname{Ext}_{X}^{n}\left(B, \mathcal{O}_{X}\right)$.

We can write down a map of resolutions $\zeta: K_{A}(f) \rightarrow K_{B}(T-t)$ as follows: The kernel of $B \otimes \mathcal{O}_{X} \rightarrow B$ is by construction generated by $\left\{T_{i}-t_{i}\right\}_{i}$, but it also contains $f_{i}$. Note that $f_{i}=\sum_{j} \bar{a}_{i j}\left(T_{j}-t_{j}\right)$, where we write $\bar{a}_{i j}$ for the image of $a_{i j}$ in $\mathcal{O}_{X} \otimes B$. Letting $K_{A}(f)$ be the exterior algebra on $\left\{e_{1}, \ldots, e_{n}\right\}$ and $K_{B}(T-t)$ the exterior algebra on $\left\{e_{1}^{\prime}, \ldots, e_{n}^{\prime}\right\}$, the map $\zeta$ is specified by $\zeta\left(e_{i}\right)=\sum_{j} \bar{a}_{i j} e_{j}^{\prime}$. The isomorphism

$$
\operatorname{Hom}_{A}(B, A) \simeq h^{n} \operatorname{Hom}_{X}\left(K_{B}(T-t)^{\bullet}, \mathcal{O}_{X}\right) \simeq h^{n} \operatorname{Hom}_{X}\left(K_{A}(f)^{\bullet}, \mathcal{O}_{X}\right) \simeq B
$$


is thus given by

$$
\begin{gathered}
\operatorname{Hom}_{A}(B, A) \stackrel{\xi}{\rightarrow} \operatorname{Hom}_{X}\left(K_{B}(T-t)^{n}, \mathcal{O}_{X}\right) \stackrel{\operatorname{det}\left(a_{i j}\right)^{*}}{\longrightarrow} \operatorname{Hom}_{X}\left(K_{A}(f)^{n}, \mathcal{O}_{X}\right) \\
\simeq \operatorname{Hom}_{X}\left(\mathcal{O}_{X}, \mathcal{O}_{X}\right) \simeq \mathcal{O}_{X} \rightarrow B
\end{gathered}
$$

Write the image of $\operatorname{det}\left(a_{i j}\right)$ in $B \otimes B$ as $\sum_{k} b_{k} \otimes b_{k}^{\prime}$. Tracing through the definitions, we find that this composite sends $\alpha \in \operatorname{Hom}_{A}(B, A)$ to $\sum_{k} \alpha\left(b_{k}\right) b_{k}^{\prime}$. By Remark 2.16, this is precisely $\tilde{\Delta}$.

This concludes the proof.

Definition 2.20. If $X=U \subset \mathbb{A}_{S}^{n},\left(T_{i}\right)$ are the standard coordinates, and $F=\left(f_{1}, \ldots, f_{n}\right)$, we denote the symmetric bilinear form already constructed by

$$
\langle-\mid-\rangle^{\mathrm{SS}}=\langle-\mid-\rangle^{\mathrm{SS}}(U, F, S) .
$$

This form was first constructed, without explicitly using coherent duality, by Scheja and Storch [2, 67].

Example 2.21. Suppose that $Z \rightarrow S$ is an isomorphism (where $Z=Z(F)$ as before, so that the diagonal $\delta: Z \rightarrow Z \times{ }_{S} Z$ is also an isomorphism. Then $\langle-\mid-\rangle^{\mathrm{SS}}$ is just the rank 1 bilinear form corresponding to multiplication by $\delta^{*}(\Delta) \in \mathcal{O}_{Z} \simeq \mathcal{O}_{S}$. In other words, using Remark 2.17, $\langle-\mid-\rangle^{\mathrm{SS}}$ identifies with $(x, y) \mapsto(\mathrm{Jac} F) x y$.

\subsection{The Poincaré-Hopf Euler number with respect to a section}

In this subsection, we recall the Euler class defined in [49, Section 4] and prove Theorem 1.1. To distinguish this Euler class from the others under consideration, here we call it the Poincaré-Hopf Euler number, because it is a sum of local indices as in the Poincaré-Hopf theorem for the Euler characteristic of a manifold. It is defined using local coordinates.

Let $k$ be a field, and let $X$ be an $n$-dimensional smooth $k$-scheme. Let $z$ be a closed point of $X$.

Definition 2.22 (compare [49, Definition 17]). By a system of Nisnevich coordinates around $z$ we mean a Zariski open neighborhood $U$ of $z$ in $X$, and an étale map $\varphi: U \rightarrow \mathbb{A}_{k}^{n}$ such that the extension of residue fields $k(\varphi(z)) \subseteq k(z)$ is an isomorphism.

Proposition 2.23. When $n>0$, there exists a system of Nisnevich coordinates around every closed point $z$ of $X$.

Proof. When $k$ is infinite, this follows from [52, Chapter 8. Proposition 3.2.1]. When $k(z) / k$ is separable, for instance when $k$ is finite, this is [49, Lemma 18].

As before, let $V$ be a relatively oriented, rank $n$ vector bundle on $X$. Let $\sigma$ be a section with only isolated zeros, and let $Z \hookrightarrow X$ denote the closed subscheme given by the zero locus of $\sigma$. Let $z$ be a point of $Z$. The Poincaré-Hopf local index or degree

$$
\operatorname{ind}_{z}^{\mathrm{PH}} \sigma \in \mathrm{GW}(k)
$$

was defined in [49, Definition 30] as follows: Choose a system of Nisnevich coordinates $\varphi: U \rightarrow \mathbb{A}_{k}^{n}$ around $z$. After possibly shrinking $U$, the restriction of $V$ to $U$ is trivial 
and we may choose an isomorphism $\psi:\left.V\right|_{U} \rightarrow \mathcal{O}_{U}^{n}$ of $V$. The local trivialization $\psi$ induces a distinguished section of $\operatorname{det} V(U)$. The system of local coordinates $\varphi$ induces a distinguished section of $\operatorname{det} T_{X}(U)$, and we therefore have a distinguished section of $\left(\operatorname{det} V \otimes \omega_{X}\right)(U)$. As in [49, Definition 19], the local coordinates $\varpi$ and local trivialization $\psi$ are said to be compatible with the relative orientation if the distinguished element is the tensor square of a section of $\mathcal{L}(U)$. By multiplying $\psi$ by a section in $\mathcal{O}(U)$, we may assume this compatibility.

Under $\psi$, the section $\sigma$ can be identified with an $n$-tuple $\left(f_{1}, \ldots, f_{n}\right)$ of regular functions, $\psi(\sigma)=\left(f_{1}, \ldots, f_{n}\right) \in \oplus_{i=1}^{n} \mathcal{O}_{U}$. Let $m$ denote the maximal ideal of $\mathcal{O}_{U}$ corresponding to $z$. Since $z$ is an isolated zero, there is an integer $n$ such that $m^{n}=0$ in $\mathcal{O}_{Z, z}$. For any $N$, it is possible to choose $\left(g_{1}, \ldots, g_{n}\right)$ in $\oplus_{i=1}^{n} m^{N}$ such that $\left.\left(f_{i}+g_{i}\right)\right|_{U}$ is in the image of $\varphi^{*}: \mathcal{O}_{\mathbb{A}_{S}^{r}} \rightarrow \varphi_{*} \mathcal{O}_{U}$ after possibly shrinking $U$ [49, Lemma 22]. For $N=2 n$, choose such $\left(g_{1}, \ldots, g_{n}\right)$ and let $F_{i}$ in $\mathcal{O}_{\mathbb{A}_{k}^{d}}\left(\mathbb{A}_{k}^{d}\right)$ be the functions such that $\varphi^{*}\left(F_{i}\right)=f_{i}+g_{i}$. Then $\varphi$ induces an isomorphism $\mathcal{O}_{Z, z} \cong k\left[t_{1}, \ldots, t_{n}\right]_{m_{\varphi(z)}} /\left(F_{1}, \ldots, F_{n}\right)[49$, Lemma 25$]$, and ind ${ }_{z}^{\mathrm{PH}} \sigma$ is defined to be the associated Scheja-Storch form $\langle-\mid-\rangle^{\mathrm{SS}}(\varphi(U), F, k)$ (see $\S 2.3$ and Definition 2.20 for the definition of $\left.\langle-\mid-\rangle^{\mathrm{SS}}(\varphi(U), F, k)\right)$. The local index ind ${ }_{z}^{\mathrm{PH}} \sigma$ is well defined by [49, Lemma 26]. Then the Poincaré-Hopf Euler number is defined to be the sum of the local indices:

Definition 2.24. The Poincaré-Hopf Euler number $n^{\mathrm{PH}}(V, \sigma)$ of $V$ with respect to $\sigma$ is $n^{\mathrm{PH}}(V, \sigma)=\sum_{z: \sigma(z)=0} \operatorname{ind}_{z}^{\mathrm{PH}} \sigma$.

Proof of Theorem 1.1. By Proposition 2.3 we have $n^{\mathrm{GS}}(V)=n^{\mathrm{GS}}(V, \sigma)$, where the orientation has been suppressed from the notation but is indeed present. Using equation (3), it is thus enough to show that $\operatorname{ind}_{z}(\sigma)=\operatorname{ind}_{z}^{\mathrm{PH}}(\sigma)$. This follows from Theorem 2.18. One needs to be careful about the trivializations used in defining the various pushforward maps; this is ensured precisely by the condition that the tautological section is a square. The details of this argument are spelled out more carefully in the proof of Proposition 3.13.

One can extend the comparison of local degrees $\operatorname{ind}_{z}^{\mathrm{PH}} \sigma=\operatorname{ind}_{z} \sigma$ to work over a more general base scheme $S$. This was done for $S=\mathbb{A}_{k}^{1}$ with $k$ a field in [49, Lemma 33], but in more generality, it is useful to pick the local coordinates using knowledge of both $\sigma$ and $X$, as follows:

Definition 2.25. Let $X$ be a scheme, $V$ a vector bundle on $X$, and $\sigma$ a section of $V$.

(1) We call $\sigma$ nondegenerate if it locally corresponds to a regular sequence.

(2) Given another scheme $S$ and a morphism $\pi: X \rightarrow S$, we call $\sigma$ very nondegenerate (with respect to $\pi$ ) if it is nondegenerate and the zero locus $Z(\sigma)$ is finite and locally free over $S$.

Remark 2.26. Suppose that $X$ is smooth over $S$ and $r k(V)=\operatorname{dim} X / S$.

(1) If $S=\operatorname{Spec}(k)$ is the spectrum of a field, then $Z(\sigma) \rightarrow \operatorname{Spec}(k)$ is quasi-finite if and only if it is finite locally free, if and only if $\sigma$ is locally given by a regular sequence. 
In other words, $\sigma$ is nondegenerate if and only if it is very nondegenerate, if and only if $Z(\sigma) \rightarrow Z$ is quasi-finite.

(2) In general, $\sigma$ is nondegenerate as soon as $Z(\sigma) \rightarrow S$ is quasi-finite, and very nondegenerate if and only if $Z(\sigma) \rightarrow S$ is finite (see Lemma B.5).

Example 2.27. If $\sigma$ is a nondegenerate section, then precomposition with $\sigma$ induces an isomorphism $\operatorname{Hom}(V, \mathcal{O}) \simeq C_{Z / X}$. In particular, $N_{Z / X} \simeq V$ and $L_{Z / X} \simeq V^{*}[1]$.

Definition 2.28. Let $X$ be a smooth $S$-scheme and let $V \rightarrow X$ be a vector bundle, relatively oriented by $\rho$. Let $\sigma$ be a very nondegenerate section of $V$ and let $Z$ be a closed and open subscheme of the zero locus $Z(\sigma)$ of $\sigma$. By a system of coordinates for $(V, X, \sigma, \rho, Z)$ we mean an open neighborhood $U$ of $Z$ in $X$, an étale map $\varphi: U \rightarrow \mathbb{A}_{S}^{n}$, a trivialization $\psi:\left.V\right|_{U} \simeq \mathcal{O}_{U}^{n}$, and a section $\sigma^{\prime} \in \mathcal{O}_{\mathbb{A}_{S}^{n}}^{n}(\varphi(U))$, such that the following conditions hold:

(1) $Z=Z\left(\left.\sigma\right|_{U}\right) \cong Z\left(\sigma^{\prime}\right)$,

(2) $\operatorname{det}\left(\left.\sigma\right|_{Z}\right)=\operatorname{det}\left(\left.\varphi^{*} \sigma^{\prime}\right|_{Z}\right) \in \operatorname{det} N_{Z / X}$, and

(3) the canonical section of $\left.\left.\omega_{X / S} \otimes \operatorname{det} V\right|_{Z} \cong \mathcal{L}^{\otimes 2}\right|_{Z}$ determined by $\psi$ and $\varphi$ corresponds to the square of a section of $\left.\mathcal{L}\right|_{Z}$.

Here for conditions (2) and (3) we used Example 2.27.

Suppose that $X$ has dimension $n$ over $S$, so that the rank of $V$ is also $n$. Let $Z \subset Z(\sigma)$ be a clopen component and write $\varpi: Z \rightarrow S$ for the structure map. The local index generalizes straightforwardly from Definition 2.14:

Definition 2.29. We call

$$
\operatorname{ind}_{Z}(\sigma)=\operatorname{ind}_{Z}(V, \sigma, \rho)=\varpi_{*}\left(i^{*} \mathcal{L} \otimes i^{*} \mathcal{L} \rightarrow i^{*} \mathcal{L}^{\otimes 2}\right) \in \operatorname{BL}^{\text {naive }}(S)
$$

the local index at $Z$.

Remark 2.30. Since $\varpi$ is finite locally free, $\varpi_{*}$ preserves vector bundles. In particular, $\operatorname{ind}_{Z}(\sigma) \in \mathrm{BL}^{\text {naive }}(S)$ is a symmetric bilinear form on a vector bundle, as opposed to just on a complex up to homotopy. (See also Remark 2.10.)

A system of coordinates for $(V, X, \sigma, \rho, Z)$ determines a presentation $Z=Z\left(\left.\sigma\right|_{U}\right)=$ $Z\left(\sigma^{\prime}\right) \subset \mathbb{A}_{S}^{n}$, where $\sigma^{\prime}: \mathbb{A}_{S}^{n} \supset \phi(U) \rightarrow \mathbb{A}^{n}$. Hence Definition 2.20 supplies us with a symmetric bilinear form $\langle-\mid-\rangle^{\mathrm{SS}}(\varphi(U), \sigma, S) \in \mathrm{BL}^{\text {naive }}(S)$.

Proposition 2.31. The form $\langle-\mid-\rangle^{\mathrm{SS}}\left(\varphi(U), \sigma^{\prime}, S\right)$ coincides (up to isomorphism) with $\operatorname{ind}_{Z}(\sigma)$. In particular, its isomorphism class is independent of the choice of coordinates.

Proof. The argument is the same as in the proofs of Theorem 1.1 and Proposition 3.13.

In contrast, our proof that the Euler number (sum of indices) is independent of the choice of section (i.e., Proposition 2.3) does not generalize immediately; in fact, this will not hold in $\mathrm{BL}^{\text {naive }}(S)$ but rather in some quotient (like $\mathrm{GW}(k)$ in the case of fields). As 
indicated in Remark 2.7, one situation in which it is easy to see this independence is if the quotient group satisfies homotopy invariance. This suggests studying Euler numbers valued in more general homotopy-invariant cohomology theories for algebraic varieties, which is what the remainder of this work is concerned with.

\section{Cohomology theories for schemes}

\subsection{Introduction}

In order to generalize the results from the previous sections, we find it useful to introduce the concept of a cohomology theory twisted by K-theory. We do not seek here to axiomatize all the relevant data, just to introduce a common language for similar phenomena.

Definition 3.1. Let $S$ be a scheme and $\mathcal{C} \subset \operatorname{Sch}_{S}$ a category of schemes. Denote by $\mathcal{C}^{L}$ the category of pairs $(X, \xi)$, where $X \in \mathcal{C}$ and $\xi \in K(X)$ (i.e., a point in the $K$-theory space of $X$ ), and morphisms those maps of schemes compatible with the $K$-theory points. ${ }^{5}$ By a cohomology theory $E$ over $S$ (for schemes in $\mathcal{C}$ ) we mean a presheaf of sets on $\mathcal{C}^{L}$-that is, a functor

$$
E:\left(\mathcal{C}^{L}\right)^{\text {op }} \rightarrow \operatorname{Set},(X, \xi) \mapsto E^{\xi}(X) .
$$

To illustrate the flavor of cohomology theory we have in mind, we begin with two examples.

Example 3.2. We can set either of the following:

(1) $E^{\xi}(X)=\mathrm{CH}^{r k(\xi)}(X)$, the Chow group of algebraic cycles up to rational equivalence of the appropriate codimension, or

(2) $E^{\xi}(X)=\mathrm{GW}(X, \widetilde{\operatorname{det} \xi})$, the Grothendieck-Witt group of symmetric bilinear perfect complexes for the duality $\underline{\operatorname{Hom}}(-, \widetilde{\operatorname{det}} \xi)$ (see, e.g., [68]).

Warning 3.3. For cohomology theories with values in a 1-category (like sets), in this definition we can safely replace $K(X)$ by its truncation $K(X)_{\leq 1}$-that is, the ordinary 1-groupoid of virtual vector bundles. However, we can in general not replace it by just the set $K_{0}(X)$. In other words, if (say) $V$ is a vector bundle on $X$ and $\phi$ an automorphism of $V$, then there is an induced automorphism

$$
E(\phi): E^{V}(X) \rightarrow E^{V}(X)
$$

which may or may not be trivial. For example, in the case $E=\mathrm{GW}$ as before, if $V=\mathcal{O}$ is trivial and $\phi$ corresponds to $a \in \mathcal{O}^{\times}(X)$, then $E(\phi)$ is given by multiplication by $\langle a\rangle \in$ $\mathrm{GW}(X)$.

\footnotetext{
${ }^{5}$ Technically speaking, this means 'coherently compatible', so $\mathcal{C}^{L}$ is an $\infty$-category. However, we will only need its homotopy 1-category, so for us 'compatible' means 'together with a homotopy class of paths joining the two $K$-theory points'.
} 


\subsection{Features of cohomology theories}

Many cohomology theories that occur in practice satisfy additional properties beyond the basic ones of our definition, and many come with more data. We list here some of those relevant to this paper.

Morphisms of theories: Cohomology theories form a category in an evident way, with morphisms given by natural transformations.

Trivial bundles: We usually abbreviate $E^{\mathcal{O}^{n}}(X)$ to $E^{n}(X)$.

Additive and multiplicative structure: Often, $E$ takes values in abelian groups. Moreover, often $E^{0}(X)$ is a ring and $E^{\xi}(X)$ is a module over $E^{0}(X)$. Typically all of this structure is preserved by the pullback maps.

Disjoint unions: Usually $E$ converts finite disjoint unions into products-that is, $E(\emptyset)=*$ and $E(X \coprod Y)=E(X) \times E(Y)$. If $E$ takes values in abelian groups, this is usually written as $E(X \coprod Y)=E(X) \oplus E(Y)$.

Orientations: In many cases, the cohomology theory $E$ factors through a quotient of the category $\mathcal{C}^{L}$, built using a quotient $q: K(X) \rightarrow K^{\prime}(X)$ of the $K$-theory groupoid. In other words, one has canonical isomorphisms $E^{\xi}(X) \simeq E^{\xi^{\prime}}(X)$ for certain $K$ theory points $\xi, \xi^{\prime}$. More specifically:

GL-orientations: If $K^{\prime}(X)=\mathbb{Z}$ and $q$ is the rank map, then we speak of a GL-orientation. In other words, in this situation we canonically have $E^{\xi}(X) \simeq$ $E^{r k(\xi)}(X)$. In particular, Warning 3.3 does not apply: all automorphisms of vector bundles act trivially on $E$. This happens, for example, if $E=\mathrm{CH}$ (see Example $3.2(1))$.

SL-orientations: If instead $K^{\prime}(X)=\operatorname{Pic}(D(X))$ via the determinant, then we speak of an SL-orientation. In other words, in this situation $E^{\xi}(X)$ depends only on the rank and (ungraded) determinant of $\xi$. We write $E^{r k(\xi)}(X, \operatorname{det}(\xi))$ for this common group. This happens, for example, if $E=\mathrm{GW}$ (see Example 3.2(1)).

$\mathrm{SL}^{c}$-orientations: This is a further strengthening of the concept of an SLorientation, where in $K^{\prime}(X)=\operatorname{Pic}(D(X))$ we mod out (in the sense of groupoids) by the squares of line bundles. In other words, if $\mathcal{L}_{1}, \mathcal{L}_{2}, \mathcal{L}_{3}$ are line bundles on $X$, then any isomorphism $\mathcal{L}_{1} \simeq \mathcal{L}_{2} \otimes \mathcal{L}_{3}^{\otimes 2}$ induces

$$
E^{n}\left(X, \mathcal{L}_{1}\right) \simeq E^{n}\left(X, \mathcal{L}_{2}\right)
$$

Note that then, in particular, $E^{n}(X, \mathcal{L}) \simeq E^{n}\left(X, \mathcal{L}^{*}\right)$. This also happens for $E=$ GW, essentially by construction.

Supports: Often, for $Z \subset X$ closed there is a cohomology with support, denoted $E_{Z}^{\xi}(X)$. It enjoys further functorialities which we do not list in detail here.

Transfers: In many theories, for appropriate morphisms $p: X \rightarrow Y$ and $\xi \in K(Y)$, there exists $t w(p, \xi) \in K(X)$ and a transfer map

$$
p_{*}: E^{t w(p, \xi)}(X) \rightarrow E^{\xi}(Y)
$$


compatible with composition. Typically $p$ is required to be lci, and

$$
t w(p, \xi)=p^{*} \xi+L_{p}
$$

where $L_{p}$ is the cotangent complex [46]. Furthermore, typically $p$ is required to be proper, or else we need to fix $Z \subset X$ closed and proper over $Y$ and obtain $p_{*}$ : $E_{Z}^{t w(p, \xi)}(X) \rightarrow E^{\xi}(Y)$. Finally, usually $E$ takes values in abelian groups and satisfies the disjoint union property, and transfer from a disjoint union is just the sum of the transfers.

\section{Remark 3.4.}

(1) We have defined a morphism of cohomology theories as a natural transformation of functors valued in sets. Whether or not such a transformation respects additional structure (abelian group structures, orientations, transfers, etc.) must be investigated in each case.

(2) In many cases (in particular in the presence of homotopy invariance), SL-oriented theories are also canonically $\mathrm{SL}^{c}$-oriented (see Proposition 4.19).

\subsection{Some cohomology theories}

We now introduce a number of cohomology theories that can be used in this context.

Hermitian $\boldsymbol{K}$-theory GW: This is the theory from Example 3.2(2). It is $\mathrm{SL}^{c}-$ oriented. We believe that it has transfers for (at least) smooth, proper morphisms and regular immersions, but we are not aware of a reference for this in adequate generality. If $X$ is regular and $1 / 2 \in X$, one can use the comparison with KO-theory (see later).

Naive derived bilinear forms $\mathrm{BL}_{\text {naive: }}$ See $\S 2.2$.

Cohomology theories represented by motivic spectra: Let $\mathcal{S H}(S)$ denote the motivic stable $\infty$-category. Then any $E \in \mathcal{S H}(S)$ defines a cohomology theory on $\mathrm{Sch}_{S}$, automatically satisfying many good properties; for example, they always have transfers along smooth and proper morphisms, as well as regular immersions. For a lucid introduction, see [31]. We recall some of the main points in $\S 4$.

Orthogonal $\boldsymbol{K}$-theory spectrum KO: This spectrum is defined and stable under arbitrary base change if $1 / 2 \in S[71,65]$. Over regular bases, it represents Hermitian $K$-theory $\mathrm{GW}$; in general it represents a homotopy-invariant version.

Generalized motivic cohomology $H \tilde{\mathbb{Z}}$ : This can be defined as $\pi_{0}^{\text {eff }}(\mathbb{1})$ (see, e.g., [5]). Over fields (of characteristic not 2) it represents generalized motivic cohomology in the sense of Calmès and Fasel [20, 9, 20]; it is unclear whether this theory is useful in this form over more general bases.

\subsection{The yoga of Euler numbers}

Let $E$ be a cohomology theory. 
Definition 3.5. We will say that $E$ has Euler classes if, for each scheme $X$ over $S$ and each vector bundle $V$ on $X$, we are supplied with a class

$$
e(V, E) \in E^{V^{*}}(X) \text {. }
$$

Remark 3.6. The twist by $V^{*}$ (instead of $V$ ) in this definition may seem peculiar. It ultimately comes from our choice of covariant (instead of contravariant) equivalence between locally free sheaves and vector bundles, whereas a contravariant equivalence is used in the motivic Thom spectrum functor and hence in the definition of twists.

Typically, the Euler classes will satisfy further properties, such as stability under base change; we do not formalize this here.

Now suppose that $\pi: X \rightarrow S$ is smooth and proper, $V$ is relatively oriented, and $E$ has transfers for smooth proper maps and is $\mathrm{SL}^{c}$-oriented. In this case we have a transfer map

$$
E^{V^{*}}(X) \simeq E^{n}(X, \operatorname{det} V) \stackrel{\rho}{\simeq} E^{n}\left(X, \omega_{X / S}\right) \simeq E^{L_{\pi}}(X) \stackrel{\pi_{*}}{\longrightarrow} E^{0}(S)
$$

Definition 3.7. In the foregoing situation, we call

$$
n(V, \rho, E)=\pi_{*} e(V, E)
$$

the Euler number of $V$ in $E$ with respect to the relative orientation $\rho$.

Example 3.8. Let $E=\mathrm{GW}$. We can define a family of Euler classes by

$$
e(V)=[K(V, 0)] \in \mathrm{GW}(X, \widetilde{\operatorname{det} V}) \simeq \mathrm{GW}^{V^{*}}(X) ;
$$

here we use the Koszul complex from $§ 2.1$. This depends initially on a choice of section, but we shall show that the Grothendieck-Witt class often does not. In any case, here we chose the zero section for definiteness. Assuming that GW has transfers (of the expected form) in this context, we find that

$$
n(V, \rho, \mathrm{GW})=n^{\mathrm{GS}}(V, 0, \rho) .
$$

Now let $\sigma$ be a nondegenerate section of $V$ (in the sense of Definition 2.25) and write $i: Z=Z(\sigma) \hookrightarrow X$ for the inclusion of the zero scheme. Thus $i$ is a regular immersion. In this case one has (see Example 2.27)

$$
\left[L_{i}\right] \simeq-\left[N_{Z / X}^{*}\right] \simeq-\left[\left.V^{*}\right|_{Z}\right]
$$

and consequently, if $E$ has push-forwards along regular immersions, there is a transfer map

$$
i_{*}: E^{0}(Z) \simeq E^{\left.\left[V^{*}\right]\right|_{z}-\left[V^{*} \mid z\right]}(Z) \simeq E^{i^{*} V^{*}+L_{i}}(Z) \rightarrow E^{V^{*}}(X) .
$$

The following result is true in all cases that we know of; but of course it cannot be proved from the weak axioms that we have listed:

Meta-Theorem 3.9. Let $\sigma$ be a nondegenerate section of a vector bundle $V$ over a scheme $X$. Let $E$ be a cohomology theory with Euler classes and push-forwards along 
regular immersions, such that $E^{0}(S)$ has a distinguished element 1 (e.g., is a ring). Then

$$
e(V, E)=i_{*}(1)
$$

where we use the identification from before for the push-forward.

Going back to the situation where $X$ is smooth and proper over $S, V$ is relatively oriented, and $E$ is $\mathrm{SL}^{c}$-oriented and has transfers along proper lci morphisms, we also have the push-forward

$$
\varpi_{*}: E^{0}(Z) \simeq E^{0}\left(Z,\left.\mathcal{L}^{\otimes 2}\right|_{Z}\right) \stackrel{\rho}{\simeq} E^{0}\left(Z,\left.\operatorname{det} V^{*} \otimes \omega_{X / S}\right|_{Z}\right) \simeq E^{L_{\varpi}}(Z) \rightarrow E^{0}(S) .
$$

More generally, if $Z^{\prime} \subset Z$ is a clopen component, then we have a similar transfer originating from $E^{0}\left(Z^{\prime}\right)$.

Definition 3.10. For $V, \sigma, X, E$ as before, for any clopen component $Z^{\prime} \subset Z$ we denote by

$$
\operatorname{ind}_{Z^{\prime}}(\sigma, \rho, E)=\varpi_{*}^{\prime}(1) \in E^{0}(S)
$$

the local index of $\sigma$ around $Z^{\prime}$ in $E$. Here $\varpi^{\prime}: Z^{\prime} \rightarrow S$ is the restriction of $\varpi$ to $Z^{\prime}$.

Meta-Corollary 3.11. Let $\sigma$ be a nondegenerate section of a relatively oriented vector bundle $V$ over $\pi: X \rightarrow S$. Let $E$ be an $\mathrm{SL}^{c}$-oriented cohomology theory with Euler classes and push-forwards along proper lci morphisms, such that Meta-Theorem 3.9 applies. Then

$$
n(V, \rho, E)=\sum_{Z^{\prime}} \operatorname{ind}_{Z^{\prime}}(\sigma, \rho, E) .
$$

Proof. By assumption, transfers are compatible with composition and additive along disjoint unions. The result follows.

Example 3.12. If $S=\operatorname{Spec}(k)$ is the spectrum of a field, then $Z$ is 0 -dimensional and hence decomposes into a finite disjoint union of 'fat points'. In particular, the Euler number is expressed as a sum of local indices, in bijection with the zeros of our nondegenerate section.

Recall the notion of coordinates from Definition 2.28. The following result states that indices may be computed in local coordinates:

Proposition 3.13. Let $E$ be an $\mathrm{SL}^{c}$-oriented cohomology theory with Euler classes and push-forwards along proper lci morphisms. Let $\left(\psi, \varphi, \sigma_{2}\right)$ be a system of coordinates for $\left(V, X, \sigma_{1}, \rho_{1}, Z\right)$. Then

$$
\operatorname{ind}_{Z}\left(\sigma_{1}, \rho_{1}, E\right)=\operatorname{ind}_{Z}\left(\sigma_{2}, \rho_{2}, E\right),
$$

where $\rho_{2}$ is the canonical relative orientation of $\mathcal{O}_{\mathbb{A}^{n}}^{n} / \mathbb{A}^{n}$.

Proof. Let $\varpi: Z \rightarrow S$ denote the canonical map. Then both sides are obtained as $\varpi_{*}(1)$, but conceivably the orientations used to define the transfer could be different; we shall show that they are not. In other words, we are given two isomorphisms

$$
\widetilde{\operatorname{det}} L_{\varpi} \stackrel{\alpha_{i}}{\simeq} \mathcal{L}_{i}^{\otimes 2}
$$


and we need to exhibit $\mathcal{L}_{1} \stackrel{\beta}{\simeq} \mathcal{L}_{2}$ such that $\alpha_{1}^{-1} \alpha_{2}=\beta^{\otimes 2}$. The isomorphisms $\alpha_{i}$ arise as

$$
\left.\left.\left.\operatorname{det} L_{\varpi} \simeq \operatorname{det} N_{Z / X} \otimes \omega_{X / S}\right|_{Z} \stackrel{\sigma_{i}}{\simeq} \operatorname{det} V \otimes \omega_{X / S}\right|_{Z} \stackrel{\rho_{i}}{\simeq} \mathcal{L}_{i}^{\otimes 2}\right|_{Z}
$$

where $\mathcal{L}_{2}=\mathcal{O}$ and for $i=2$ we implicitly use $\varphi$ and $\psi$ as well. We first check that the two isomorphisms $\left.\operatorname{det} N_{Z / X} \simeq \operatorname{det} V\right|_{Z}$ are the same. Indeed, $\left.V\right|_{U} \simeq \mathcal{O}^{n}$ via $\psi$, and up to this trivialization the isomorphism is given by the trivialization of $C_{Z / X}$ by $\sigma_{i}$; these are the same by Definition 2.28. Now we deal with the second half of the isomorphism. By

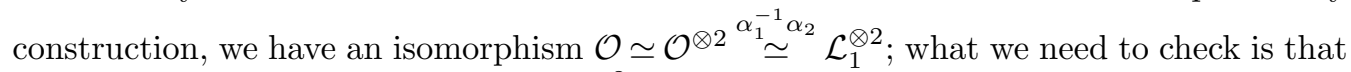
the corresponding global section of $\mathcal{L}_{1}^{\otimes 2}$ is a tensor square. Unwinding the definitions, this follows from Definition 2.28.

\section{Cohomology theories represented by motivic spectra}

We recall some background material about motivic extraordinary cohomology theoriesthat is, theories represented by motivic spectra. We make essentially no claim to originality.

\subsection{Aspects of the six-functors formalism}

We recall some aspects of the six-functors formalism for the motivic stable categories $\mathcal{S H}(-)$, following the exposition in [31].

4.1.1. Adjunctions. For every scheme $X$, we have a symmetric monoidal, stable category $\mathcal{S H}(X)$. For every morphism $f: X \rightarrow Y$ of schemes we have an adjunction

$$
f^{*}: \mathcal{S H}(Y) \leftrightarrows \mathcal{S H}(X): f_{*}
$$

If no confusion can arise, we sometimes write $E_{Y}:=f^{*} E$. If $f$ is smooth, there is a further adjunction

$$
f_{\#}: \mathcal{S H}(X) \leftrightarrows \mathcal{S H}(Y): f^{*}
$$

If $f$ is locally of finite type, then there is the exceptional adjunction

$$
f_{!}: \mathcal{S H}(X) \leftrightarrows \mathcal{S H}(Y): f^{!}
$$

There is a natural transformation $\alpha: f_{!} \rightarrow f_{*}$. If $f$ is proper, then $\alpha$ is an equivalence.

The assignments $f \mapsto f^{*}, f_{*}, f^{!}, f_{!}, f_{\#}$ are functorial. In particular, given composable morphisms $f, g$ of the appropriate type, we have equivalences $(f g)_{*} \simeq f_{*} g_{*}$, and so on. 
4.1.2. Exchange transformations. Suppose a commutative square of categories

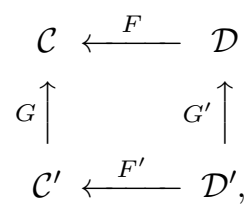

which is a natural isomorphism $\gamma: F G^{\prime} \simeq G F^{\prime}$. If the functors $G^{\prime}, G$ have right adjoints $H^{\prime}, H$, then we have the natural transformation

$$
F^{\prime} H^{\prime} \stackrel{\text { unit }}{\longrightarrow} H G F^{\prime} H^{\prime} \stackrel{\gamma}{\simeq} H F G^{\prime} H^{\prime} \stackrel{\text { counit }}{\longrightarrow} H F,
$$

called the associated exchange transformation. Similarly, if $G^{\prime}, G$ have left adjoints $K^{\prime}, K$, then we have the exchange transformation

$$
K F \stackrel{\text { unit }}{\longrightarrow} K F G^{\prime} K^{\prime} \stackrel{\gamma}{\simeq} K G F K^{\prime} \stackrel{\text { counit }}{\longrightarrow} F K^{\prime}
$$

Suppose we have a commutative square of schemes

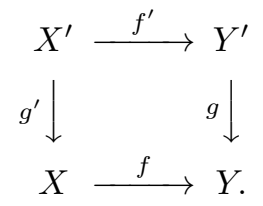

Then we have an induced commutative square of categories

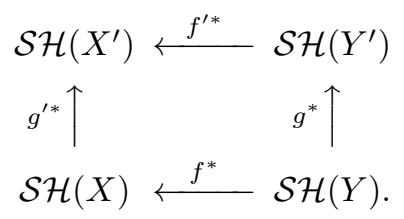

Passing to the right adjoints, we obtain the exchange transformation

$$
\mathrm{Ex}_{*}^{*}: f^{*} g_{*} \rightarrow g_{*}^{\prime} f^{\prime *} \text {. }
$$

Similarly, there is $\operatorname{Ex}_{\#}^{*}: g_{\#}^{\prime} f^{\prime *} \rightarrow f^{*} g_{\#}$ (for $g$ smooth; this is in fact an equivalence if diagram (4) is cartesian), and so on.

4.1.3. Exceptional exchange transformation. Given a cartesian square of schemes as in diagram (4), with $g$ (and hence $g^{\prime}$ ) locally of finite type, there is a canonical equivalence

$$
\operatorname{Ex}_{!}^{*}: f^{*} g_{!} \simeq g_{!}^{\prime} f^{\prime *}
$$

Passing to right adjoints, we obtain

$$
\mathrm{Ex}^{* !}: f^{\prime *} g^{!} \rightarrow g^{\prime !} f^{*}
$$

4.1.4. Thom transformation. Given a perfect complex $\mathcal{E}$ of vector bundles on $X$, the motivic J-homomorphism $K(X) \rightarrow \operatorname{Pic}(\mathcal{S H}(X))[10, \S 16.2]$ provides us with an invertible $\operatorname{spectrum} \Sigma^{\mathcal{E}} \mathbb{1} \in \mathcal{S H}(X)$. We denote by $\Sigma^{\mathcal{E}}: \mathcal{S H}(X) \rightarrow \mathcal{S H}(X), E \mapsto E \wedge \Sigma^{\mathcal{E}} \mathbb{1}$ the associated 
invertible endofunctor. If $\mathcal{E}$ is a vector bundle (concentrated in degree 0 ), then $\Sigma^{\mathcal{E}} \mathbb{1}$ is the suspension spectrum on the Thom space $\mathcal{E}^{*} / \mathcal{E}^{*} \backslash 0{ }^{6}$

Lemma 4.1. The functor $f^{!}$commutes with Thom transforms.

Proof. This follows from the projection formula [23, A.5.1(6)] and the invertibility of $\Sigma^{(-)} \mathbb{1}$ :

$$
f^{!}\left(\Sigma^{V} X\right) \simeq f^{!} \underline{\operatorname{Hom}}\left(\Sigma^{-V} \mathbb{1}, X\right) \simeq \underline{\operatorname{Hom}}\left(f^{*} \Sigma^{-V} \mathbb{1}, f^{!} X\right) \simeq \Sigma^{f^{*} V} f^{!} X
$$

4.1.5. Purity transformation. Let $f: X \rightarrow Y$ be a smoothable [31, §2.1.21] lci morphism. Then the cotangent complex $\mathrm{L}_{f}$ is perfect, and there exists a canonical purity transformation

$$
\mathfrak{p}_{f}: \Sigma^{\mathrm{L}_{f}} f^{*} \rightarrow f^{!}
$$

\subsection{Cohomology groups and Gysin maps}

4.2.1. Let $S$ be a scheme and set $E \in \mathcal{S H}(S)$. Given $(\pi: X \rightarrow S) \in \operatorname{Sch}_{S}, i: Z \hookrightarrow$ $X$ closed, and $\xi \in K(Z)$, we define the $\xi$-twisted E-cohomology of $X$ with support in $Z$ as

$$
E_{Z}^{\xi}(X)=\left[\mathbb{1}, \pi_{*} i_{!} \Sigma^{\xi} i^{!} \pi^{*} E\right]_{\mathcal{S H}(S)}
$$

This assignment forms a cohomology theory in the sense of $§ 3.1$. It takes values in abelian groups, has supports, and satisfies the disjoint union property. We shall see that it has transfers for proper lci maps. It need not be orientable in general.

If $Z=X$, we may omit it from the notation and just write $E^{\xi}(X)$. As before, if $\xi$ is a trivial virtual vector bundle of rank $n \in \mathbb{Z}$, then we also write $E_{Z}^{n}(X)$ instead of $E_{Z}^{\xi}(X)$.

Example 4.2. Suppose that $\xi=i^{*} V$, where $V$ is a vector bundle on $X$. We have

$$
E_{Z}^{\xi}(X)=\left[\mathbb{1}, \pi_{*} i_{!} \Sigma^{\xi} i^{!} \pi^{*} E\right]_{\mathcal{S H}(S)} \simeq\left[\mathbb{1}, i^{!} \Sigma^{\xi} \pi^{*} E\right]_{\mathcal{S H}(Z)} \simeq\left[i_{*} \mathbb{1}, \Sigma^{\xi} \pi^{*} E\right]_{\mathcal{S H}(X)},
$$

where we have used the fact that $i_{*} \simeq i_{\text {! }}$ and Lemma 4 .1. Using the localization sequence $j_{\#} j^{*} \rightarrow \mathrm{id} \rightarrow i_{*} i^{*}[44$, Theorem $6.18(4)]$ to identify $i_{*} \mathbb{1} \simeq X / X \backslash Z$, we find that

$$
E_{Z}^{\xi}(X) \simeq\left[X /(X \backslash Z), \Sigma^{\xi} \pi^{*} E\right]_{\mathcal{S H}(X)} .
$$

Remark 4.3. The final expression in Example 4.2 depends only on $Z \subset X$ as a subset, not a subscheme. This also follows directly from the definition, since $\mathcal{S H}(Z) \simeq \mathcal{S H}\left(Z_{\text {red }}\right)$; this is another consequence of localization.

\footnotetext{
${ }^{6}$ Indeed, in [31, §2.1.2], the transformation $\Sigma^{\mathcal{E}}$ is built out of $\operatorname{Spec}(\operatorname{Sym}(\mathcal{E}))$-which is the vector bundle corresponding to $\mathcal{E}^{*}$ in our convention.
} 
4.2.2. Functoriality in $\boldsymbol{E}$. If $\alpha: E \rightarrow F \in \mathcal{S H}(S)$ is any morphism, then there is an induced morphism $\alpha_{*}: E_{Z}^{\xi}(X) \rightarrow F_{Z}^{\xi}(X)$. This just follows from the fact that $\pi^{*}$ and so on are functors.

4.2.3. Contravariant functoriality in $\boldsymbol{X}$. Define $f: X^{\prime} \rightarrow X \in \operatorname{Sch}_{S}$. Then there is a pullback map

$$
f^{*}: E_{Z}^{\xi}(X) \rightarrow E_{f^{-1}(Z)}^{f^{*} \xi}\left(X^{\prime}\right)
$$

coming from the morphism

$$
\begin{gathered}
\pi_{*} i_{!} \Sigma^{\xi} i^{!} \pi^{*} E \stackrel{\text { unit }}{\longrightarrow} \pi_{*} f_{*} f^{*} i_{!} \Sigma^{\xi} i^{!} \pi^{*} E \simeq \pi_{*}^{\prime} f^{*} i_{!} \Sigma^{\xi} i^{!} \pi^{*} E \stackrel{\mathrm{Ex}_{!}^{*}}{\simeq} \pi_{*}^{\prime} i_{!}^{\prime} f^{\prime *} \Sigma^{\xi} i^{!} \pi^{*} E \\
\simeq \pi_{*}^{\prime} i_{!}^{\prime} \Sigma^{f^{*} \xi} f^{\prime *} i^{!} \pi^{*} E \stackrel{\mathrm{Ex}}{\longrightarrow} \pi_{*}^{\prime} i_{!}^{\prime} \Sigma^{f^{*} \xi} i^{\prime *} f^{*} \pi^{*} E \simeq \pi_{*}^{\prime} i_{!}^{\prime} \Sigma^{f^{*} \xi} i^{\prime *} \pi^{\prime *} E .
\end{gathered}
$$

Here the $\mathrm{Ex}_{\text {! }}^{*}$ and $\mathrm{Ex}^{*}$ ! come from the cartesian square

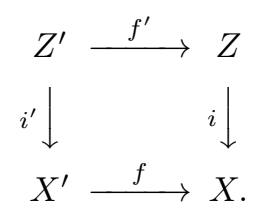

Lemma 4.4. Set $f: X^{\prime} \rightarrow X \in \operatorname{Sch}_{X}, Z \subset X, \xi \in K(Z)$, and $\alpha: E \rightarrow F \in \mathcal{S H}(S)$. The following square commutes:

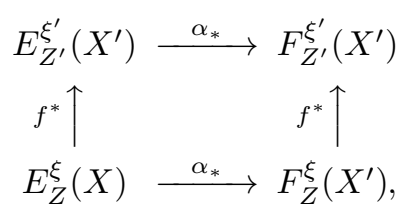

where $\xi^{\prime}=f^{*}(\xi)$ and $Z^{\prime}=f^{-1}(Z)$.

Proof. This is just an expression of the fact that the exchange transformations used to build $f^{*}$ are indeed natural transformations.

4.2.4. Covariant functoriality in $\boldsymbol{X}$. Suppose a commutative square in $\operatorname{Sch}_{S}$,

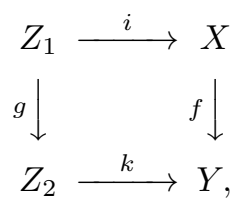

where $f$ is smoothable lci, $i, k$ are closed immersions, and $g$ is proper. For every $\xi \in K\left(Z_{2}\right)$, there is a Gysin map

$$
f_{*}: E_{Z_{1}}^{g^{*} \xi+i^{*} L_{f}}(X) \rightarrow E_{Z_{2}}^{\xi}(Y)
$$


coming from the morphism

$$
\begin{aligned}
f_{*} i_{!} \Sigma^{g^{*} \xi+i^{*} L_{f}} i^{!} f^{*} E_{Y} & \stackrel{\mathfrak{p}_{f}}{\longrightarrow} f_{*} i_{!} \Sigma^{g^{*} \xi} i^{!} f^{!} E_{Y} \simeq k_{!} g_{!} \Sigma^{g^{*} \xi} g^{!} k^{!} E_{Y} \simeq k_{!} g_{!} g^{!} \Sigma^{\xi} k^{!} E_{Y} \\
& \stackrel{\text { counit }}{\longrightarrow} k_{!} \Sigma^{\xi} k^{!} E_{Y}
\end{aligned}
$$

where we used Lemma 4.1 to move $\Sigma^{L_{f}}$ through $i^{!}$and $\Sigma^{\xi}$ through $g^{!}$, and also used $i_{*} \simeq i_{!}, k_{*} \simeq k_{!}, g_{*} \simeq g_{!}$.

Remark 4.5. In [31], the Gysin map is denoted by $f_{!}$, to emphasize that it involves the purity transform. We find the notation $f_{*}$ more convenient.

Lemma 4.6. The following hold:

(1) Suppose a square as in diagram (5) and $\alpha: E \rightarrow F \in \mathcal{S H}(S)$. Then the following square commutes:

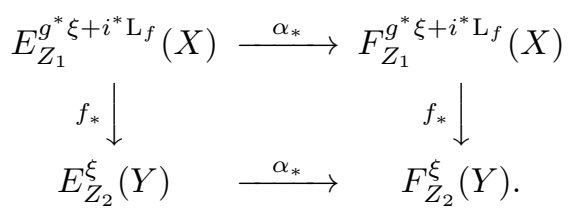

(2) Suppose a square as in diagram (5) and $s: Y^{\prime} \rightarrow Y$ such that $s, f$ are tor-independent. Set $X^{\prime}=X \times_{Y} Y^{\prime}, Z_{i}^{\prime}=Z_{i} \times_{Y} Y^{\prime}, i^{\prime}, g^{\prime}, k^{\prime}, f^{\prime}$ the induced maps, and so on. Then the following square commutes:

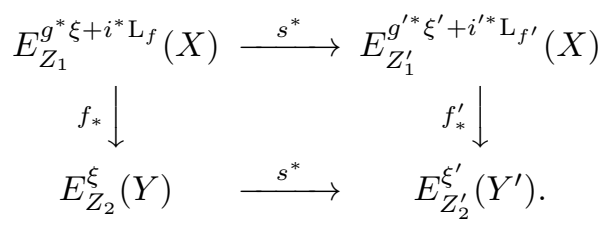

(3) Suppose a commutative diagram in $\mathrm{Sch}_{S}$ as follows

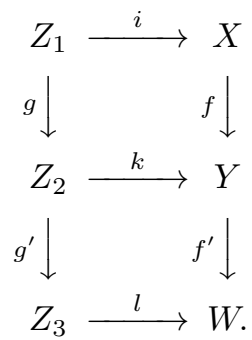


Then given $\xi \in K\left(Z_{3}\right)$, we have

$$
f_{*}^{\prime} f_{*}=\left(f^{\prime} f\right)_{*}: E_{Z_{1}}^{\left(g^{\prime} g\right)^{*} \xi+i^{*} L_{f^{\prime} f}}(X) \rightarrow E_{Z_{3}}^{\xi}(W)
$$

Here we use the equivalence $L_{f^{\prime} f} \simeq f^{\prime *} L_{f}+L_{f^{\prime}} \in K(X)$, coming from the cofiber sequence $f^{\prime *} L_{f} \rightarrow L_{f^{\prime} f} \rightarrow L_{f^{\prime}}$.

Proof. (1) Since $f_{*}: E_{Z_{1}}^{g^{*} \xi+i^{*} L_{f}}(X) \rightarrow E_{Z_{2}}^{\xi}(Y)$ is obtained as [1, $\left.\zeta_{E}\right]$, where $\zeta$ is a natural transformation of endofunctors of $\mathcal{S H}(S)$, this is clear.

(2) Consider the following diagram:

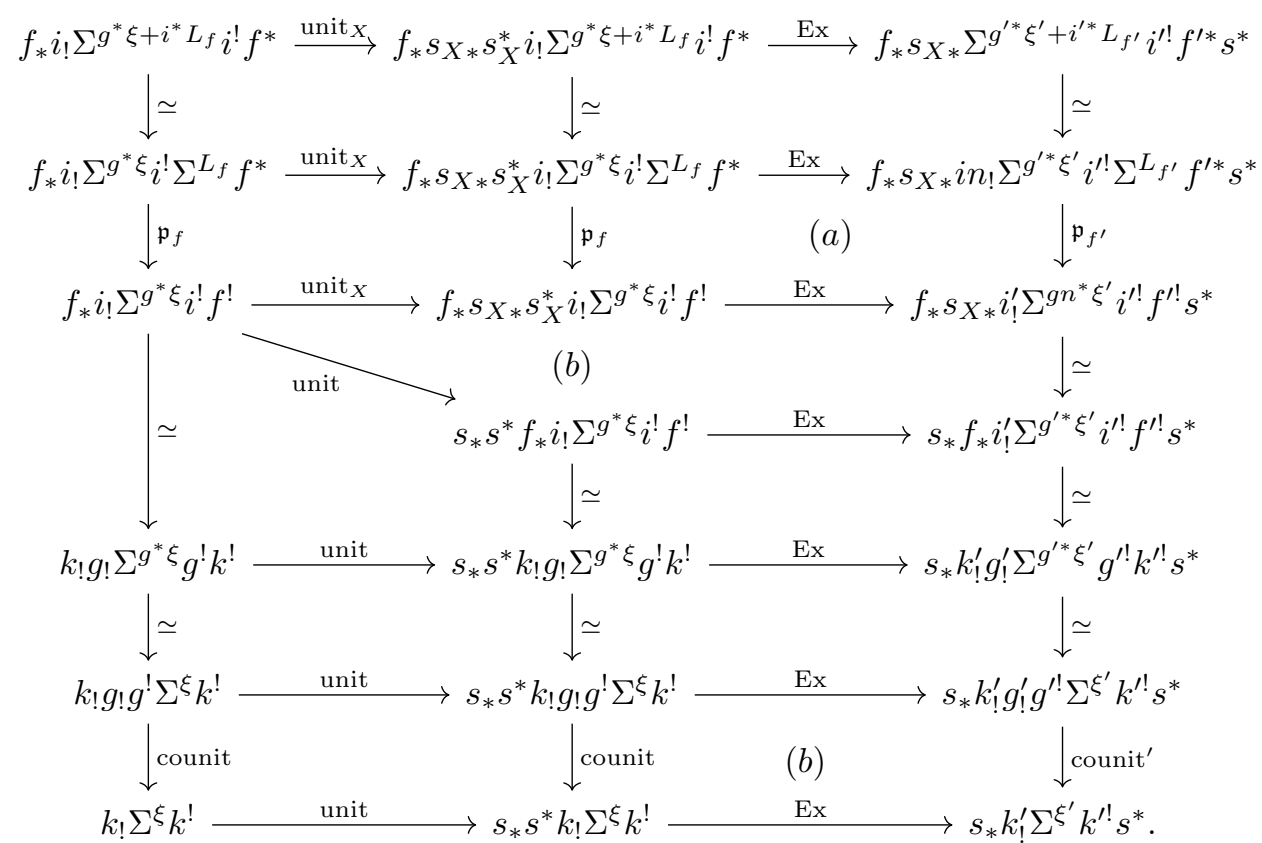

Here $s_{X}: X^{\prime} \rightarrow X$ is the canonical map and $\xi^{\prime}=\left(Z_{2}^{\prime} \rightarrow Z_{2}\right)^{*} \xi$. All the unlabeled equivalences arise from moving Thom transforms through (various) pullbacks, the compatibility of pullbacks and push-forwards with composition, and equivalences of the form $p_{*} \simeq p_{\text {! for }} p$ proper. All the maps labeled 'Ex' are exchange transformations expressing the compatibility of $p_{*}, p_{!}, p^{!}$with base change. Denote the diagram by $\mathcal{D}$. Then $\left[\mathbb{1}, \mathcal{D} E_{Y}\right]$ yields a diagram of abelian groups. The outer square of that diagram identifies with the square which we are trying to show commutes. It thus suffices to show that $\mathcal{D}$ commutes. All cells commute for trivial reasons, except for (a), which commutes by [31, Proposition 2.2.2(ii)] and (b), which commute by the stability of the counit transformations under base change. 
(3) Consider the following diagram:

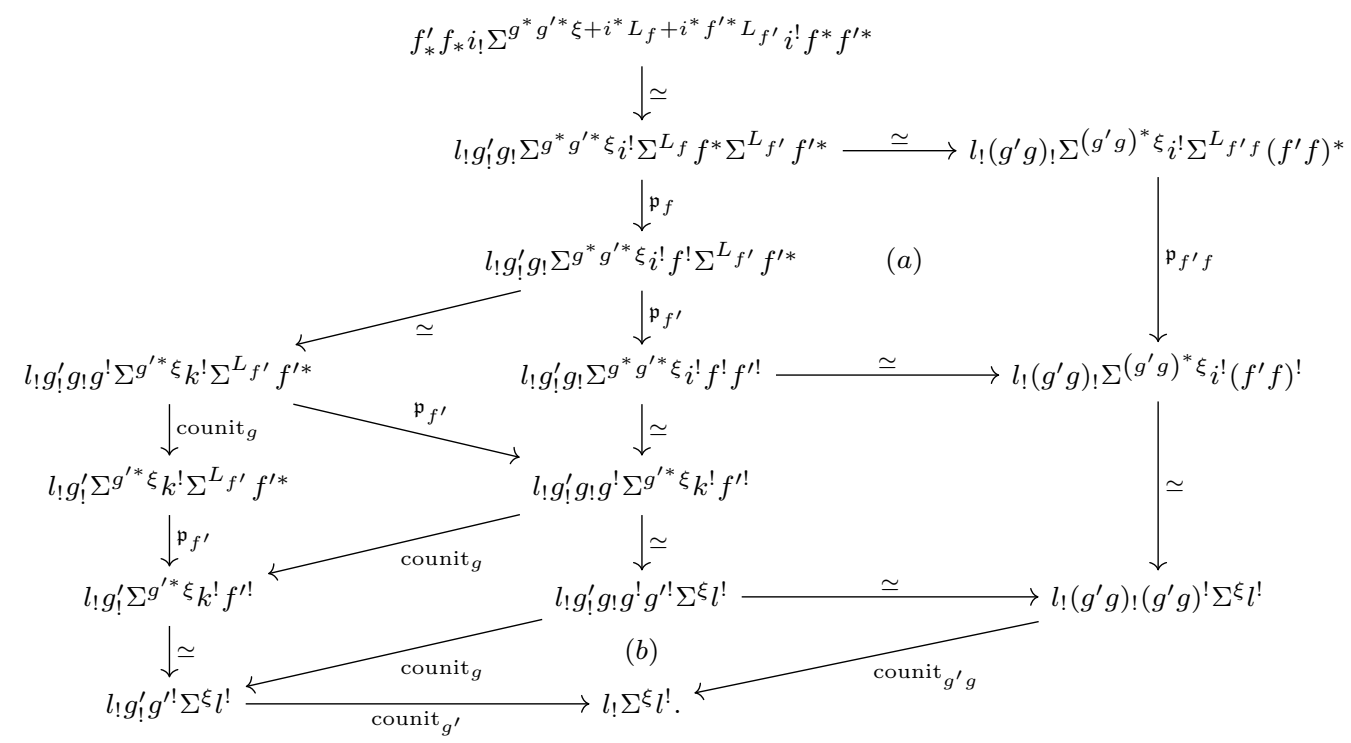

All the unlabeled equivalences arise from moving Thom transforms through (various) pullbacks, the compatibility of pullbacks and push-forwards with composition, and equivalences of the form $p_{*} \simeq p_{\text {! for }} p$ proper. Denote the diagram by $\mathcal{D}$. Then $\left.\mathbb{1}, \mathcal{D} E_{W}\right]$ yields a diagram of abelian groups. Going from the top to the bottom middle via the leftmost path, we obtain $f_{*}^{\prime} f_{*}$; going instead via the rightmost path we obtain $\left(f^{\prime} f\right)_{*}$. It hence suffices to show that $\mathcal{D}$ commutes. All cells commute for trivial reasons, except for (a), which commutes by [31, Proposition 2.2.2(i)], and (b), which commutes by the compatibility of the counit transformations with composition.

Example 4.7. Consider a commutative square as in diagram (5), with $X=Y$ and $f=\mathrm{id}$, so that $g: Z_{1} \hookrightarrow Z_{2}$ is a closed immersion. Then $f_{*}: E_{Z_{1}}^{g^{*} \xi}(X) \rightarrow E_{Z_{2}}^{\xi}(X)$ is the 'extension of support' map. In particular taking $Z_{2}=X$ as well, we obtain the map $E_{Z_{1}}^{i^{*} \xi}(X) \rightarrow E^{\xi}(X)$ 'forgetting the support'. Lemma 4.6(3) now in particular tells us that given a proper map $f: X \rightarrow Y$, a closed immersion $i: Z \hookrightarrow X$, and $\xi \in K(Y)$, the following diagram commutes:

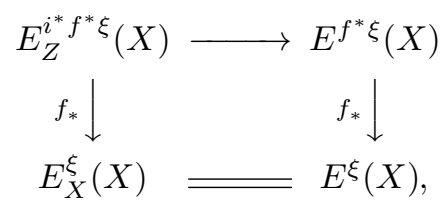

where the upper horizontal map forgets the support.

\subsection{Orientations}

4.3.1. Product structures. By a ring spectrum (over $S$ ) we an object $E \in \mathcal{S H}(S)$ together with homotopy classes of mapsu $: \mathbb{1} \rightarrow E$ and $m: E \wedge E \rightarrow E$ satisfying the 
evident identities. If $E$ is a ring spectrum, then there are multiplication maps

$$
E_{Z_{1}}^{\xi}(X) \times E_{Z_{2}}^{\xi^{\prime}}(X) \rightarrow E_{Z_{1} \cap Z_{2}}^{\xi+\xi^{\prime}}(X)
$$

induced by

$$
\left(\Sigma^{\xi} E\right) \wedge\left(\Sigma^{\xi^{\prime}} E\right) \simeq \Sigma^{\xi+\xi^{\prime}} E \wedge E \stackrel{m}{\longrightarrow} \Sigma^{\xi+\xi^{\prime}} E
$$

and the diagonal ${ }^{7}$

$$
X / X \backslash\left(Z_{1} \cap Z_{2}\right) \rightarrow X / X \backslash Z_{1} \wedge X / X \backslash Z_{2} .
$$

Lemma 4.8. The multiplicative structure on E-cohomology is compatible with pullback: given $Z_{1}, Z_{2} \subset X, \xi, \xi^{\prime} \in K(X)$, and $f: X^{\prime} \rightarrow X$, the following diagram commutes:

$$
\begin{array}{rlc}
E_{Z_{1}}^{\xi}(X) \times E_{Z_{2}}^{\xi^{\prime}}(X) & \longrightarrow E_{Z_{1} \cap Z_{2}}^{\xi+\xi^{\prime}}(X) \\
f^{*} \times f^{*} \downarrow & f^{*} \downarrow \\
E_{f^{-1}\left(Z_{1}\right)}^{f^{*} \xi}\left(X^{\prime}\right) \times E_{f^{-1}\left(Z_{2}\right)}^{f^{*} \xi^{\prime}}\left(X^{\prime}\right) & \longrightarrow E_{f^{-1}\left(Z_{1}\right) \cap f^{-1}\left(Z_{2}\right)}^{f^{*} \xi+f^{*} \xi^{\prime}}\left(X^{\prime}\right) .
\end{array}
$$

Proof. This is immediate from the definitions.

4.3.2. Thom spectra. Let $G=\left(G_{n}\right)_{n}$ be a family of finitely presented $S$-group schemes, equipped with a morphism of associative algebras $G \rightarrow\left(\mathrm{GL}_{n k, S}\right)_{n}$ (for the Day convolution symmetric monoidal structure on $\left.\operatorname{Fun}\left(\mathbb{N}, \operatorname{Grp}\left(\operatorname{Sch}_{S}\right)\right)\right)$. Then there is a notion of a (stable) vector bundle with structure group $G$, the associated $K$-theory space $K^{G}(X)$, and the associated Thom spectrum $\mathrm{MG}$, which is a ring spectrum [10, Example 16.22].

Example 4.9. If $G_{n}=\mathrm{GL}_{n}$, then $K^{G}(X)=K(X)$ and MGL is the algebraic cobordism spectrum [10, Theorem 16.13]. If $G_{n}=\mathrm{SL}_{n}$ (resp., $\mathrm{Sp}_{n}$ ), then $K^{G}(X)$ is the $K$-theory of oriented (resp., symplectic) vector bundles in the usual sense, and MSL (resp., MSp) is the Thom spectrum as defined in [63].

In order to work effectively with $\mathrm{M} G$, one needs to know that it is stable under base change. This is easily seen to be true for MGL, MSL, and MSp [10, Example 16.23]. We record the following more general result for future reference:

Proposition 4.10. The Thom spectrum MG is stable under base change, provided that each $G_{n}$ is flat and quasi-affine.

Proof. We have a presheaf $K^{G} \in \mathcal{P}\left(\operatorname{Sch}_{S}\right)$ and a map $K^{G} \rightarrow K$. For $f: X \rightarrow S \in \operatorname{Sch}_{S}$, denote by $K_{X}^{G} \in \mathcal{P}\left(\operatorname{Sm}_{X}\right)$ and $j_{X}:\left.K_{X}^{G} \rightarrow K\right|_{\operatorname{Sm}_{X}}$ the restrictions. Then by definition, $\mathrm{M} G_{X}=M_{X}\left(j_{X}\right)$, where $M_{X}: \mathcal{P}\left(\operatorname{Sm}_{X}\right) / K \rightarrow \mathcal{S H}(X)$ is the motivic Thom-spectrum functor $[10, \S 16.1]$. Let $L K_{S}^{G} \in \mathcal{P}\left(\mathrm{Sch}_{S}\right)$ denote the left Kan extension of $K_{S}^{G}$. We claim that $L K_{S}^{G} \rightarrow K^{G}$ is a Nisnevich equivalence. Assuming this, we deduce that $\left.f^{*} K_{S}^{G} \simeq\left(L K_{S}^{G}\right)\right|_{\operatorname{Sm}_{X}} \rightarrow K_{X}^{G}$ is a Nisnevich equivalence. Since $M_{X}$ inverts Nisnevich

\footnotetext{
${ }^{7}$ One easily checks that the diagonal $X \rightarrow X \times X$ induces a map as indicated.
} 
equivalences [10, Proposition 16.9], this implies that $f^{*} \mathrm{M} G_{S} \simeq \mathrm{M} G_{X}$, which is the desired result.

To prove the claim, we first note that by [32, Lemma 3.3.9], we may assume $S$ is affine, and it suffices to prove that the restriction of $K^{G}$ to $\mathrm{Aff}_{S}$ is left Kan extended from smooth affine $S$-schemes. By definition, $K^{G}=\left(\text { Vect }^{G}\right)^{g p}$, where $\operatorname{Vect}^{G}=\coprod_{n \geq 0} B G_{n}$ (here the coproduct is as stacks i.e., fppf sheaves). The desired result now follows from [32, Proposition A.0.4 and Lemma A.0.5] (noting that the coproduct of stacks is the same as the coproduct of $\Sigma$-presheaves, and Kan extension preserves $\Sigma$-presheaves).

Now set $\xi \in K^{G}(X)$. Then there is a canonical equivalence [10, Example 16.29]

$$
\Sigma^{\xi} \mathrm{M} G_{X} \simeq \Sigma^{|\xi|} \mathrm{M} G_{X} .
$$

We denote by $t_{\xi} \in \mathrm{M} G^{\xi-|\xi|}(X)$ the class of the map

$$
\mathbb{1} \stackrel{u}{\rightarrow} \mathrm{M} G_{X} \simeq \Sigma^{\xi-|\xi|} \mathrm{M} G_{X} .
$$

\subsubsection{Oriented ring spectra.}

Definition 4.11. Let $E \in \mathcal{S H}(S)$ be a ring spectrum and $G=\left(G_{n}\right)_{n}$ a family of group schemes as in $\S 4.3 .2$. By a strong $G$-orientation of $E$ we mean a ring map $\mathrm{M} G \rightarrow E$.

Example 4.12. The spectrum KO is strongly SL-oriented (see Corollary A.3).

Note that if $E \in \mathcal{S H}(S)$ is strongly $G$-oriented, then there is no reason a priori why $E_{X}$ should be strongly $G_{X}$-oriented. This is true if $\mathrm{M} G$ is stable under base change, so for most reasonable $G$ by Proposition 4.10. We will not talk about strong $G$-orientations unless $\mathrm{M} G$ is stable under base change, so assume this throughout.

Given $\xi \in K^{G}(X)$, the map $\mathrm{M} G \rightarrow E$ provides us with $t_{\xi}=t_{\xi}(E) \in E^{\xi-|\xi|}(X)$.

Proposition 4.13. Let $E$ be strongly $G$-oriented and set $\xi \in K^{G}(X)$.

(1) The classes $t_{\xi}(E)$ are stable under base change: for $f: X^{\prime} \rightarrow X$ we have $t_{f^{*} \xi}(E)=$ $f^{*} t_{\xi}(E)$.

(2) Multiplication by $t_{\xi}(E)$ induces an equivalence $\Sigma^{|\xi|} E \simeq \Sigma^{\xi} E$ and an isomorphism $t: E_{Z}^{|\xi|}(X) \simeq E_{Z}^{\xi}(X)$, called the Thom isomorphism.

(3) The Thom isomorphism is compatible with base change: $f^{*}(t(x))=t\left(f^{*}(x)\right)$.

In particular, E is G-oriented.

Proof. (1) follows from the same statement for $M G$, where it holds by construction. For the first half of $(2)$, it suffices to show that $t_{\xi}(E)$ is a unit in the Picard-graded homotopy ring of $E$. This follows from the same statement for $M G$. The second half of (2) follows. (3) immediately follows from (1).

Example 4.14. Let $E$ be strongly GL-oriented. Then for any $\xi \in K(X)$ we obtain $E^{\xi}(X) \simeq E^{r k(\xi)}(X)$, so $E$ is oriented in the sense of $\S 3.2$. 
Definition 4.15. For $X \in \operatorname{Sch}_{S}$ and $\mathcal{L}$ a line bundle on $X$, put

$$
E_{Z}^{n}(X, \mathcal{L})=E_{Z}^{n-1+\mathcal{L}}(X)
$$

Example 4.16. Let $E$ be strongly SL-oriented and set $\xi \in K(X)$. Then $\xi^{\prime}:=\xi-(|\xi|-1+$ $\operatorname{det} \xi) \in K(X)$ lifts canonically to $K^{\mathrm{SL}}(X)$, whence by Proposition 4.13 we get a canonical (Thom) isomorphism $E^{\xi}(X) \simeq E^{|\xi|}(X, \operatorname{det} \xi)$. In particular, $E$ is SL-oriented in the sense of $\S 3.2$.

Remark 4.17. If $E$ is strongly SL-oriented, then $\operatorname{since} \operatorname{det}\left(\mathcal{L}_{1} \oplus \mathcal{L}_{2}\right) \simeq \mathcal{L}_{1} \otimes \mathcal{L}_{2}$, by Example 4.16 the product structure on $E$-cohomology twisted by line bundles takes the form $E^{n}\left(X, \mathcal{L}_{1}\right) \times E^{m}\left(X, \mathcal{L}_{2}\right) \rightarrow E^{n+m}\left(X, \mathcal{L}_{1} \otimes \mathcal{L}_{2}\right)$.

Remark 4.18. Strong $G$-orientations have better permanence properties than ordinary ones (provided that $\mathrm{M} G$ is stable under base change): they are stable under base change and taking (very) effective covers, for example.

\section{4. $S L^{c}$-orientations}

A. Ananyevskiy has done important work on SL and $\mathrm{SL}^{c}$ orientations. We shall make use of the following result (see, e.g., [1, Theorem 1.1]):

Proposition 4.19 (Ananyevskiy). Let $E \in \mathcal{S H}(S)$ be SL-oriented and $\mathcal{L}_{1}, \mathcal{L}_{2}, \mathcal{L}_{3}$ be line bundles on $X$. Suppose an isomorphism $\mathcal{L}_{1} \simeq \mathcal{L}_{2} \otimes \mathcal{L}_{3}^{\otimes 2}$.

(1) There is a canonical equivalence $\Sigma^{\mathcal{L}_{1}} E \simeq \Sigma^{\mathcal{L}_{2}} E$, compatible with base change.

(2) There is a canonical isomorphism $E_{Z}^{n}\left(X, \mathcal{L}_{1}\right) \simeq E_{Z}^{n}\left(X, \mathcal{L}_{2}\right)$, compatible with base change.

In particular, the cohomology theory represented by $E$ is $\mathrm{SL}^{c}$-oriented.

Proof. Note that (2) follows from (1). Let $\mathcal{L}=\mathcal{L}_{3}$. It suffices to exhibit a canonical equivalence $\Sigma^{\mathcal{L}^{\otimes 2}} E \simeq \Sigma^{\mathcal{O}} E$. We have canonical equivalences

$$
\Sigma^{\mathcal{L}+\mathcal{L}} E \simeq \Sigma^{\mathcal{O}+\mathcal{L}^{\otimes 2}} E, \quad \Sigma^{\mathcal{L}+\mathcal{L}^{*}} E \simeq \Sigma^{\mathcal{O}^{2}} E, \quad \Sigma^{\mathcal{L}+\mathcal{L}} \simeq \Sigma^{\mathcal{L}+\mathcal{L}^{*}}
$$

by [1, Corollary 3.9, Lemma 4.1]. Consequently, $\Sigma^{\mathcal{O}+\mathcal{L}^{\otimes 2}} E \simeq \Sigma^{\mathcal{O}^{2}} E$, whence the claim.

\section{Euler classes for representable theories}

\subsection{Tautological Euler class}

Let $E \in \mathcal{S H}(S)$ be a ring spectrum, set $X \in \operatorname{Sch}_{S}$, and let $V$ be a vector bundle on $X$.

Definition 5.1. We denote by $e(V)=e(V, E) \in E^{V^{*}}(X)$ the tautological Euler class of $V$, defined as the composite

$$
\mathbb{1}_{X} \simeq \Sigma_{+}^{\infty} V \rightarrow \Sigma^{\infty} V /\left.(V \backslash 0) \simeq \Sigma^{V^{*}} \mathbb{1} \stackrel{u}{\rightarrow} \Sigma^{V^{*}} E\right|_{X} \in \mathcal{S H}(X) .
$$

Lemma 5.2. Define $f: X^{\prime} \rightarrow X \in \operatorname{Sch}_{S}$. Then

$$
f^{*} e(V, E)=e\left(f^{*} V, E\right) \in E^{f^{*} V}\left(X^{\prime}\right) .
$$


Proof. This is immediate.

If $E$ is strongly SL-oriented in the sense of $\S 4.3 .3$, and hence $\mathrm{SL}^{c}$-oriented in the sense of $\S 3.2$, then for any relatively oriented vector bundle $V$ over a smooth and proper scheme $X / S$ we obtain an Euler number $n(V, \rho, E) \in E^{0}(S)$ (see $\S 3.4$ ).

\subsection{Integrally defined Euler numbers}

Corollary 5.3 (Euler numbers are stable under base change). Let $E$ be a strongly SLoriented cohomology theory and let $V$ be vector bundle $V$ over a smooth and proper scheme $X / S$, relatively oriented by $\rho$. Let $f: S^{\prime} \rightarrow S$ be a morphism of schemes. Then

$$
f^{*} n(V, \rho, E)=n\left(f^{*} V, f^{*} o, f^{*} E\right) \in E^{0}\left(S^{\prime}\right) .
$$

Proof. This holds because all our constructions are stable under base change-see in particular Lemma 4.6(2) (for the compatibility of Gysin maps with pullback, which applies since $X \rightarrow S$ is smooth), Propositions 4.19 and 4.13(3) (ensuring that the identification $E^{V^{*}}(X) \simeq E^{L_{\pi}}(X)$ is compatible with base change), and Lemma 5.2 (for the compatibility of Euler classes with base change).

Proposition 5.4. Let $d$ be even or $d=1, X / \mathbb{Z}[1 / d]$ smooth and proper, and $V / X$ a relatively oriented vector bundle. Then for any field $k$ with $2 d \in k^{\times}$, we have

$$
n\left(V_{k}, \rho, \mathrm{H} \tilde{\mathbb{Z}}\right) \in \mathbb{Z}[\langle-1\rangle,\langle 2\rangle, \ldots,\langle d\rangle] \subset \mathrm{GW}(k) .
$$

In fact, there is a formula

$$
n\left(V_{k}, \rho, \mathrm{H} \tilde{\mathbb{Z}}\right)=\sum_{a \in \mathbb{Z}[1 / d !] \times} n_{a}\langle a\rangle,
$$

which holds over any such field, with the coefficients $n_{a} \in \mathbb{Z}$ independent of $k$ (and zero for all but finitely many a).

Remark 5.5. If $d=1$, Proposition 5.4 relies on the novel results about Hermitian $K$ theory of the integers from [18]. In the following proof, this is manifested in the dependence of $[9$, Lemma $3.38(2)]$ on these results. We will later use Proposition 5.4 for the $d=1$ case of Theorem 5.11, whence this result is also using [18] in an essential way. For $d \geq 2$, the proof is independent of [18].

Note that here the assumption that the rank of $V$ equals the dimension of $X$ is included in the hypothesis that $V / X$ is a relatively oriented vector bundle (see Definition 1.5).

Proof. Recall the very effective cover functor $\tilde{f}_{0}$ and the truncation in the effective homotopy $t$-structure $\pi_{0}^{\text {eff }}$, for example from $[5, \S \S 3,4]$. We have a diagram of spectra

$$
\mathrm{KO}_{k} \leftarrow \tilde{f}_{0} \mathrm{KO}_{k} \rightarrow \pi_{0}^{\mathrm{eff}} \mathrm{KO}_{k} \leftarrow \pi_{0}^{\mathrm{eff}} \mathrm{MSL}_{k} \simeq H \tilde{\mathbb{Z}}
$$

see [10, Example 16.34] for the last equivalence. The functors $\tilde{f}_{0}$ and $\pi_{0}^{\text {eff }}$ are lax monoidal in an appropriate sense, so this is a diagram of ring spectra. Moreover, all of the ring spectra are strongly SL-oriented (via the ring map $\mathrm{MSL}_{k} \rightarrow \pi_{0}^{\text {eff }} \mathrm{MSL}_{k}$; see also Remark 
4.18). Finally, all the maps induce isomorphisms on $[\mathbb{1},-]$, essentially by construction. It follows that $n\left(V_{k}, \rho, \mathrm{KO}\right)=n\left(V_{k}, \rho, H \tilde{\mathbb{Z}}\right) \in \mathrm{GW}(k)$. We may thus just as well prove the result for $n\left(V_{k}, \rho, \mathrm{KO}\right)$ instead.

If $d$ is even, then we have $\mathrm{KO} \in \mathcal{S H}(\mathbb{Z}[1 / d])$, and by Corollary 5.3 we see that $n\left(V_{k}, \rho, \mathrm{KO}\right) \in i m(\mathrm{GW}(\mathbb{Z}[1 / d]) \rightarrow \mathrm{GW}(k))$. The result thus follows from Lemma 5.6. If $d=1$, we use the $\mathrm{SL}$-oriented ring spectrum $\mathrm{KO}^{\prime} \in \mathcal{S H}(\mathbb{Z})$ from $[9, \S 3.8 .3]$. We find that $n\left(V_{k}, \rho, \mathrm{KO}\right)$ is the image of $n\left(V, \rho, \mathrm{KO}^{\prime}\right) \in \mathrm{KO}^{\prime}(\mathbb{Z})$. This latter group is isomorphic to $\mathrm{GW}(\mathbb{Z})$ by $[9$, Lemma $3.38(2)]$, whence the result.

Lemma 5.6. Let $d$ be even or $d=1$. As a ring, $\mathrm{GW}(\mathbb{Z}[1 / d])$ is generated by $\langle-1\rangle$ and $\langle p\rangle$, for the primes $p \mid d$.

Proof. Let $A=\mathbb{Z}[1 / d]$ and $U=\operatorname{Spec} A$. If $d=1$, this is well known [59, Theorem II.4.3]. Hence from now on, $1 / 2 \in A$. Using Lemma B.2, it suffices to prove the analogous statement for $W(A)$. Let $I \subset W$ be the fundamental ideal and denote by $I^{*}$ its powers; we view these as presheaves on $U$. Consider the commutative graded ring $k_{*}=H_{\mathrm{Nis}}^{*}\left(U, I^{*} / I^{*+1}\right)$. Of course $k_{0}=\mathbb{Z} / 2$. It follows from [11, Corollary 4.9], [7, Theorem 2.1, Lemma 2.7], and [71, Theorem 3.9] that $k_{1} \simeq \mathcal{O}^{\times}(U) / 2$ and $k_{n} \simeq \mathbb{Z} / 2\left\{(-1)^{n}\right\}$ for $n \geq 2$. We have the classes $\langle a\rangle-1 \in I(U)$ (for $a \in \mathcal{O}^{\times}(U)$ ) showing that $H_{\mathrm{Nis}}^{0}\left(U, I^{n}\right) \rightarrow k_{n}$ is surjective. The short exact sequences

$$
0 \rightarrow H_{\mathrm{Nis}}^{0}\left(U, I^{n+1}\right) \rightarrow H_{\mathrm{Nis}}^{0}\left(U, I^{n}\right) \rightarrow k_{n}
$$

thus show that $H_{\mathrm{Nis}}^{0}(U, W)$ is generated by the $\langle a\rangle$ together with $H_{\mathrm{Nis}}^{0}\left(U, I^{n}\right)$, for any $n$. For $n$ sufficiently large, $H_{\text {Nis }}^{0}\left(U, I^{n}\right) \simeq 2^{n} H_{\text {ret }}^{0}(U, \mathbb{Z}) \simeq 2^{n} \mathbb{Z}[7$, Proposition 2.3] is generated by $(\langle-1\rangle-1)^{n}$; thus $H_{\mathrm{Nis}}^{0}(U, W)$ is generated by the $\langle a\rangle$. It remains to observe that $H_{\text {Nis }}^{0}(U, W)=W(U)$. This follows from the descent spectral sequence for computing $W(U)=[U, \mathrm{KW}]$, using the fact that the motivic spectrum $\mathrm{KW}$ has $\underline{\pi}_{*} \mathrm{KW}=a_{\mathrm{Nis}} W$ for $* \equiv 0(\bmod 4)$ and $\underline{\pi}_{*} \mathrm{KW}=0$.

Notation 5.7. Set $A \hookrightarrow \mathbb{R}$ and let $V$ be a relatively oriented, rank $n$ vector bundle on a smooth, proper $n$-dimensional scheme $X$ over $A$. We have $\mathrm{GW}(\mathbb{R}) \simeq \mathbb{Z} \oplus \mathbb{Z}\langle-1\rangle$. There are thus unique integers $n_{\mathbb{R}}, n_{\mathbb{C}} \in \mathbb{Z}$ such that

$$
n\left(V_{\mathbb{R}}, \rho, H \tilde{\mathbb{Z}}\right)=\frac{n_{\mathbb{C}}+n_{\mathbb{R}}}{2}+\frac{n_{\mathbb{C}}-n_{\mathbb{R}}}{2}\langle-1\rangle
$$

The integers $n_{\mathbb{R}}$ and $n_{\mathbb{C}}$ are the Euler numbers of the corresponding real and complex topological vector bundles, respectively, at least when $X$ is projective, justifying the notation. To show this, consider the cycle class map $\mathrm{CH}^{*}(X) \rightarrow H^{*}(X(\mathbb{C}), \mathbb{Z})$ from the Chow ring of a smooth $\mathbb{C}$-scheme $X$ to the singular cohomology of the complex manifold $X(\mathbb{C})[37$, Chapter 19]. Furthermore, there are real cycle class maps from oriented Chow rings of $\mathbb{R}$-smooth schemes $X$ to the singular cohomology of the real manifold $X(\mathbb{R})$, discussed in [43] (as well as more refined real cycle class maps defined in [13]): for a smooth $\mathbb{R}$-scheme $X$ and a line bundle $\mathcal{L} \rightarrow X$, consider the real cycle class map

$$
\widetilde{\mathrm{CH}}^{*}(X, \mathcal{L}) \cong H^{n}\left(X, \mathrm{~K}_{n}^{\mathrm{MW}}\left(\omega_{X / k}\right)\right) \cong H \tilde{\mathbb{Z}}^{L_{\pi}}(X) \rightarrow H^{*}(X(\mathbb{C}), \mathbb{Z}(\mathcal{L}))
$$


from the oriented Chow groups twisted by $\mathcal{L}$ to the singular homology of the associated local system on $X(\mathbb{R})$. We use results on the real cycle class map due to Hornbostel, Wendt, Xie, and Zibrowius [43], including compatibility with push-forwards. Since they only had need of this compatibility in the case of the push-forward by a closed immersion, we first extend it slightly:

Lemma 5.8. Let $\pi: X \rightarrow$ Spec $k$ be the structure map of a smooth, projective scheme $X$ of dimension $n$ over the real numbers $\mathbb{R}$. Then the following square commutes:

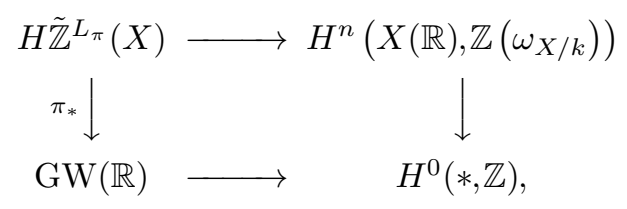

where the vertical maps are the canonical push-forwards and the horizontal maps are the real cycle class maps.

Proof. We have $\pi$ the composition of a closed immersion $i: X \hookrightarrow \mathbb{P}_{k}^{n}$ and the structure map $p: \mathbb{P}_{k}^{n} \rightarrow$ Spec $k$. The algebraic push-forward $\pi_{*}$ is the composition $p_{*} \circ i_{*}$, and the analogous statement holds for the topological push-forward by classical algebraic topology. By [43, Theorem 4.7], the push-forward $i_{*}$ commutes with real realization. We may thus reduce to the case where $X=\mathbb{P}_{k}^{n}$ is a projective $n$-space over a field $k$. In this case, $\pi_{*}$ is an isomorphism by [33, Prop 6.3, Theorem 11.7]. Let $s: \operatorname{Spec} K \rightarrow \mathbb{P}_{k}^{n}$ be the closed immersion given by the origin. Since $\pi s=1$, and the real realization maps commute with the algebraic and topological push-forwards of $s$ by [43, Theorem 4.7], the real realization maps also commute with the algebraic and topological pushforwards of $\pi$.

Proposition 5.9. Set $A \hookrightarrow \mathbb{R}$ and let $V$ be a relatively oriented, rank $n$ vector bundle on a smooth projective $n$-dimensional scheme $X$ over $A$. Then $n_{\mathbb{R}}$ and $n_{\mathbb{C}}$ are the Euler numbers of the corresponding real and complex topological vector bundles, respectively-that is, $n_{\mathbb{R}}$ is the topological Euler number of the relatively oriented topological $\mathbb{R}^{n}$-bundle $V(\mathbb{R})$ associated to the real points of $V$ on the real $n$-manifold $X(\mathbb{R})$, and $n_{\mathbb{C}}$ is the analogous topological Euler number on $V(\mathbb{C}) \rightarrow X(\mathbb{C})$.

Proof. By [43, Proposition 6.1], the $\mathbb{A}^{1}$-Euler class $e(V, H \tilde{\mathbb{Z}})$ of $V_{\mathbb{R}}$ in the oriented Chow group $H \tilde{\mathbb{Z}}^{V^{*}}\left(X_{\mathbb{R}}\right)$ maps to the topological Euler class of $V(\mathbb{R})$ under the real cycle class map. By Lemma 5.8, it follows that the image of the Euler number $n(V, H \tilde{\mathbb{Z}})$ under the real cycle class map is the topological Euler number of $V(\mathbb{R})$. Under the canonical isomorphism $H^{0}(*, \mathbb{Z}) \cong \mathbb{Z}$, the real cycle class map $\mathrm{GW}(\mathbb{R}) \rightarrow H^{0}(*, \mathbb{Z}) \cong \mathbb{Z}$ is the map taking a bilinear form over $\mathbb{R}$ to its signature. It follows that $n_{\mathbb{R}}$ is the topological Euler number of $V(\mathbb{R})$.

Let $\gamma: \widetilde{\mathrm{CH}^{*}}\left(X, \operatorname{det} V^{*}\right) \cong H \tilde{\mathbb{Z}}^{*}\left(X_{\mathbb{R}}\right) \rightarrow \mathrm{CH}^{*}(X) \rightarrow \mathrm{CH}^{*}\left(X_{\mathbb{C}}\right) \rightarrow H^{*}(X(\mathbb{C}), \mathbb{Z})$ denote the composition of the canonical map to Chow followed by the (usual) cycle class map, and 
we similarly have

$$
\gamma: \mathrm{GW}(\mathbb{R}) \cong \widetilde{\mathrm{CH}}^{0}(\operatorname{Spec} \mathbb{R}) \rightarrow H^{0}(\operatorname{Spec} \mathbb{R}(\mathbb{C}), \mathbb{Z}) \cong H^{0}(*, \mathbb{Z}) \cong \mathbb{Z},
$$

which sends the class of a bilinear form in $G W(\mathbb{R})$ to its rank. The cycle class map is compatible with Chern classes ([37, Proposition 19.1.2]), whence the image of $e(V, H \tilde{\mathbb{Z}})$ under $\gamma$ is the topological Euler class of $V(\mathbb{C})$. The cycle class map commutes with the relevant push-forwards and pullbacks [37, Corollary 19.2, Example 19.2.1], so we have that $\gamma(n(V, H \tilde{\mathbb{Z}}))=n_{\mathbb{C}}$ is the topological Euler number of $V(\mathbb{C})$.

Remark 5.10. If proper push-forwards in algebra and topology commute with real realization, as predicted by [43, 4.5 Remark], then Proposition 5.9 holds more generally for smooth, proper schemes. It seems that proving this would take us too far afield, however. Alternatively, if $V \otimes \mathbb{R}$ admits a nondegenerate section, then the results of $\S 8$ (showing that the Euler number can be computed in terms of Scheja-Storch forms) imply that Proposition 5.9 holds for smooth, proper schemes, arguing as in [72, Lemma 5].

Theorem 5.11. Suppose $X$ is smooth and proper over $\mathbb{Z}[1 / 2]$. Let $V$ be a relatively oriented vector bundle on $X$ and let $V_{k}$ denote the base change of $V$ to $k$ for any field $k$. Then one of the following is true:

$$
n^{\mathrm{GS}}\left(V_{k}, \rho\right)=\frac{n_{\mathbb{C}}+n_{\mathbb{R}}}{2}+\frac{n_{\mathbb{C}}-n_{\mathbb{R}}}{2}\langle-1\rangle
$$

or

$$
n^{\mathrm{GS}}\left(V_{k}, \rho\right)=\frac{n_{\mathbb{C}}+n_{\mathbb{R}}}{2}+\frac{n_{\mathbb{C}}-n_{\mathbb{R}}}{2}\langle-1\rangle+\langle 2\rangle-1,
$$

where the same formula holds for all fields $k$ of characteristic $\neq 2$.

If instead $X$ is smooth and proper over $\mathbb{Z}$, then equation (6) holds for any field $k$ (including fields $k$ of characteristic 2 ).

Recall that for the last claim regarding $X$ smooth and proper over $\mathbb{Z}$, we rely on [18] (see Remark 5.5).

Proof. First assume that $\operatorname{char}(k) \neq 2$. By Corollary 1.4 , we have $n^{\mathrm{GS}}\left(V_{k}, \rho\right)=$ $n\left(V_{k}, \rho, H \tilde{\mathbb{Z}}\right)$. If the base is $\mathbb{Z}$, then we learn from Proposition 5.4 that there exist $a, b \in \mathbb{Z}$ (independent of $k$ !) such that

$$
n\left(V_{k}, \rho, \mathrm{H} \tilde{\mathbb{Z}}\right)=a+b\langle-1\rangle .
$$

In $\mathrm{GW}(\mathbb{Z}[1 / 2]) \hookrightarrow \mathrm{GW}(\mathbb{Q})$ we have the relations

$$
\langle-2\rangle=1+\langle-1\rangle-\langle 2\rangle \quad \text { and } \quad 2\langle 2\rangle=2
$$

(the former because $\langle a\rangle+\langle-a\rangle=\langle 1\rangle+\langle-1\rangle$ for any $a$, and see, e.g., [6, Lemma 42] for the latter). Hence if the base is $\mathbb{Z}[1 / 2]$, there exist $a, b \in \mathbb{Z}, c \in\{0,1\}$ (independent of the choice of field $k !$ ) such that

$$
n\left(V_{k}, \rho, \mathrm{H} \tilde{\mathbb{Z}}\right)=a+b\langle-1\rangle+c\langle 2\rangle .
$$


If the base is $\mathbb{Z}$, let us put $c=0$. By construction we have

$$
n_{\mathbb{R}}=\operatorname{sign} n\left(V_{\mathbb{R}}, \rho, \mathrm{H} \tilde{\mathbb{Z}}\right)=(a+c)-b
$$

and

$$
n_{\mathbb{C}}=\operatorname{rank} n\left(V_{\mathbb{C}}, \rho, \mathrm{H} \tilde{\mathbb{Z}}\right)=(a+c)+b,
$$

which determines $a+c$ and $b$, so that there remain only at most two possible values for $n\left(V_{k}, \rho, \mathrm{H} \tilde{\mathbb{Z}}\right)$.

Now suppose that $\operatorname{char}(k)=2$ (so that in particular the base is $\mathbb{Z}$ ). Since $\langle 1\rangle=\langle-1\rangle$ over fields of characteristic 2 , we need to show that $n^{\mathrm{GS}}\left(V_{k}, \rho\right)=n_{\mathbb{C}}$. We may as well assume that $k=\mathbb{F}_{2}$. The rank induces an isomorphism $\mathrm{GW}\left(\mathbb{F}_{2}\right) \cong \mathbb{Z}$ (see, e.g., Corollary B.4). Considering the canonical maps

$$
\mathrm{GW}\left(\mathbb{F}_{2}\right) \rightarrow \mathrm{KGL}^{0}\left(\mathbb{F}_{2}\right) \leftarrow \mathrm{KGL}^{0}(\mathbb{Z}) \rightarrow \mathrm{KGL}^{0}(\mathbb{Z}[1 / 2]) \leftarrow \mathrm{GW}(\mathbb{Z}[1 / 2]),
$$

in which all but the right-most one are isomorphisms, we get the string of equalities

$$
n\left(V_{\mathbb{F}_{2}}\right)=n\left(V_{\mathbb{F}_{2}}, \mathrm{KGL}\right)=n\left(V_{\mathbb{Z}}, \mathrm{KGL}\right)=n\left(V_{\mathbb{Z}[1 / 2]}, \mathrm{KGL}\right)=r k\left(n\left(V_{\mathbb{Z}[1 / 2]}, \mathrm{KO}\right)\right) .
$$

The result follows.

Remark 5.12. The difference of the two values given in Theorem 5.11 is $\langle 2\rangle-1$, so the two possibilities can be distinguished by the value of $\operatorname{disc} n\left(V_{k}\right)$ in $k^{*} /\left(k^{*}\right)^{2}$ for any field $k$ in which 2 is not a square, such as $\mathbb{F}_{3}$ or $\mathbb{F}_{5}$. Such discriminants can be evaluated by a computer, as shown to the second author by Anton Leyton and Sabrina Pauli.

\subsection{Refined Euler classes and numbers}

Definition 5.13 (refined Euler class). Let $E \in \mathcal{S H}(S)$ be a homotopy ring spectrum, set $X \in \mathrm{Sch}_{S}$, and let $V \rightarrow X$ be a vector bundle and $\sigma: X \rightarrow V$ a section with zero scheme $Z=Z(\sigma)$. We denote by $e(V, \sigma)=e(V, \sigma, E) \in E_{Z}^{V^{*}}(X)$ the class corresponding to the composite

$$
X / X \backslash Z \stackrel{\sigma}{\rightarrow} V /\left.V \backslash 0 \simeq \Sigma^{V^{*}} \mathbb{1} \stackrel{u}{\rightarrow} \Sigma^{V^{*}} E\right|_{X} \in \mathcal{S H}(X)
$$

(see Example 4.2).

Remark 5.14. It is clear by construction that refined Euler classes are stable under base change.

Remark 5.15. In [56, Definition 3.9] the authors define for an SL-oriented ring spectrum $E$ and a vector bundle $p: V \rightarrow X$ the canonical Thom class $t h(V) \in E_{0}^{p^{*} V}(V)$, which one can check coincides with $e\left(p^{*} V, \sigma_{0}\right)$, where $\sigma_{0}$ is the tautological section of $p^{*} V$. Since $e(V, \sigma)=\sigma^{*} e\left(p^{*} V, \sigma_{0}\right)$, we deduce that

$$
e(V, \sigma)=\sigma^{*} \operatorname{th}(V) \text {. }
$$

Lemma 5.16. The 'forgetting support' map $E_{Z}^{V^{*}}(X) \rightarrow E^{V^{*}}(X)$ sends $e(V, \sigma)$ to $e(V)$. 
Proof. This follows from the commutative diagram

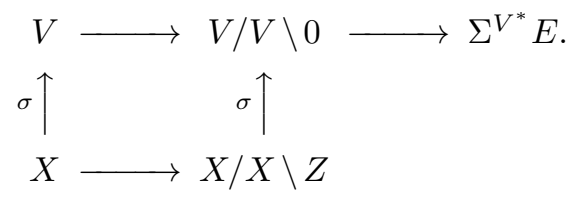

Indeed the composite from the bottom left to the top right along the top left represents $e(V)$, by Definition 5.1 (note that $\sigma$ is a homotopy inverse to the projection $V \rightarrow X$ ), whereas the composite along the bottom right represents $e(V, \sigma)$ with support forgotten, by Definition 5.13 and Example 4.2.

Definition 5.17 (refined Euler number). Suppose that $\pi: X \rightarrow S$ is smoothable lci, $Z \rightarrow S$ is finite, and $V$ is relatively oriented (so in particular, $r k(V)=r k\left(\mathrm{~L}_{\pi}\right)$ ). Suppose further that $E$ is SL-oriented. Then we put

$$
n(V, \sigma, \rho)=n(V, \sigma, \rho, E)=\pi_{*} e(V, \sigma) \in E^{0}(S) .
$$

Corollary 5.18. Suppose that additionally $\pi: X \rightarrow S$ is smooth and proper. Then $n(V, \sigma, \rho)=n(V, \rho) \in E^{0}(S)$.

Proof. Combine Lemma 5.16 and Example 4.7.

\subsection{Refined Euler classes and the six-functors formalism}

We now relate our Euler classes to the six-functors formalism. The following result shows that our refined Euler class coincides with the one defined by Déglise, Jin, and Khan [26, Remark 3.2.10]:

Proposition 5.19. Let $E \in \mathcal{S H}(S)$ be a homotopy ring spectrum, set $X \in \mathrm{Sm}_{S}$, and let $V$ be a vector bundle over $X$ and $\sigma$ a section of $V$. Then

$$
e(V, \sigma, E)=\sigma^{*} z_{*}(1),
$$

where $z: X \rightarrow V$ is the zero section and we use the canonical isomorphism $N_{z} \simeq V$ to form the push-forward $z_{*}$.

Proof. Let $p: V \rightarrow X$ be the projection and $s_{0}: V \rightarrow p^{*} V$ the canonical section. Then $\sigma^{*}\left(p^{*} V, \sigma_{0}\right)=(V, s)$, and hence, using Remark 5.14, it suffices to show that $z_{*}(1)=$ $e\left(p^{*} V, \sigma_{0}, E\right)$. By [31, last sentence of $\left.\S 2.1 .1\right]$, we know that

$$
z_{*}: E^{0}(X) \simeq E^{z^{*} p^{*} V^{*}+L_{z}} \rightarrow E_{X}^{p^{*} V^{*}}(V)
$$

is the purity equivalence. In the case of the zero section of a vector bundle, it just takes the tautological form

$$
[\mathbb{1}, E]_{X} \simeq[V / V \backslash 0, V / V \backslash 0 \wedge E]_{X} \simeq\left[V / V \backslash 0, p^{*} V / p^{*} V \backslash 0 \wedge E_{V}\right]_{V},
$$

and hence indeed sends 1 to $e\left(p^{*} V, \sigma_{0}\right)$.

It follows from the foregoing that Euler classes of vector bundles are determined by Euler classes of vector bundles with nondegenerate sections. 
Example 5.20. Let $V \rightarrow X$ be a vector bundle and $\sigma$ a section. Suppose $1 / 2 \in S$. The Euler class of $V$ in $\mathrm{KW}=\mathrm{KO}\left[\eta^{-1}\right]$ is given by the Koszul complex with its canonical symmetric bilinear form. Indeed, we may assume that $V$ has a regular section, in which case this follows from the existence of the morphism of cohomology theories BL ${ }^{\text {naive }} \rightarrow \mathrm{KW}$ and Proposition 2.11.

We deduce that Meta-Theorem 3.9 holds in this setting, even in a slightly stronger form with supports:

Corollary 5.21. If $\sigma$ is a nondegenerate section (i.e., locally given by a regular sequence), then $i_{*}(1)=e(V, \sigma) \in E_{Z}^{V^{*}}(X)$. In particular, forgetting supports (taking the image along $\left.E_{Z}^{V^{*}}(X) \rightarrow E^{V^{*}}(X)\right)$ we have $i_{*}(1)=e(V) \in E^{V^{*}}(X)$-that is, Meta-Theorem 3.9 holds in this situation.

Proof. The second statement follows from the first and Lemma 5.16; hence we shall prove the first. Consider the following cartesian square:

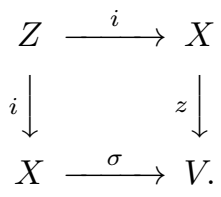

A well-known consequence of regularity of $\sigma$ is that this square is tor-independent. ${ }^{8}$ Since $i_{*}$ is compatible with tor-independent base change (Lemma 4.6(2)), we deduce from Proposition 5.19 that

$$
e(V, \sigma, E)=\sigma^{*} z_{*}(1)=i_{*} i^{*}(1)=i_{*}(1) .
$$

This was to be shown.

It follows that, as explained in $§ 3.4$, both the ordinary and refined Euler numbers in $E$-cohomology can be computed as sums of local indices. The remainder of this paper is mainly concerned with determining these indices, for certain examples of $E$.

Remark 5.22. These results can be generalized slightly. Fix a scheme $S$ and an SLoriented ring spectrum $E \in \mathcal{S H}(S)$.

(1) Let $X / S$ be smoothable lci and $V / X$ a vector bundle, relatively oriented in the sense of Definition 1.5. If $X / S$ is in addition proper, then we can transfer the Euler class along the structure morphism (see §4.2.4) as before to obtain an Euler number $n(V, \rho, E) \in E^{0}(S)$. More generally, without assuming $X / S$ proper, given a section $\sigma$ with zero scheme $Z$ proper over $S$, we obtain the refined Euler number $n(V, \sigma, \rho, E)$.

(2) Let $X / S$ be arbitrary, $V$ a vector bundle, and $\sigma$ a section of $V$ with zero scheme $i: Z \rightarrow X$, and suppose that $i$ is a regular immersion (but $\sigma$ need not be a

\footnotetext{
${ }^{8}$ To see this, note that $z_{*} \mathcal{O}_{X}$ can be resolved by the Koszul complex $K\left(p^{*} V, \sigma_{0}\right)$ for the tautological section $\sigma_{0}$ of the pullback $p^{*} V$ of $V$ along $p: V \rightarrow X$. It follows now from the projection formula that $z_{*} \mathcal{O}_{X} \otimes^{L} \sigma_{*} \mathcal{O}_{X} \simeq \sigma_{*} \sigma^{*} z_{*} \mathcal{O}_{X} \simeq \sigma_{*} \sigma^{*} K\left(p^{*} V, \sigma_{0}\right) \simeq \sigma_{*} K(V, \sigma)$. Since by definition $\sigma$ locally corresponds to a regular sequence (and $\sigma$ is affine), the claim follows.
} 
nondegenerate section-i.e., $Z$ could have higher dimension than expected). In this case there is an excess bundle $\mathcal{E}=\operatorname{cok}\left(\left.N_{Z / X} \rightarrow V\right|_{Z}\right) \cdot{ }^{9}$ A straightforward adaptation of the proof of Corollary 5.21, using the excess intersection formula [26, Proposition 3.3.4], shows that

$$
e(V, \sigma, E)=i_{*}(e(\mathcal{E}, E)) .
$$

(3) Putting everything together, let $X / S$ be proper smoothable lci, $V$ a relatively oriented vector bundle, and $\sigma$ a section with zero scheme $Z$ regularly immersed in $X$. Then

$$
n(V, \rho, E)=\sum_{Z^{\prime} \subset Z} n\left(\left.\mathcal{E}\right|_{Z^{\prime}}, \rho^{\prime}, E\right) .
$$

Here the sum is over clopen components $Z^{\prime}$ of $Z$, and $\rho^{\prime}$ denotes the induced relative orientation of $\mathcal{E}$. Note that if $\sigma$ is nondegenerate on $Z^{\prime}$-that is, $\left.\mathcal{E}\right|_{Z^{\prime}}=0$-then $e\left(\left.\mathcal{E}\right|_{Z^{\prime}}, E\right)=1 \in E^{0}\left(Z^{\prime}\right)$ and $n\left(\left.\mathcal{E}\right|_{Z^{\prime}}, \rho^{\prime}, E\right)=\operatorname{ind}_{Z^{\prime}}(\sigma)$ as before.

\section{6. $d$-Dimensional planes on complete intersections in projective space}

\subsection{Some Euler numbers of symmetric powers on Grassmannians}

Grassmannians and flag varieties are smooth and proper over $\mathbb{Z}$, and the Euler classes of many of their vector bundles have interesting interpretations in enumerative geometry. Computations over $\mathbb{R}$ and $\mathbb{C}$ are available in the literature in connection with enumerative results or accessible with localization techniques in equivariant cohomology [4]. The integrality of Euler classes, as in Theorem 5.11 and Proposition 5.4, can leverage such results to all fields. We do this now using the $\mathbb{Z}[1 / 2]$ case of Theorem 5.11 and a characteristic class argument. This is independent of recent work [18] on Hermitian $K$ theory over $\mathbb{Z}$, and the characteristic class argument may be of some independent interest. One can alternatively deduce Corollary 6.3 from the $\mathbb{Z}$ case of Theorem 5.11 and [18].

Remark 6.1. Suppose $X$ is a smooth, proper $\mathbb{Z}$-scheme with geometrically connected fibers and $\operatorname{Pic} X$ torsion free-for example, $X$ a Grassmannian or projective space. For a relatively orientable vector bundle $V \rightarrow X$ defined over $\mathbb{Z}$, there are at most two isomorphism classes of relative orientations. Namely, by assumption, there is a line bundle $L \rightarrow X$ and an isomorphism $\rho: L^{\otimes 2} \stackrel{\cong}{\longrightarrow} \omega_{X / \mathbb{Z}} \otimes \operatorname{det} V$. Since $\operatorname{Pic} X$ is torsion free, any relative orientation is an isomorphism $L^{\otimes 2} \cong \omega_{X / \mathbb{Z}} \otimes \operatorname{det} V$, whence two such differ by a global section of $\operatorname{Hom}\left(L^{\otimes 2}, L^{\otimes 2}\right) \cong \mathcal{O}(X)^{*}$. By hypothesis on $X$, the fibers of the push-forward of $\mathcal{O}_{X}$ all have rank 1, whence this push-forward is $\mathcal{O}_{\mathbb{Z}}$ and $\mathcal{O}(X)^{*} \cong \mathbb{Z}^{*}=\{ \pm 1\}$. Thus any relative orientation is isomorphic to $\rho$ or $-\rho$. We then have $n(V, \rho)=\langle-1\rangle n(V,-\rho)$.

Consequently we suppress the choice of orientation and just write $n(V)$. Beware that this does not mean that every vector bundle is relatively orientable, though!

\footnotetext{
${ }^{9}$ The map $\left.N_{Z / X} \rightarrow V\right|_{Z}$ is always injective, and is an isomorphism precisely if the section is nondegenerate.
} 
Remark 6.2. There is a canonical relative orientation for certain classes of vector bundles on Grassmannians $X=\operatorname{Gr}(d, n)$ : a point $p$ of $X$ with residue field $L$ corresponds to a dimension $d+1$ subspace of $L^{n+1}$. A choice of basis $\left\{e_{0}, \ldots, e_{n}\right\}$ of $L^{n+1}$ such that the span of $\left\{e_{0}, \ldots, e_{d}\right\}$ is $p$ defines canonical local coordinates for an affine chart isomorphic to $\mathbb{A}^{(n-d)(d+1)}$ with $p$ as the origin (see, e.g., [49, Definition 42]). This defines local trivializations of the tautological and quotient bundles on $X$, and therefore also of their tensor, symmetric, exterior powers, and their duals. A vector bundle $V$ formed from such operations on the tautological and quotient bundles and which is relatively orientable on $X$ inherits a canonical relative orientation $\rho$ such that the local coordinates and trivializations just described are compatible with $\rho$ in the sense of [49, Definition 21]. This is described in [49, Proposition 45] in a special case, but the argument holds in the stated generality. (One only needs the determinants of the clutching functions to be squares, which follows from the relative orientability of $V$. Together with the explicit coordinates, this gives the relative orienation.) This relative orientation has the property that it is defined over $\mathbb{Z}$, and for any very nondegenerate section $\sigma$, the data just described give a system of coordinates in the sense of Definition 2.28.

Corollary 6.3. Let $d \leq n$ be positive integers, and let $X=\operatorname{Gr}(d, n)$ be the Grassmannian of d-planes $\mathbb{P}^{d}$ in $\mathbb{P}^{n}$. Let $V=\oplus_{i=1}^{j} \operatorname{Sym}^{n_{i}} \mathcal{S}^{*}$, where $\mathcal{S}$ denotes the tautological bundle on $X$ and $n_{1}, \ldots, n_{j}$ are positive integers such that $\operatorname{rank} V=\operatorname{dim} X$ and $V$ is relatively orientable-that is, such that $\sum_{i=1}^{j}\left(\begin{array}{c}n_{i}+d \\ d\end{array}\right)=(d+1)(n-d)$ and $\sum_{i=1}^{j} \frac{n_{i}}{d+1}\left(\begin{array}{c}n_{i}+d \\ d\end{array}\right)+n+1$ is even. Then

$$
n(V)=\frac{n_{\mathbb{C}}+n_{\mathbb{R}}}{2}+\frac{n_{\mathbb{C}}-n_{\mathbb{R}}}{2}\langle-1\rangle
$$

over any ring in which 2 is invertible (where we interpret $n(V)$ as $n(V, K O)$ ) or any field (where we interpret $n(V)$ as $n^{\mathrm{GS}}(V)$ ).

Proof. Let $\mathcal{O}(1)$ denote the generator of $\operatorname{Pic} X$ given by the pullback of the tautological bundle under the Plücker embedding. Then $\omega_{X / \mathbb{Z}} \cong \mathcal{O}(-n-1)$ and $\operatorname{det} \operatorname{Sym}^{n_{i}} \mathcal{S}^{*} \cong$ $\mathcal{O}\left(\frac{n_{i}}{d+1}\left(\begin{array}{c}n_{i}+d \\ d\end{array}\right)\right)$. Thus $V$ is relatively orientable as a bundle over $\mathbb{Z}$, and Remarks 6.1 and 6.2 apply.

By the $\mathbb{Z}[1 / 2]$ case of Theorem 5.11 , it is enough to show that the discriminant of $n\left(V_{\mathbb{F}_{p}}\right)$ is trivial for some prime $p$ congruent to $1 \bmod 4$ and such that 2 is not a square (see Remark 5.12). Let EM(W) denote the Eilenberg-MacLane spectrum of the $\eta$-inverted Milnor-Witt sheaves $\mathrm{K}_{*}^{\mathrm{MW}}\left[\eta^{-1}\right]$ (compare [54, Remark 3.1]), and consider the associated Euler class $e(V, \operatorname{EM}(\mathrm{W}))$. Then $n(V, \operatorname{EM}(\mathrm{W}))$ determines the Witt class of $n(V)$, and hence the discriminant of $n(V)$ as well.

$d$ is even: Suppose that $d$ is even. Let $\pi$ denote the structure map of $X$. Since $n(V, \operatorname{EM}(\mathrm{W}))=\pi_{*} \prod_{i=1}^{j} e\left(\operatorname{Sym}^{n_{i}} \mathcal{S}^{*}, \operatorname{EM}(\mathrm{W})\right)$, it is enough to show that $e\left(\operatorname{Sym}^{n_{1}} \mathcal{S}^{*}, \operatorname{EM}(\mathrm{W})\right)=0$. By the Jouanolou device, we may assume that any vector bundle is pulled back from the universal bundle. It is therefore enough to show the same for the dual tautological bundle on the universal Grassmannian $B \mathrm{GL}_{d+1}$-that is, let $\mathcal{S}_{d+1}^{*}$ denote the dual of the tautological bundle on $B \mathrm{GL}_{d+1}$; we show that $e\left(\operatorname{Sym}^{n_{1}} \mathcal{S}_{d+1}^{*}, \operatorname{EM}(\mathrm{W})\right)=0$. By Ananyevskiy's splitting principle [2, Theorem 6] and 
its extension due to M. Levine [54, Theorem 4.1], we may show the vanishing of the $\mathrm{EM}(\mathrm{W})$-Euler class of $\mathrm{Sym}^{n_{1}} \mathcal{S}_{d+1}^{*}$ after pullback to

$$
B \mathrm{SL}_{2} \times B \mathrm{SL}_{2} \times \cdots \times B \mathrm{SL}_{2} \times B \mathrm{SL}_{1}
$$

via the map classifying the external Whitney sum of the tautological bundles. Here we use the fact that $d+1$ is odd. This pullback of $\operatorname{Sym}^{n_{1}} \mathcal{S}_{d+1}^{*}$ contains the odd-rank summand $\operatorname{Sym}^{n_{1}} \mathcal{S}_{1}^{*}$, and therefore its $\operatorname{EM}(\mathrm{W})$-Euler class is 0 as desired (see [2, Lemma 3] and [54, Lemma 4.3]).

$d$ is odd: Let $k$ be a finite field whose order is prime to $2 \prod_{i=1}^{j}\left(n_{i}\right)$ !, congruent to 1 $\bmod 4$ (so -1 is a square), and such that 2 is not a square. By Theorem 5.11 , it suffices to show that the discriminant of $n(V, \operatorname{Gr}(d, n)) \in \mathrm{W}(k) \cong \mathrm{GW}(k) / \mathbb{Z} h$ is trivial (compare Remark 5.12).

Define $r$ in $\mathbb{Z}$ such that $d=2 r+1$. Let $S_{d+1}^{*}$ denote the dual tautological bundle on $B \mathrm{SL}_{d+1}$ and let $p_{1}, \ldots, p_{r}, p_{r+1}$ and $e$ in $\operatorname{EM}(\mathrm{W})^{*}\left(B \mathrm{SL}_{d+1}\right)$ denote its Pontryagin and Euler classes, respectively. (Often one would let $p_{i}$ be the Pontryagin classes of the tautological bundle, not its dual, but this is more convenient here.) By Lemma 6.5, $e\left(\operatorname{Sym}^{n_{i}} S_{d+1}^{*}, \operatorname{EM}(\mathrm{W})\right)$ is in the image of $\mathbb{Z}\left[p_{1}, \ldots, p_{r}, e\right] \rightarrow \operatorname{EM}(\mathrm{W})^{*}\left(B \mathrm{SL}_{d+1}\right)$. (Note that we have omitted $p_{r+1}$, as $p_{r+1}=e^{2}$ [2, Corollary 3].) Therefore $e(V, \operatorname{Gr}(d, n))$ can be expressed as a polynomial with integer coefficients in the Pontryagin classes and Euler class of the dual tautological bundle $\mathcal{S}^{*}$ on $\operatorname{Gr}(d, n)$. By Lemma 6.7, it follows that $\operatorname{disc} n(V, \operatorname{Gr}(d, n))=1$ in $k^{*} /\left(k^{*}\right)^{2}$ as desired.

M. Levine [54] uses the normalizer $N$ of the standard torus of $\mathrm{SL}_{2}$,

$$
1 \rightarrow\left\{\left(\begin{array}{cc}
t & 0 \\
0 & t^{-1}
\end{array}\right)\right\} \rightarrow N \rightarrow\left\{\left(\begin{array}{cc}
0 & 1 \\
-1 & 0
\end{array}\right)\right\} \rightarrow 1
$$

and bundles $\tilde{O}(a)$ and $\tilde{O}^{-}(a)$ for $a$ in $\mathbb{Z}$ corresponding respectively to the representations

$$
\begin{gathered}
\left(\begin{array}{cc}
t & 0 \\
0 & t^{-1}
\end{array}\right) \mapsto\left(\begin{array}{cc}
t^{a} & 0 \\
0 & t^{-a}
\end{array}\right) \\
\left(\begin{array}{cc}
0 & 1 \\
-1 & 0
\end{array}\right) \mapsto\left(\begin{array}{cc}
0 & 1 \\
(-1)^{a} & 0
\end{array}\right)
\end{gathered}
$$

and

$$
\begin{gathered}
\left(\begin{array}{cc}
t & 0 \\
0 & t^{-1}
\end{array}\right) \mapsto\left(\begin{array}{cc}
t^{a} & 0 \\
0 & t^{-a}
\end{array}\right) \\
\left(\begin{array}{cc}
0 & 1 \\
-1 & 0
\end{array}\right) \mapsto\left(\begin{array}{cc}
0 & -1 \\
(-1)^{a+1} & 0
\end{array}\right),
\end{gathered}
$$

to compute characteristic classes, and we use his technique. We will use the notation $\tilde{O}^{(-)}(a)$ to mean either $\tilde{O}(a)$ or $\tilde{O}^{-}(a)$ when a claim holds for both possibilities. We likewise use the $\mathrm{EM}(\mathrm{W})$-Pontryagin (or Borel) classes of a vector bundle with trivialized determinant of Panin and Walter [64]. For background on these classes, see [2, Introduction, Section 3], [54, Section 3], or [74, Section 2]. 
Let $e_{i} \in \operatorname{EM}(\mathrm{W})^{*}\left(N^{r+1}\right)$ denote the pullback of $e\left(S_{2}^{*}, \operatorname{EM}(\mathrm{W})\right)$ under the $i$ th projection $B N^{r+1} \rightarrow B N$ composed with the canonical map $B N \rightarrow B \mathrm{SL}_{2}$.

Given vector bundles $V$ and $E$ on schemes $X$ and $Y$, respectively, let $V \otimes E$ denote the vector bundle on $X \times Y$ given by the tensor product of the pullbacks of $V$ and $E$.

Lemma 6.4. Suppose $r \geq 1$ and $a_{1}, \ldots, a_{r+1}$ are integers, and that our base scheme is a field $k$ with characteristic not dividing $2 \prod_{i=1}^{r+1} a_{i}$. The Euler class $e\left(\bigotimes_{i=1}^{r+1} \tilde{O}^{(-)}\left(a_{i}\right), \mathrm{EM}(\mathrm{W})\right)$ and $\mathrm{EM}(\mathrm{W})$-Pontryagin classes $p_{j}\left(\otimes_{i=1}^{r+1} \tilde{O}^{(-)}\left(a_{i}\right), \mathrm{EM}(\mathrm{W})\right)$ for $j=1, \ldots, 2^{r}$ are in the image of the map

$$
\mathbb{Z}\left[e_{1}^{2}, \ldots, e_{r+1}^{2}\right] \rightarrow \operatorname{EM}(\mathrm{W})^{*}\left(B N^{r+1}\right) .
$$

Proof. Proceed by induction on $r$. By Ananyevskiy's splitting principle [2, Theorem 6], there exists a map $\pi: Y \rightarrow B N^{r}$ such that the pullback of $\bigotimes_{i=2}^{r+1} \tilde{O}^{(-)}\left(a_{i}\right)$ is a direct sum of rank 2 bundles $V_{i}$ on $Y$,

$$
\pi^{*} \bigotimes_{i=2}^{r+1} \tilde{O}^{(-)}\left(a_{i}\right) \cong \oplus_{i=1}^{2^{r-1}} V_{i}
$$

and the map

$$
\operatorname{EM}(\mathrm{W})^{*}\left(B N \times B N^{r}\right) \rightarrow \operatorname{EM}(\mathrm{W})^{*}(B N \times Y)
$$

is injective. We pull back the vector bundle $\bigotimes_{i=1}^{r+1} \tilde{O}^{(-)}\left(a_{i}\right)$ along the map $1_{B N} \times \pi: B N \times$ $Y \rightarrow B N^{r+1}$ and obtain an isomorphism

$$
\left(1_{B N} \times \pi\right)^{*} \bigotimes_{i=1}^{r+1} \tilde{O}^{(-)}\left(a_{i}\right) \cong \oplus_{i=1}^{2^{r-1}}\left(\tilde{O}^{(-)}\left(a_{1}\right) \otimes V_{i}\right)
$$

By [54, Theorem 7.1] and the equality $e\left(S_{2}, \operatorname{EM}(\mathrm{W})\right)^{2}=e\left(S_{2}^{*}, \operatorname{EM}(\mathrm{W})\right)^{2}$, which follows from [55, Theorem 11.1],

$$
e\left(\tilde{O}^{(-)}\left(a_{1}\right), \operatorname{EM}(\mathrm{W})\right)^{2}=a_{1}^{2} e\left(S_{2}^{*}, \mathrm{EM}(\mathrm{W})\right)^{2}
$$

Since $\tilde{O}^{(-)}\left(a_{1}\right)$ and $V_{i}$ both have rank 2, [54, Proposition 9.1] and equation (9) imply that

$$
e\left(\tilde{O}^{(-)}\left(a_{1}\right) \otimes V_{i}, \mathrm{EM}(\mathrm{W})\right)=a_{1}^{2} e_{1}^{2}-e\left(V_{i}, \mathrm{EM}(\mathrm{W})\right)^{2}
$$

and

$$
p_{1}\left(\tilde{O}^{(-)}\left(a_{1}\right) \otimes V_{i}, \operatorname{EM}(\mathrm{W})\right)=2\left(a_{1}^{2} e_{1}^{2}+e\left(V_{i}, \operatorname{EM}(\mathrm{W})\right)^{2}\right) .
$$

This establishes the claim when $r=1$, because $p_{2}=e^{2}$.

We now assume the claim holds for $r-1$. By formula (8),

$$
e\left(\left(1_{B N} \times \pi\right)^{*} \otimes_{i=1}^{r+1} \tilde{O}^{(-)}\left(a_{i}\right), \operatorname{EM}(\mathrm{W})\right)=\prod_{i=1}^{2^{r-1}} e\left(\tilde{O}^{(-)}\left(a_{1}\right) \otimes V_{i}, \mathrm{EM}(\mathrm{W})\right)
$$


and

$$
p\left(\left(1_{B N} \times \pi\right)^{*} \bigotimes_{i=1}^{r+1} \tilde{O}^{(-)}\left(a_{i}\right), \operatorname{EM}(\mathrm{W})\right)=\prod_{i=1}^{2^{r-1}} p\left(\tilde{O}^{(-)}\left(a_{1}\right) \otimes V_{i}, \operatorname{EM}(\mathrm{W})\right),
$$

where $p$ denotes the total Pontryagin class.

Combining equations (10) and (12) shows that

$$
\begin{aligned}
e & \left(\left(1_{B N} \times \pi\right)^{*} \nabla_{i=1}^{r+1} \tilde{O}^{(-)}\left(a_{i}\right), \operatorname{EM}(\mathrm{W})\right) \\
& =\sum_{i=0}^{2^{r-1}}\left(a_{1} e_{1}\right)^{2 i} \sigma_{2^{r-1}-i}\left(e\left(V_{1}, \operatorname{EM}(\mathrm{W})\right)^{2}, \ldots, e\left(V_{2^{r-1}}, \operatorname{EM}(\mathrm{W})\right)^{2}\right)
\end{aligned}
$$

where $\sigma_{i}$ denotes the $i$ th elementary symmetric function. Since the $V_{i}$ have rank 2 ,

$$
e\left(V_{1}, \operatorname{EM}(\mathrm{W})\right)^{2}=p_{1}\left(V_{1}, \operatorname{EM}(\mathrm{W})\right),
$$

and the Whitney sum formula for Pontryagin classes implies that

$$
p_{i}\left(\oplus_{j=1}^{2^{r-1}} V_{i}, \operatorname{EM}(\mathrm{W})\right)=\sigma_{i}\left(e\left(V_{1}, \operatorname{EM}(\mathrm{W})\right)^{2}, \ldots, e\left(V_{2^{r-1}}, \operatorname{EM}(\mathrm{W})\right)^{2}\right) .
$$

Since $\oplus_{j=1}^{2^{r-1}} V_{i} \cong \pi^{*} \bigotimes_{i=2}^{r+1} \tilde{O}^{(-)}\left(a_{i}\right)$, it follows by induction that $e\left(\nabla_{i=1}^{r+1} \tilde{O}^{(-)}\left(a_{i}\right), \operatorname{EM}(\mathrm{W})\right)$ is in the image of $\mathbb{Z}\left[e_{1}^{2}, \ldots, e_{r+1}^{2}\right]$.

Combining equations (10), (11), and (13), we have

$$
\begin{aligned}
p & \left(\left(1_{B N} \times \pi\right)^{*} \bigotimes_{i=1}^{r+1} \tilde{O}^{(-)}\left(a_{i}\right), \operatorname{EM}(\mathrm{W})\right) \\
& =\prod_{i=1}^{2^{r-1}}\left(1+2\left(a_{1}^{2} e_{1}^{2}+e\left(V_{i}, \operatorname{EM}(\mathrm{W})\right)^{2}\right)+\left(a_{1}^{2} e_{1}^{2}-e\left(V_{i}, \operatorname{EM}(\mathrm{W})\right)^{2}\right)^{2}\right) .
\end{aligned}
$$

Because the elementary symmetric polynomials generate all symmetric polynomials, it follows that $p\left(\left(1_{B N} \times \pi\right)^{*} \bigotimes_{i=1}^{r+1} \tilde{O}^{(-)}\left(a_{i}\right), \operatorname{EM}(\mathrm{W})\right)$ is in the image of

$$
\mathbb{Z}\left[e_{1}^{2}, \sigma_{i}\left(e\left(V_{1}, \operatorname{EM}(\mathrm{W})\right)^{2}, \ldots, e\left(V_{2^{r-1}}, \operatorname{EM}(\mathrm{W})\right)^{2}\right): i=1, \ldots, 2^{r-1}\right] .
$$

As before, these elementary symmetric functions are the Pontryagin classes of the pullback of $\bigotimes_{i=2}^{r+1} \tilde{O}^{(-)}\left(a_{i}\right)$, finishing the proof by induction.

Let $p_{1}, \ldots, p_{r}$ and $e$ in $\operatorname{EM}(\mathrm{W})^{*}\left(B \mathrm{SL}_{d+1}\right)$ denote the Pontryagin and Euler classes, respectively, of the dual tautological bundle $S_{d+1}^{*}$ on $B \mathrm{SL}_{d+1}$.

Lemma 6.5. Let $d=2 r+1$ be an odd integer and let $n$ be a positive integer. Let $k$ be a field of characteristic not dividing $2 n$ !. Then $e\left(\operatorname{Sym}^{n} S_{d+1}^{*}, \operatorname{EM}(\mathrm{W})\right)$ is in the image of $\mathbb{Z}\left[p_{1}, \ldots, p_{r}, e\right] \rightarrow \operatorname{EM}(\mathrm{W})^{*}\left(B \mathrm{SL}_{d+1}\right)$.

Proof. Let $f$ denote the composite

$$
B N^{r+1} \rightarrow \mathrm{BSL}_{2}^{r+1} \rightarrow \mathrm{BSL}_{d+1}
$$


of the $(r+1)$-fold product of the canonical map $B N \rightarrow B \mathrm{SL}_{2}$ with the map $B \mathrm{SL}_{2}^{r+1} \rightarrow$ $B \mathrm{SL}_{d+1}$ classifying the external direct sum $\oplus_{i=1}^{r+1} S_{2}$. There is an isomorphism

$$
f^{*} \operatorname{Sym}^{n} S_{d+1}^{*} \cong \bigoplus_{\substack{\left(a_{1}, \ldots, a_{r+1}\right) \in \mathbb{Z}_{\geq 0}^{r+1} \\ \sum_{i} a_{i}=n}} \otimes_{i=1}^{r} \operatorname{Sym}^{a_{i}} \tilde{O}(1) .
$$

By inspection, the symmetric powers of the tautological bundle $\tilde{O}(1)$ on $B N$ split into a sum of bundles of rank $\leq 2$ (compare [54, p. 38]):

$$
\operatorname{Sym}^{a} \tilde{O}(1) \cong \begin{cases}\oplus_{l=0}^{b} \tilde{O}^{(-1)^{l}}(i-2 l) & \text { when } a=2 b+1 \text { is odd } \\ \oplus_{l=0}^{b-1} \tilde{O}^{(-1)^{l}}(i-2 l) \oplus \mathcal{O} & \text { when } a=2 b \text { is even and } b \text { is even } \\ \oplus_{l=0}^{b-1} \tilde{O}^{(-1)^{l}}(i-2 l) \oplus \gamma & \text { when } a=2 b \text { is even and } b \text { is odd }\end{cases}
$$

where $\gamma$ is the line bundle corresponding to the representation $N \rightarrow \mathrm{GL}_{1}$ sending the torus to 1 and $\left(\begin{array}{cc}0 & 1 \\ -1 & 0\end{array}\right)$ to -1 .

Combining formulas (14) and (15), we can decompose $f^{*} \operatorname{Sym}^{n} S_{d+1}^{*}$ into a direct sum with summands which have various numbers of factors of rank 2 . Separate these summands into those with at least two rank 2 factors and those with only one rank 2 factor, if any of the latter sort appear. (This occurs when we can take all but one $a_{i}$ to be even.) The direct sum of the latter such terms can alternatively be expressed as a sum of pullbacks of $\operatorname{Sym}^{a_{i}} \tilde{O}(1)$ under some projection $N^{r+1} \rightarrow N$ tensored with some $\gamma$ s pulled back from other projections. We may ignore the factors of $\gamma$ by $[55, \S 10$ p. 78 (2)], because $e(\gamma)=0$, as $\gamma$ is a bundle of odd rank. Since $\tilde{O}$ has rank 2 and the characteristic of $k$ does not divide $2 a_{i}$, we may apply [54, Theorem 8.1] and conclude that $e\left(\operatorname{Sym}^{a_{i}} \tilde{O}(1)\right)$ is an integer multiple of a power of $e(\tilde{O}(1))$. Since the summands are symmetric under the permutation action of the symmetric group on $r+1$ letters on $B N^{r+1}$, it follows that the Euler class of these summands is an integer multiple of a power of $e$.

We now consider the Euler class of the rest of the summands. It suffices to show that the Euler class $\epsilon_{1}$ of the summands with at least two rank 2 factors is also in the image of $\mathbb{Z}\left[p_{1}, \ldots, p_{r}, e\right]$. We may again ignore the factors of $\gamma$, as these do not change the Euler class. By Lemma $6.4, \epsilon_{1}$ is the image of an element of $\mathbb{Z}\left[e_{1}^{2}, \ldots, e_{r+1}^{2}\right]$. Moreover, because each tuple $\left(a_{1}, \ldots, a_{r+1}\right)$ of the direct sum occurs in every permutation, we may choose an element of $\mathbb{Z}\left[e_{1}^{2}, \ldots, e_{r+1}^{2}\right]$ which is invariant under the permutation action of the symmetric group on $r+1$ letters and which maps to $\epsilon_{1}$. Thus, $\epsilon_{1}$ is in the image of the map

$$
\mathbb{Z}\left[\sigma_{1}\left(e_{1}^{2}, \ldots, e_{r+1}^{2}\right), \ldots, \sigma_{r+1}\left(e_{1}^{2}, \ldots, e_{r+1}^{2}\right)\right] \rightarrow \operatorname{EM}(\mathrm{W})^{*}\left(B N^{r+1}\right),
$$

where $\sigma_{i}$ denotes the $i$ th elementary symmetric polynomial. Since $\sigma_{i}\left(\left(e_{1}^{2}, \ldots, e_{r+1}^{2}\right)\right)$ is the pullback to $B N$ of $p_{i}\left(S_{d+1}^{*} \rightarrow B \mathrm{SL}_{d+1}, \operatorname{EM}(\mathrm{W})\right)$, we have that $\epsilon_{1}$ is in the image of $\mathbb{Z}\left[p_{1}, \ldots, p_{r}, e\right]$, as desired. 
The Pontryagin and Euler classes of $\mathcal{S}_{d+1}^{*} \rightarrow \operatorname{Gr}(d, n)$ are pulled back from those of $\mathcal{S}_{d+1}^{*} \rightarrow B \mathrm{GL}_{d+1}$. The $\mathrm{EM}(\mathrm{W})^{*}$-cohomology and twisted cohomology of $B \mathrm{GL}_{d+1}$ inject into that of $B \mathrm{SL}_{d+1}$,

$$
\operatorname{EM}(\mathrm{W})^{*}\left(B \mathrm{GL}_{d+1}\right) \oplus \operatorname{EM}(\mathrm{W})^{*}\left(B \mathrm{GL}_{d+1}, \operatorname{det} \mathcal{S}\right) \subseteq \operatorname{EM}(\mathrm{W})^{*}\left(B \mathrm{SL}_{d+1}\right),
$$

by [54, Theorem 4.1]. Under this injection, the Pontrygin and Euler classes of $\mathcal{S}_{d+1}^{*} \rightarrow$ $B \mathrm{GL}_{d+1}$ are sent to $p_{1}, \ldots, p_{r}, p_{r+1}$ and to $e$, respectively, so we will let $p_{i}$ and $e$ denote the corresponding characteristic classes of $\mathcal{S}^{*} \rightarrow B \mathrm{GL}_{d+1}$ and $\mathcal{S}_{d+1}^{*} \rightarrow \operatorname{Gr}(d, n)$ as well. Let $\pi: \operatorname{Gr}(d, n) \rightarrow$ Spec $k$ denote the structure map and

$$
\pi_{*}: \operatorname{EM}(\mathrm{W})^{*}\left(\operatorname{Gr}(d, n),\left(\operatorname{det} \mathcal{S}_{d+1}^{*}\right)^{\otimes-(n+1)}\right) \rightarrow \mathrm{W}(k)
$$

the induced push-forward on $\operatorname{EM}(\mathrm{W})^{*}$-cohomology.

Lemma 6.6. Let $d=2 r+1$ be odd. For any nonnegative integers $a_{1}, \ldots, a_{r+1}, b$ such that $\sum(4 i) a_{i}+b(d+1)=(d+1)(n-d)$ and $b \equiv n+1 \bmod 2$, the monomial $e^{b} \prod_{i=1}^{r+1} p_{i}^{a_{i}}$ in $\operatorname{EM}(\mathrm{W})^{*}\left(\operatorname{Gr}(d, n),\left(\operatorname{det} \mathcal{S}_{d+1}^{*}\right)^{\otimes b}\right)$ is in the image of $\mathbb{Z}[e]-$ or in other words, there exists c in $\mathbb{Z}$ such that $e^{b} \prod_{i=1}^{r+1} p_{i}^{a_{i}}=c e^{n-d}$.

Proof. Let $Q$ denote the quotient bundle on $\operatorname{Gr}(d, n)$, defined by the short exact sequence

$$
0 \rightarrow \mathcal{S}_{d+1} \rightarrow \mathcal{O}^{n+1} \rightarrow Q \rightarrow 0 .
$$

In particular, the rank of $Q$ is $n-d$. The nonvanishing Pontryagin classes of $\mathcal{S}_{d+1}$ are $p_{1}, \ldots, p_{r}, p_{r+1}$, with $e^{2}=p_{r+1}$. Define $s$ so that $n-d=2 s$ or $n-d=2 s+1$, depending on whether $n-d$ is odd or even. Let $p_{1}^{\perp}, \ldots, p_{s}^{\perp}$ denote the nonvanishing Pontryagin classes of the dual to the quotient bundle $Q^{*}$ on $\operatorname{Gr}(d, n)$. By [2, Lemma 15],

$$
\left(1+p_{1}+\cdots+p_{r+1}\right)\left(1+p_{1}^{\perp}+\cdots+p_{s}^{\perp}\right)=1
$$

in $W^{*}(\operatorname{Gr}(d, n))$. Setting the notation $A$ for the ring

$$
A=\mathbb{Z}\left[p_{1}, \ldots, p_{r}, p_{r+1}, p_{1}^{\perp}, \ldots, p_{s}^{\perp}\right] /\left\langle\left(1+p_{1}+\cdots+p_{r}+p_{r+1}\right)\left(1+p_{1}^{\perp}+\cdots+p_{s}^{\perp}\right)-1\right\rangle,
$$

we therefore have a homomorphism $\tau: A \rightarrow \operatorname{EM}(\mathrm{W})^{*}(\operatorname{Gr}(d, n)$. There is a canonical isomorphism

$$
\mathrm{H}^{*}(\mathbb{C} \mathrm{Gr}(r, r+s) ; \mathbb{Z}) \cong A,
$$

where $\mathrm{H}^{*}(\mathbb{C} \operatorname{Gr}(r, r+s) ; \mathbb{Z})$ denotes the singular cohomology of the $\mathbb{C}$-manifold associated to the $\mathbb{C}$-points of the Grassmannian $\operatorname{Gr}(r, r+s)$, sending the $i$ th Chern class of the dual tautological bundle to $p_{i}$. The top-dimensional singular cohomology $\mathrm{H}^{(r+1) s}(\mathbb{C} \mathrm{Gr}(r, r+$ $s) ; \mathbb{Z})$ is isomorphic to $\mathbb{Z}$ by Poincaré duality. Under our chosen isomorphism, the monomial $p_{r+1}^{s}$ corresponds to the top Chern class $c_{(r+1) s}\left(\mathcal{S}_{r+1}^{\oplus s} \rightarrow \mathbb{C} \operatorname{Gr}(r, r+s)\right)$ of the direct sum of $s$-copies of the dual tautological bundle, which is a generator (with the usual $\mathbb{C}$-orientations, $c_{(r+1) s}\left(\mathcal{S}_{r+1}^{\oplus s} \rightarrow \mathbb{C G r}(r, r+s)\right)$ counts the number of linear subspaces of dimension $r$ in a complete intersection of $s$ linear hypersurfaces in $\mathbb{C P}^{r+s}$, and this number is 1 ; compare Remark 6.9 and Lemma 6.7). Therefore, for any monomial $\prod_{i=1}^{r+1} p_{i}^{a_{i}^{\prime}}$ with 
$\sum_{i=1}^{r+1} i a_{i}^{\prime}=(r+1) s$, there is an integer $c^{\prime}$ such that

$$
\prod_{i=1}^{r+1} p_{i}^{a_{i}^{\prime}}=c^{\prime} p_{r+1}^{s}
$$

in $\mathrm{H}^{*}(\mathbb{C} \operatorname{Gr}(r, r+s) ; \mathbb{Z})$.

Since $d$ is odd, $n+1 \equiv n-d \bmod 2$, and therefore $b \equiv n-d \bmod 2$. Note that if $n-d$ is odd, $b \geq 0$. We may then define a nonnegative integer $b^{\prime}$ by the rule

$$
b^{\prime}= \begin{cases}b / 2 & \text { if } n-d \equiv 0 \bmod 2, \\ (b-1) / 2 & \text { if } n-d \equiv 1 \bmod 2 .\end{cases}
$$

With this notation, $n-d-b=2\left(s-b^{\prime}\right)$. Thus

$$
\sum(4 i) a_{i}=(d+1)(n-d-b)=(d+1) 2\left(s-b^{\prime}\right)=4(r+1)\left(s-b^{\prime}\right),
$$

whence

$$
\sum i a_{i}=(r+1)\left(s-b^{\prime}\right)
$$

By equation (16), there is an integer $c$ such that we have the equality

$$
p_{r+1}^{b^{\prime}} \prod_{i=1}^{r+1} p_{i}^{a_{i}}=c p_{r+1}^{s}
$$

in $\mathrm{H}^{*}(\mathbb{C} \operatorname{Gr}(r, r+s) ; \mathbb{Z})$. Applying $\tau$, we see that

$$
e^{2 b^{\prime}} \prod_{i=1}^{r+1} p_{i}^{a_{i}}=c^{\prime} e^{2 s}
$$

which implies the claim, either immediately if $n-d$ is even or by multiplying by $e$ if $n-d$ is odd.

Lemma 6.7. Let $d$ be odd. Suppose $k$ is a finite field such that -1 is a square. For any nonnegative integers $a_{1}, \ldots, a_{r+1}, b$ such that $b(d+1)+\sum_{i=1}^{r+1} 4 i a_{i}=(d+1)(n-d)$ and $b \equiv n+1 \bmod 2$, the push-forward $\pi_{*}\left(e^{b} \prod_{i=1}^{r+1} p_{i}^{a_{i}}\right)$ has trivial discriminant.

Remark 6.8. The condition $b \equiv n+1 \quad \bmod 2$ ensures that $e^{b} \prod_{i=1}^{r+1} p_{i}^{a_{i}}$ lies in the appropriate twist of the Witt cohomology of $\operatorname{Gr}(d, n)$-that is, $e^{b} \prod_{i=1}^{r+1} p_{i}^{a_{i}}$ is in $\operatorname{EM}(\mathrm{W})^{*}\left(\operatorname{Gr}(d, n), \omega_{\mathrm{Gr}(d, n)} / k\right)$ as opposed to $\operatorname{EM}(\mathrm{W})^{*}(\operatorname{Gr}(d, n))$, so that we may apply $\pi_{*}$. The condition on the sum $b(d+1)+\sum_{i=1}^{r+1} 4 i a_{i}$ ensures that $e^{b} \prod_{i=1}^{r+1} p_{i}^{a_{i}}$ lies in the $(d+1)(n-d)$-degree $\operatorname{EM}(\mathrm{W})^{*}$-cohomology of $\operatorname{Gr}(d, n)$, so the codomain of $\pi_{*}$ is $\mathrm{W}(k)$.

Proof. By Lemma 6.6, it suffices to show that $\operatorname{disc} \pi_{*} e^{n-d}=1$. We may identify $\pi_{*} e^{n-d}$ with the Euler number of $\oplus_{j=1}^{n-d} \mathcal{S}^{*}$ :

$$
\pi_{*} e^{n-d}=n\left(\oplus_{j=1}^{n-d} \mathcal{S}^{*}, \operatorname{EM}(\mathrm{W})\right)
$$


Let $x_{0}, \ldots, x_{n}$ be coordinates on a projective space $\mathbb{P}_{k}^{n}=\operatorname{Proj} k\left[x_{0}, \ldots, x_{n}\right]$. The Euler number $n\left(\oplus_{j=1}^{n-d} \mathcal{S}^{*}, \operatorname{EM}(\mathrm{W})\right)$ can be calculated with the section $\sigma=\oplus_{i=d+1}^{n} x_{i}$ as in $\S 2.4$. There is an analogous section $\sigma_{\mathbb{Z}}=\oplus_{i=d+1}^{n} x_{i}$ of $\oplus_{j=1}^{n-d} \mathcal{S}_{\mathbb{Z}}^{*} \rightarrow \operatorname{Gr}(d, n)_{\mathbb{Z}}$ defined over $\mathbb{Z}$. The zero locus $\sigma_{\mathbb{Z}}=0$ is the single $\mathbb{Z}$-point of the Grassmannian associated to the linear subspace of $\mathbb{P}_{k}^{n}$ given by $x_{d+1}=x_{d+2}=\cdots=x_{n}=0$.

The vanishing locus of $\sigma_{\mathbb{Z}}$ is the origin of the affine space $\mathbb{A}_{\mathbb{Z}}^{(d+1)(n-d)}=$ $\operatorname{Spec} \mathbb{Z}\left[a_{i j}: i=0, \ldots, d, j=d+1, \ldots, n\right] \hookrightarrow \operatorname{Gr}(d, n)$ whose point $\left(a_{i j}\right)$ corresponds to the row space of

$$
\left(\begin{array}{cccccccc}
1 & 0 & \cdots & 0 & a_{0, d+1} & a_{0, d+2} & \cdots & a_{0, n} \\
0 & 1 & \cdots & 0 & a_{1, d+1} & a_{1, d+2} & \cdots & a_{1, n} \\
\vdots & \vdots & \ddots & \vdots & \vdots & \vdots & \ddots & \vdots \\
0 & 0 & \cdots & 1 & a_{d, d+1} & a_{d, d+2} & \cdots & a_{d, n}
\end{array}\right) .
$$

For a description of these coordinates on this open affine of the Grassmannian, see, for example, [28, Section 3.2]. Let $e_{0}, \ldots, e_{n}$ denote the standard basis of the free module of rank $n+1$. Let $\tilde{e}_{0}, \ldots, \tilde{e}_{n}$ denote the basis consisting of the row space of matrix (17) followed by $e_{d+1}, e_{d+1}, \ldots, e_{n}$. Let $\tilde{x}_{0}, \ldots, \tilde{x}_{n}$ denote the dual basis to $\tilde{e}_{0}, \ldots, \tilde{e}_{n}$. Over this $\mathbb{A}_{\mathbb{Z}}^{(d+1)(n-d)}$, the vector bundle $\mathcal{S}_{\mathbb{Z}}^{*}$ is trivialized by the basis of sections $\left\{\tilde{x}_{0}, \ldots, \tilde{x}_{d}\right\}$. Then we may interpret $\sigma$ as a function $\mathbb{A}_{\mathbb{Z}}^{(d+1)(n-d)} \rightarrow \mathbb{A}_{\mathbb{Z}}^{(d+1)(n-d)}$. Namely,

$$
x_{u}\left(\left(a_{i j}\right)\right)=\sum_{l=0}^{d}\left(x_{u}\left(a_{i j}\right)\right)\left(\tilde{e}_{l}\right) \tilde{x}_{l}
$$

for

$$
u=d+1, \ldots, n \text {. }
$$

As a subscheme, $\sigma_{\mathbb{Z}}=0$ is therefore the zero locus of

$$
\left(x_{u}\left(a_{i j}\right)\right)\left(\tilde{e_{l}}\right)=a_{l, u}
$$

for $l=0, \ldots, d$ and $u=d+1, \ldots, n$. Thus the subscheme of $\operatorname{Gr}(d, n)_{\mathbb{Z}}$ given by $\left\{\sigma_{\mathbb{Z}}=0\right\}$ is a section of the structure map $\operatorname{Gr}(d, n)_{\mathbb{Z}} \rightarrow \operatorname{Spec} \mathbb{Z}$. In particular, it is finite and étale of rank 1. It follows that the Jacobian of $\sigma$,

$$
\operatorname{Jac} \sigma \in \operatorname{Hom}\left(\left.\operatorname{det} T \operatorname{Gr}(d, n)\right|_{\left\{\sigma_{\mathbb{Z}}=0\right\}},\left.\operatorname{det} \oplus_{j=1}^{n-d} \mathcal{S}_{\mathbb{Z}}^{*}\right|_{\left\{\sigma_{\mathbb{Z}}=0\right\}}\right)
$$

(which is described further at the beginning of $\S 6.2$ ), is nowhere vanishing. Thus under the relative orientation

$$
\operatorname{Hom}\left(\operatorname{det} T \operatorname{Gr}(d, n), \operatorname{det} \oplus_{j=1}^{n-d} \mathcal{S}_{\mathbb{Z}}^{*}\right) \cong L^{\otimes 2},
$$

we have that Jac $\sigma$ is a nowhere-vanishing section of the restriction of $L^{\otimes 2}$. Thus $\left\langle\mathrm{Jac} \sigma_{k}\right\rangle$ is either $\langle-1\rangle$ or $\langle 1\rangle$; but $\langle-1\rangle=1$ by assumption. Since $n\left(\oplus_{j=1}^{n-d} \mathcal{S}_{k}^{*}, \operatorname{EM}(\mathrm{W})\right)=\left\langle\operatorname{Jac} \sigma_{k}\right\rangle$ (compare Example 2.21), this proves the claim. 


\subsection{An arithmetic count of the $d$-planes on a complete intersection in projective space}

A complete intersection of hypersurfaces

$$
X=\left\{F_{1}=F_{2}=\cdots=F_{j}=0\right\} \subset \mathbb{P}^{n}
$$

with $F_{i}$ of degree $n_{i}$ gives rise to a section $\sigma$ of $V=\oplus_{i=1}^{j} \operatorname{Sym}^{n_{i}} \mathcal{S}^{*}$, defined by $\sigma(\mathbb{P} L)=$ $\left.\oplus_{i=1}^{j} F_{i}\right|_{L}$, where $L$ is any $(d+1)$-dimensional linear subspace of $\mathbb{A}^{n+1}$ containing the origin and $\mathbb{P} L$ denotes the corresponding point of $\operatorname{Gr}(d, n)$. The zeros of $\sigma$ are then precisely the $d$-planes in $X$ (see, e.g., [25]). By [25, Théorèye 2.1], the closed subscheme $\{\sigma=0\}$ of $\operatorname{Gr}(d, n)$ is smooth for general $X$ and either of the expected dimension $(d+1)(n-d)-$ $\operatorname{rank} V=(d+1)(n-d)-\sum_{i=1}^{j}\left(\begin{array}{c}n_{i}+d \\ d\end{array}\right)$ or empty, with the empty case occurring exactly when one or both of $(d+1)(n-d)-\operatorname{rank} V$ and $n-2 r-j$ is less than 0 . In particular, when $(d+1)(n-d)-\operatorname{rank} V=0$, the zeros of $\sigma$ are isolated and étale over $k$ for a general complete intersection $X$. The canonical relative orientation (Remark 6.2) of $V$ determines an isomorphism $\operatorname{Hom}(\operatorname{det} T \operatorname{Gr}(d, n), \operatorname{det} V) \cong L^{\otimes 2}$ for a line bundle $L$ on $\operatorname{Gr}(d, n)$. The Jacobian determinant Jac $\sigma$ at a zero $p$ of $\sigma$ is an element of the fiber of the vector bundle $\operatorname{Hom}(\operatorname{det} T \operatorname{Gr}(d, n), \operatorname{det} V)$ at $p$. Choosing any local trivialization of $L$, we have a well-defined element $\operatorname{Jac} \Sigma(p)$ in $k(p) /\left(k(p)^{*}\right)^{2}$, which can also be computed by choosing a local trivialization of $V$ and local coordinates of $\operatorname{Gr}(d, n)$ compatible with the relative orientation and computing $\operatorname{Jac} \Sigma(p)=\operatorname{det}\left(\frac{\partial \sigma_{k}}{\partial x_{l}}\right)$.

Corollary 6.9. Let $X=\left\{F_{1}=F_{2}=\cdots=F_{j}=0\right\} \subset \mathbb{P}^{n}$ be a general complete intersection of hypersurfaces $F_{i}=0$ of degree $n_{i}$ in $\mathbb{P}_{k}^{n}$ a projective space over a field $k$. Suppose that $\sum_{i=1}^{j}\left(\begin{array}{c}n_{i}+d \\ d\end{array}\right)=(d+1)(n-d)$ and $\sum_{i=1}^{j} \frac{n_{i}}{d+1}\left(\begin{array}{c}n_{i}+d \\ d\end{array}\right)+n+1$ is even. Then

$$
\sum_{d \text {-planes } \mathbb{P} L \text { in } X} \operatorname{tr}_{k(\mathbb{P} L) / k}\langle\operatorname{Jac} \sigma(\mathbb{P} L)\rangle=\frac{n_{\mathbb{C}}+n_{\mathbb{R}}}{2}+\frac{n_{\mathbb{C}}+n_{\mathbb{R}}}{2}\langle-1\rangle,
$$

where

- $k(\mathbb{P} L)$ denotes the residue field of $\mathbb{P} L$ viewed as a point on the Grassmannian;

- $n_{\mathbb{C}}$ (resp., $n_{\mathbb{R}}$ ) is the topological Euler number of the complex (resp., real) vector bundle associated to the algebraic vector bundle $V=\oplus_{i=1}^{j} \mathrm{Sym}^{n_{i}} \mathcal{S}^{*}$ given the canonical relative orientation (Remark 6.2); and

- Jac $\sigma$ is the Jacobian determinant.

Proof. By [25, Théorème 2.1], the zeros of $\sigma$ are isolated and étale over $k$. It follows [49, p.18, Proposition 34] that for a zero of $\sigma$ corresponding to the $d$-plane $\mathbb{P} L$, the local index is computed as $\operatorname{ind}_{\mathbb{P} L}^{\mathrm{PH}} \sigma=\operatorname{tr}_{k(L) / k}\langle\operatorname{Jac} \sigma(\mathbb{P} L)\rangle$ (see $\S 2.4$ for the definition of the notation ind $\left.{ }^{\mathrm{PH}}\right)$. Thus $n^{\mathrm{PH}}(V, \sigma)=\sum_{d \text {-planes } \mathbb{P} L \text { in } X} \operatorname{tr}_{k(L) / k}\langle\mathrm{Jac} \sigma(\mathbb{P} L)\rangle$ by Definition 2.24. Corollary 6.3 computes $n^{\mathrm{PH}}(V, \sigma)$. Proposition 5.9 shows that $n_{\mathbb{R}}$ and $n_{\mathbb{C}}$ are the claimed topological Euler numbers.

Remark 6.10. Note that Corollary 6.9 is a weighted count of the dimension $d$ hyperplanes on the complete intersection $X$, depending only on $n_{1}, \ldots, n_{j}$ and not on the choice of polynomials $F_{1}, \ldots, F_{j}$ as long as these are chosen generally. 
Example 6.11. Examples where Corollary 6.9 applies include lines on a degree $2 n-$ 1 hypersurface of dimension $n$, 3-planes on a degree $d$ hypersurface of dimension $2+$ $\frac{1}{3}\left(\begin{array}{c}d+3 \\ 3\end{array}\right)$ when this is an integer, and lines on a complete intersection of two degree $n-2$ polynomials in $\mathbb{P}^{n}$ for $n$ odd.

Matthias Wendt's oriented Schubert calculus shows that enriched intersections of Schubert varieties are determined by the $\mathbb{R}$ and $\mathbb{C}$ realizations in the same manner $[74$, Theorem 8.6], as well as giving enumerative applications [74, Section 9].

For $d=j=1$ and $n_{1}=3$, Corollary 6.3 is work of Kass and the second author [49] over a general field. For $d=1$ and general $j$ and $n_{i}$, it is work of M. Levine [54] over a perfect field of characteristic either 0 or prime to 2 and the odd $n_{i}$ s. Our result eliminates the assumption on the characteristic and generalizes to arbitrary relatively orientable $d, n, n_{1}, \ldots, n_{j}$.

In order to obtain an enumerative theorem whose statement is independent of $\mathbb{A}^{1}$-homotopy theory, one needs an arithmetic-geometric interpretation of the local indices:

Question 6.12. Can the local indices $\operatorname{ind}_{\mathbb{P} L}^{\mathrm{PH}} \sigma=\operatorname{tr}_{k(L) / k}\langle\mathrm{Jac} \sigma(\mathbb{P} L)\rangle$ be expressed in terms of the arithmetic geometry of the d-plane $\mathbb{P} L$ on $X$ ?

Such expressions are available over $\mathbb{R}$ for $d=j=1$ [36], and over a field $k$ of characteristic not 2 for lines on a cubic surface [49], lines on a quintic 3-fold [66], and points on a complete intersection of hypersurfaces [58]. S. Pauli has interesting observations on such results for lines on the complete intersection of two cubics in $\mathbb{P}^{5}$ : dropping the assumption that the zeros of $\sigma$ are isolated, she computes contributions from infinite families of lines on a quintic 3-fold in some cases [66]. An alternative point of view in terms of (S)pin structures for $d=j=1$ and $n_{1}=5$, as well as computations of the real Euler number, is discussed in [70, Example 1.6, Theorem 8.8].

Example 6.13. The computations of the Euler classes of $\mathbb{C}$ and $\mathbb{R}$-points in Finashin and Kharlamov's paper [35, p. 190] imply the following enriched counts of 3-planes on hypersurfaces over any field $k$ :

We have $n\left(\operatorname{Sym}^{3} \mathcal{S}^{*} \rightarrow \operatorname{Gr}(3,8)\right)=160,839\langle 1\rangle+160,650\langle-1\rangle$ corresponding to an enriched count of 3-planes in a 7-dimensional cubic hypersurface. Namely, for a general degree 3 polynomial $F$ in nine variables, the corresponding cubic hypersurface $X \subset \mathbb{P}^{8}$ contains finitely many 3-planes, as already discussed, and

$$
\sum_{3 \text {-planes } P \subset X} \operatorname{tr}_{k(L) / k}\left\langle\operatorname{Jac} \sigma_{F}(P)\right\rangle=160,839\langle 1\rangle+160,650\langle-1\rangle,
$$

where $\sigma_{F}$ is the section of $\operatorname{Sym}^{3} \mathcal{S}^{*}$ defined by $\sigma_{F}[P]=\left.F\right|_{P}$.

Similarly, $n\left(\operatorname{Sym}^{5} \mathcal{S}^{*} \rightarrow \operatorname{Gr}(3,17)\right)=$

$32,063,862,647,475,902,965,720,976,420,325\langle 1\rangle$

$$
+32,063,862,647,475,902,965,683,320,692,800\langle-1\rangle
$$


corresponds to an enriched count of 3-planes in a 16-dimensional degree 5 hypersurface.

\section{Indices of sections of vector bundles and $\mathbb{A}^{1}$-degrees}

\section{1. $\mathbb{A}^{1}$-degrees}

Recall the following:

Definition 7.1 (local $\mathbb{A}^{1}$-degree). Let $S$ be a scheme, set $X \in \operatorname{Sch}_{S}$, and let $F: X \rightarrow \mathbb{A}_{S}^{n}$ be a morphism. We say that $F$ has an isolated zero at $Z \subset X$ if $Z$ is a clopen subscheme of $Z(F)$ such that $Z / S$ is finite.

Now let $X \subset \mathbb{A}_{S}^{n}$ be open and suppose that $F$ has an isolated zero at $Z \subset X$. We define the local $\mathbb{A}^{1}$-degree of $F$ at $Z$,

$$
\operatorname{deg}_{Z}(F) \in\left[\mathbb{P}^{n} / \mathbb{P}^{n-1}, \mathbb{P}^{n} / \mathbb{P}^{n-1}\right]_{\mathcal{S H}(S)} \simeq[\mathbb{1}, \mathbb{1}]_{\mathcal{S H}(S)}
$$

as the morphism corresponding to the unstable map

$$
\mathbb{P}^{n} / \mathbb{P}^{n-1} \rightarrow \mathbb{P}^{n} / \mathbb{P}^{n} \backslash Z \simeq X / X \backslash Z \hookrightarrow X / X \backslash Z(F) \stackrel{F}{\rightarrow} \mathbb{A}^{n} / \mathbb{A}^{n} \backslash 0 \simeq \mathbb{P}^{n} / \mathbb{P}^{n-1}
$$

Here we use the assumption that $Z(F) \simeq Z \amalg Z^{\prime}$, and hence $X / X \backslash Z(F) \simeq X / X \backslash Z \amalg$ $X / X \backslash Z^{\prime}$.

Example 7.2. If $S=\operatorname{Spec}(k)$ is the spectrum of a field, then an isolated zero $z \in \mathbb{A}_{k}^{n}$ of $F: \mathbb{A}_{k}^{n} \rightarrow \mathbb{A}_{k}^{n}$ in the usual sense is also an isolated zero $\{z\} \subset \mathbb{A}_{k}^{n}$ in the foregoing sense, and $\operatorname{deg}_{z}(F) \in \mathrm{GW}(k)$ is the usual local $\mathbb{A}^{1}$-degree of [48, Definition 11].

Lemma 7.3. Set $X, Y \in \mathrm{Sm}_{S}$ and let $(Z, U, \phi, g)$ be an equationally framed correspondence from $X$ to $Y$ [30, Definition 2.1.2]; in other words, $Z \subset \mathbb{A}_{X}^{n}, U$ is an étale neighborhood of $Z, g: U \rightarrow Y$, and $\phi: U \rightarrow \mathbb{A}^{n}$ is a framing of $Z$. Then the following two morphisms are stably homotopic:

$$
\begin{gathered}
T^{n} \wedge X_{+} \simeq\left(\mathbb{P}^{1}\right)^{\wedge n} \wedge X_{+} \rightarrow\left(\mathbb{P}^{1}\right)_{X}^{\times n} /\left(\mathbb{P}^{1}\right)_{X}^{\times n} \backslash Z \simeq U / U \backslash Z \stackrel{\phi, g}{\longrightarrow} T^{n} \wedge Y_{+} \\
T^{n} \wedge X_{+} \simeq \mathbb{P}^{n} / \mathbb{P}^{n-1} \wedge X_{+} \rightarrow \mathbb{P}_{X}^{n} / \mathbb{P}_{X}^{n} \backslash Z \simeq U / U \backslash Z \stackrel{\phi, g}{\longrightarrow} T^{n} \wedge Y_{+} .
\end{gathered}
$$

Proof. For $E \in \mathcal{S H}(S)$, precomposition with the first morphism (desuspended by $T^{n}$ ) induces a map $E(Y) \rightarrow E(X)$ known as the Voevodsky transfer. Precomposition with the second map induces an 'alternative Veovedsky transfer'. It suffices (by the Yoneda lemma) to show that these transfer maps have the same effect (even just on $\pi_{0}$ ) for every $E$. In [31, Theorem 3.2.11], it is shown that the Voevodsky transfer coincides with a further construction known as the fundamental transfer. In that proof, all occurrences of $\left(\mathbb{P}^{1}\right)^{\wedge n}$ can be replaced by $\mathbb{P}^{n} / \mathbb{P}^{n-1}$; one deduces that the alternative Voevodsky transfer also coincides with the fundamental transfer.

The result follows. 
Corollary 7.4. Set $U \subset \mathbb{A}_{S}^{n}$ and let $F: U \rightarrow \mathbb{A}^{n}$ have an isolated zero along $Z \subset U$. Then $\operatorname{deg}_{Z}(F) \in[\mathbb{1}, \mathbb{1}]_{S}$ is the same as the endomorphism given by the equationally framed correspondence defined by $F$.

Proof. By definition, $\operatorname{deg}_{Z}(F)$ is given by the second morphism of Lemma 7.3, whereas the equationally framed correspondence is given by the first morphism. The result follows.

Recall also the following:

Proposition 7.5 ([31]). Let $\alpha: S \stackrel{\varpi, \tau}{\longleftarrow} Z \rightarrow S$ be a tangentially framed correspondence from $S$ to $S$ over $S$. Then the trivialization $\tau$ of $L_{Z / S}$ induces a transfer map

$$
\varpi_{*}:[\mathbb{1}, \mathbb{1}]_{Z} \rightarrow[\mathbb{1}, \mathbb{1}]_{S}
$$

where $\varpi: Z \rightarrow S$ is the projection. The endomorphism of $\mathbb{1}_{S}$ corresponding to $\alpha$ is given by $\varpi_{*}(1)$.

\subsection{Main result}

Let $X / S$ be smooth and $V / X$ a relatively oriented vector bundle with very nondegenerate section $\sigma$ and zero scheme $Z$ (which is thus finite over $S$ ). Let $Z^{\prime} \subset Z$ be a clopen component and suppose there are coordinates $\left(\psi, \varphi, \sigma^{\prime}\right)$ for $\left(V, X, \sigma, \rho, Z^{\prime}\right)$ as in Definition 2.28. Then $\sigma^{\prime}=\left(F_{1}, \ldots, F_{d}\right)$ determines a function $F: \mathbb{A}_{S}^{d} \rightarrow \mathbb{A}_{S}^{d}$, and $\varphi\left(Z^{\prime}\right)$ is an isolated zero of $F$.

Theorem 7.6. Assumptions and notations are as before. Let $E \in \mathcal{S H}(S)$ be an SLoriented ring spectrum with unit mapu: $\mathbb{1} \rightarrow$ E. Then

$$
\operatorname{ind}_{Z}(V, \sigma, \rho, E)=u_{*} \operatorname{deg}_{\varphi\left(Z^{\prime}\right)}(F) \in E^{0}(S) .
$$

Proof. By Corollary 7.4, Proposition 7.5, and $\S 4.2 .2$, we have $u_{*} \operatorname{deg}_{\varphi\left(Z^{\prime}\right)}(F)=$ $\operatorname{ind}_{Z}\left(\sigma^{\prime}, o^{\prime}, E\right)$, where $o^{\prime}$ is the canonical relative orientation of $\mathcal{O}_{\mathbb{A}^{n}}^{n} / \mathbb{A}^{n}$. The result now follows from Proposition 3.13.

Example 7.7. Suppose $S=\operatorname{Spec}(k)$ is a field. Then for well-chosen $E$ (e.g., $E=\mathrm{KO}$ or $E=\mathrm{H} \tilde{\mathbb{Z}})$, the unit map

$$
u_{*}: \mathrm{GW}(k) \simeq[\mathbb{1}, \mathbb{1}]_{\mathcal{S H}(k)} \rightarrow E^{0}(k)
$$

is an isomorphism. We deduce that $\operatorname{ind}_{z}(V, \sigma, \rho, E)$ is essentially the same as $\operatorname{deg}_{\varphi(z)}(F)$.

\section{Euler numbers in KO-theory and applications}

As explained in $\S \S 3.3$ and 5.3, we have the motivic ring spectrum KO related to Hermitian $K$-theory made homotopy invariant, and associated theories of Euler classes and Euler numbers. Using (for example) the construction in Appendix A, we can define KO even if $1 / 2 \notin S$. There is still a map $\mathrm{GW}(S) \rightarrow \mathrm{KO}(S)$, but we do not know if this is an equivalence, even if $S$ is regular (although we do know this if $S$ is regular and $1 / 2 \in S$ ). 
Example 8.1. Let $V \rightarrow X$ be a vector bundle and $\sigma$ a section. Suppose $1 / 2 \in S$. Then the refined Euler class of $V$ in KO-theory is given by $e(V, \sigma, \mathrm{KO})=[K(V, \sigma)]$, the class of the Koszul complex. Indeed, via Remark 5.15 it suffices to show the analogous result for Thom classes, which is stated at [56, p. 34].

Recall that for any lci morphism $f$ we put $\widetilde{\omega}_{f}=\widetilde{\operatorname{det}} L_{f}$ and $\omega_{f}=\operatorname{det} L_{f}$. We show in Proposition B.1 that for $f: X \rightarrow Y$ an lci morphism, we have $f^{!}(\mathcal{O}) \simeq \widetilde{\omega}_{f}$. Via Proposition B.1, coherent duality (i.e., the adjunction $f_{*} \dashv f^{!}$) thus supplies us with a canonical trace map

$$
\eta_{f}: f_{*} \widetilde{\omega}_{f} \simeq f_{*} f^{!} \mathcal{O}_{Y} \rightarrow \mathcal{O}_{Y} \in D(Y)
$$

provided that $f$ is also proper. We expect that this can be used to build a map

$$
\hat{f}_{*}: \mathrm{GW}\left(X, f^{!} \mathcal{L}\right) \rightarrow \mathrm{GW}(Y, \mathcal{L})
$$

and moreover that the following diagram commutes:

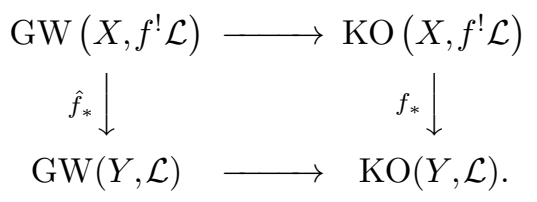

If we assume that $1 / 2 \in S$ and replace GW by W and KO by KW, then maps $\hat{f}_{*}$ can be defined and studied using the ideas from $§ 2.2$ (see also [22]). Levine and Raksit [56] show that the (modified) diagram commutes, provided that $X$ and $Y$ are smooth over a common base with $1 / 2 \in S$.

If instead we assume that $f$ is finite syntomic, then the analogous result is proved (for $\mathrm{GW} \rightarrow \mathrm{KO}$ and without $1 / 2 \in S$ ) in Corollary A.4. This is the only case we shall use in the rest of this section. Recall the construction of the Scheja-Storch form $\langle-\mid-\rangle^{\mathrm{SS}}$ from Definition 2.20.

Corollary 8.2. Set $X \in \mathrm{Sm}_{S}$, let $V / X$ be a relatively oriented vector bundle with a very nondegenerate section $\sigma$, and let $Z$ be a clopen component of the zero scheme $Z(\sigma)$. Suppose there exist coordinates $\left(\psi, \varphi, \sigma^{\prime}\right)$ around $Z$, as in Definition 2.28. Then

$$
\operatorname{ind}_{Z}(\sigma, \rho, \mathrm{KO})=\left[\langle-\mid-\rangle^{\mathrm{SS}}\left(\varphi(U), \sigma^{\prime}, S\right)\right] \in \mathrm{KO}^{0}(S)
$$

Proof. By Proposition 3.13, we may assume that $\psi=$ id and so on; so in particular, $X \subset \mathbb{A}_{S}^{n}$. The result now follows from the identification of the transfers in Corollary A.4 (telling us that the index is given by the trace form from coherent duality) and Theorem 2.18 (identifying the coherent duality form with the Scheja-Storch form).

Corollary 8.3. Let $S$ be regular semilocal scheme over a field $k$ of characteristic $\neq 2$.

(1) Let $\varpi: S^{\prime} \rightarrow S$ be a finite syntomic morphism and $\tau$ a trivialization of $L_{\varpi} \in K\left(S^{\prime}\right)$. Then the associated endomorphism of the sphere spectrum over $S$ is given under the isomorphism $[\mathbb{1}, \mathbb{1}]_{S} \simeq G W(S)$ of $[10$, Theorem 10.12] by the symmetric bilinear 
form

$$
\varpi_{*}\left(\mathcal{O}_{S^{\prime}}\right) \otimes \varpi_{*}\left(\mathcal{O}_{S^{\prime}}\right) \rightarrow \varpi_{*}\left(\mathcal{O}_{S^{\prime}}\right) \stackrel{\operatorname{det}(\tau)}{\simeq} \varpi_{*}\left(\omega_{S^{\prime} / S}\right) \stackrel{\eta_{\varpi}}{\longrightarrow} \mathcal{O}_{S}
$$

(2) Let $U \subset \mathbb{A}_{S}^{n}$ be open and define $F: U \rightarrow \mathbb{A}^{n}$ to have an isolated zero along $Z \subset U$. Then

$$
\operatorname{deg}_{Z}(F)=\left[\langle-\mid-\rangle^{\mathrm{SS}}(U, F, S)\right] \in[\mathbb{1}, \mathbb{1}]_{S} \simeq G W(S) .
$$

Proof. The proof of [10, Theorem 10.12] shows that the unit mapu: $\mathbb{1} \rightarrow$ KO induces the isomorphism $[\mathbb{1}, \mathbb{1}]_{S} \simeq G W(S)$. (1) is an immediate consequence of Proposition 7.5, Lemma 4.6(1), and Corollary A.4. Via Corollary 7.4 and Theorem 2.18, (2) is a special case of (1). This concludes the proof.

Corollary 8.4. Let $X$ be essentially smooth over a field $k$ of characteristic $\neq 2$. Let $\varpi: X^{\prime} \rightarrow X$ be a finite syntomic morphism, and suppose an orientation $\omega_{X^{\prime} / X} \stackrel{\rho}{\simeq} \mathcal{L}^{\otimes 2}$. Consider the induced transfer

$$
\varpi_{*}: \mathrm{H} \tilde{\mathbb{Z}}^{0}\left(X^{\prime}\right) \stackrel{\rho}{\simeq} \mathrm{H} \tilde{\mathbb{Z}}^{0}\left(X^{\prime}, \omega_{X^{\prime} / X}\right) \rightarrow \mathrm{H}^{0}(X) \simeq \underline{G W}(X) .
$$

Then $\varpi_{*}(1)$ is given by the image in $\underline{G W}(X)$ of the symmetric bilinear form on

$$
\varpi_{*}(\mathcal{L}) \otimes \varpi_{*}(\mathcal{L}) \rightarrow \varpi_{*}\left(\mathcal{L}^{\otimes 2}\right) \stackrel{\rho}{\simeq} \varpi_{*}\left(\omega_{X^{\prime} / X}\right) \stackrel{\eta_{\varpi}}{\longrightarrow} \mathcal{O}_{X} .
$$

Proof. Using the unramifiedness of $G W$ [60, Lemma 6.4.4], we may assume that $X$ is the spectrum of a field. Then $X^{\prime}$ is semilocal, so $\mathcal{L} \simeq \mathcal{O}$ and we obtain (up to choosing such an isomorphism) $\omega_{X^{\prime} / X} \stackrel{\rho^{\prime}}{\simeq} \mathcal{O}$. Since $L_{X^{\prime} / X}$ has constant rank (namely 0), it follows from [17, Lemma 1.4.4] that $L_{X^{\prime} / X} \simeq 0 \in K\left(X^{\prime}\right)$. The set of homotopy classes of such trivializations is given by $K_{1}\left(X^{\prime}\right)$, which maps surjectively (via the determinant) onto $\mathcal{O}^{\times}\left(X^{\prime}\right)$. It follows that there exists a trivialization $\tau: 0 \simeq L_{X^{\prime} / X} \in K\left(X^{\prime}\right)$ such that $\operatorname{det}(\tau)=\rho^{\prime}$. Hence we have a commutative diagram

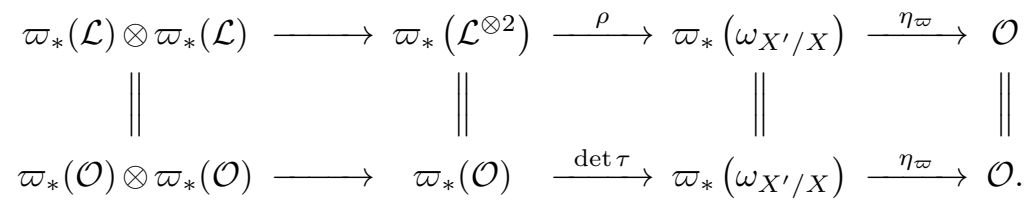

It follows from Corollary 8.3 that the bottom row is the form $\varpi_{*}(1)$ arising from the orientation $\rho$; by what we just said, this is the same as the top row, which is the form we were supposed to obtain.

This concludes the proof.

We also point out the following variant:

Corollary 8.5. Let $l / k$ be a finite extension of fields, $1 / 2 \in k$. Then Morel's absolute transfer [61, §5.1] $\operatorname{tr}_{l / k}: G W\left(l, \omega_{l / k}\right) \rightarrow G W(k)$ is given as follows: Let $\phi: V \otimes_{l} V \rightarrow l$ be an element of $G W(l), \alpha \in \omega_{l / k}^{\times}$. Then

$$
\operatorname{tr}_{l / k}(\phi \otimes \alpha)=\left[V \otimes_{k} V \rightarrow V \otimes_{l} V \stackrel{\phi}{\rightarrow} l \stackrel{\alpha}{\simeq} \omega_{l / k} \stackrel{\eta_{l / k}}{\rightarrow} k\right],
$$


where $\eta_{l / k}: \omega_{l / k} \rightarrow k$ is the (k-linear) trace map of coherent duality (see $\S B .1$ ).

Proof. This is immediate from [31, Proposition 4.3.17] (telling us that Morel's transfer coincides with the one from $\S 4.2 .4)$ and Corollary A.4.

Remark 8.6. Corollary 8.3(2) generalizes the main result of [48], at least for fields of characteristic $\neq 2$.

Remark 8.7. We expect that all of the results in this section extend to fields of characteristic 2 as well. This should be automatic as soon as KO is shown to represent GW in this situation (over regular bases, say).

\section{Appendix A. KO via framed correspondences}

In this section we will construct a strong orientation on $\mathrm{KO}$ and identify some of the transfers. We would like to thank M. Hoyois for communicating these results to us. For another approach to parts of the results in this section, see [57].

We will make use of the technology of framed correspondences [30]. We write $\operatorname{Corr}^{\mathrm{fr}}(S)$ for the symmetric monoidal $\infty$-category of smooth $S$-schemes and tangentially framed correspondences. Denote by FSyn ${ }^{\text {or }} \in \operatorname{CAlg}\left(\mathcal{P}_{\Sigma}\left(\operatorname{Corr}^{\mathrm{fr}}(S)\right)\right)$ the stack of finite syntomic schemes $Y / X$ together with a choice of trivialization $\operatorname{det} L_{Y / X} \simeq \mathcal{O}$, with its standard structure of framed transfers. This is constructed in [32, Example 3.3.4].

Write Bil $\in \operatorname{CAlg}\left(\mathcal{P}_{\Sigma}\left(\operatorname{Sch}_{S}\right)\right)$ for the presheaf sending $X$ to the 1-groupoid of pairs $(V, \phi)$ with $V \rightarrow X$ a vector bundle and $\phi: V \otimes V \rightarrow \mathcal{O}_{X}$ a nondegenerate, symmetric bilinear form. The commutative monoid structure is given by $\otimes$. If $f: X \rightarrow Y$ is a finite syntomic morphism, then a choice of trivialization $\widetilde{\operatorname{det}} L_{f} \simeq \omega_{f} \simeq \mathcal{O}_{X}$ induces an additive $\operatorname{map} \hat{f}_{*}: \operatorname{Bil}^{\simeq}(X) \rightarrow \operatorname{Bil}^{\simeq}(Y)$ (see, e.g., $\left.\S 2.2\right)$.

Theorem A.1. There exists a lift Bil $\in \operatorname{CAlg}\left(\mathcal{P}_{\Sigma}\left(\operatorname{Corr}^{\mathrm{fr}}(S)\right)\right)$ with the transfers given by maps of the form $\hat{f}_{*}$, together with a morphism $\mathrm{FSyn}^{\text {or }} \rightarrow \operatorname{Bil} \in \operatorname{CAlg}\left(\mathcal{P}_{\Sigma}\left(\operatorname{Corr}^{\mathrm{fr}}(S)\right)\right)$.

The morphism FSyn ${ }^{\text {or }} \rightarrow$ Bil is informally described as follows: a pair $(f: X \rightarrow Y$ finite syntomic, $\left.\omega_{f} \simeq \mathcal{O}\right)$ is sent to $f_{*}(\mathcal{O})$, where $\mathcal{O} \in \operatorname{Bil}(X)$ denotes the vector bundle $\mathcal{O}_{X}$ with its canonical symmetric bilinear pairing $\mathcal{O}_{X} \otimes \mathcal{O}_{X} \rightarrow \mathcal{O}_{X}$.

Proof. Denote by $K^{\circ} \in \mathcal{P}_{\Sigma}\left(\operatorname{Sm}_{S}\right)$ the rank 0 part of the $K$-theory presheaf and by $\operatorname{Corr}^{\mathrm{fr}}\left(\left(\mathrm{Sm}_{S}\right)_{/ K^{\circ}}\right)$ the subcategory of the category constructed in [32, §B] on objects $(X, \xi)$ with $X \in \mathrm{Sm}_{S}$, $\xi$ of rank 0 , and morphisms those spans whose left leg is finite syntomic. There are symmetric monoidal functors $\gamma:\left(\operatorname{Sm}_{S}\right) / K^{\circ} \rightarrow \operatorname{Corr}^{\mathrm{fr}}\left(\left(\operatorname{Sm}_{S}\right) / K^{\circ}\right)$ and $\delta: \operatorname{Corr}^{\mathrm{fr}}(S) \rightarrow \operatorname{Corr}^{\mathrm{fr}}\left(\left(\mathrm{Sm}_{S}\right) / K^{\circ}\right)$.

We first lift Bil to $\mathcal{P}_{\Sigma}\left(\operatorname{Corr}^{\mathrm{fr}}\left(\left(\operatorname{Sm}_{S}\right) / K^{\circ}\right)\right)$; we let $\operatorname{Bil}(X, \xi)$ be the 1-groupoid of vector bundles with a symmetric bilinear form for the duality $\operatorname{Hom}(-, \operatorname{det} \xi)$. Since Bil is 1truncated, we only need to specify a finite number of coherence homotopies, so this can be done by hand. Since $\delta$ is symmetric monoidal, $\delta^{*}$ is lax symmetric monoidal, and hence $\delta^{*}($ Bil $)$ produces the desired lift. 
Let $K^{\prime} \rightarrow K^{\circ}$ denote the fiber of the determinant map; in other words, this is the rank 0 part of $K^{\mathrm{SL}}$. This defines an object of $\operatorname{CAlg}\left(\mathcal{P}_{\Sigma}\left(\operatorname{Sm}_{S}\right)_{/ K^{\circ}}\right) \simeq \operatorname{CAlg}\left(\mathcal{P}_{\Sigma}\left(\left(\operatorname{Sm}_{S}\right)_{/ K^{\circ}}\right)\right)$ and $\gamma K^{\prime} \simeq$ FSyn $^{\text {or }}$ [32, Example 3.3.4 and after Example 3.3.6]. To conclude the proof, it thus suffices to construct a map $K^{\prime} \rightarrow \gamma^{*}(\mathrm{Bil}) \in \mathrm{CAlg}\left(\mathcal{P}_{\Sigma}\left(\operatorname{Sm}_{S}\right)_{/ K^{\circ}}\right)$. Again, this needs only a finite number of coherences; the desired map sends $(\xi, \phi)$ with $\xi \in K^{\circ}(X)$ and $\phi: \operatorname{det}(\xi) \simeq \mathcal{O}_{X}$ to $\left(\mathcal{O}, \phi^{\prime}\right)$, where $\phi^{\prime}: \mathcal{O} \otimes \mathcal{O} \simeq \mathcal{O} \stackrel{\phi}{\simeq} \operatorname{det} \xi$.

Since group completion and Zariski localization commute with the forgetful functor $\mathcal{P}_{\Sigma}\left(\operatorname{Corr}_{S}^{\mathrm{fr}}\right) \rightarrow \operatorname{CMon}\left(\mathcal{P}_{\Sigma}\left(\operatorname{Sm}_{S}\right)\right) \rightarrow \mathcal{P}_{\Sigma}\left(\operatorname{Sm}_{S}\right)$ [30, Proposition 3.2.14], we deduce that $\left(L_{\mathrm{Zar} B i l}{ }^{g p}\right)(X) \simeq \mathrm{KO}(X)$ [42, Definitions 1.5 and 2.2]; we denote this presheaf by $\mathrm{GW}$. There is thus a canonical Bott element $\beta \in\left[T^{4}, \mathrm{GW}\right]$.

Proposition A.2. There is a canonical equivalence $\left(\Sigma_{\mathrm{fr}}^{\infty} \mathrm{GW}\right)\left[\beta^{-1}\right] \simeq \mathrm{KO}$, at least if $1 / 2 \in S$.

Proof. The spectrum $\left(\Sigma_{\mathrm{fr}}^{\infty} \mathrm{GW}\right)\left[\beta^{-1}\right] \in \mathcal{S H}^{\mathrm{fr}}(S)$ can be modeled by the framed $T^{4}$ prespectrum $(\mathrm{GW}, \mathrm{GW}, \ldots)$ with the bonding maps given by multiplication by $\beta$. Under the equivalence $\mathcal{S} \mathcal{H}^{\mathrm{fr}}(S) \simeq \mathcal{S H}(S)$ [45], this corresponds to the same prespectrum with transfers forgotten. This is KO by definition.

In particular, we have constructed an $\mathcal{E}_{\infty}$-structure on KO.

Corollary A.3. There is a morphism $\mathrm{MSL} \rightarrow \mathrm{KO} \in \mathrm{CAlg}(\mathcal{S H}(S))$, at least if $1 / 2 \in S$.

Proof. Take the morphism $\Sigma_{\mathrm{fr}}^{\infty} \mathrm{FSyn}^{\text {or }} \rightarrow \Sigma_{\mathrm{fr}}^{\infty} \mathrm{Bil} \rightarrow \Sigma_{\mathrm{fr}}^{\infty} \mathrm{GW} \rightarrow \mathrm{KO}$ and use the fact that $\Sigma_{\mathrm{fr}}^{\infty} \mathrm{FSyn}^{\text {or }} \simeq \mathrm{MSL}[32$, Theorem 3.4.3(i)].

Corollary A.4. Let $f: Z \rightarrow S$ be a finite syntomic morphism, with $1 / 2 \in S, \tau: L_{Z / S} \simeq$ $0 \in K(Z)$ a trivialization, and $\phi: V \otimes V \rightarrow \mathcal{O}_{Z}$ be a nondegenerate, symmetric bilinear form (defining an element $[\phi] \in \mathrm{KO}^{0}(Z)$ ). Then

$$
\left[\hat{f}_{*}(\phi)\right]=f_{*}([\phi]) \in \mathrm{KO}^{0}(S)
$$

Here $f_{*}$ denotes the transfer arising from the six-functors formalism, and $\hat{f}_{*}$ denotes the transfer constructed before using coherent duality.

Proof. We first give a simplified proof assuming that $S$ is affine. Denote by $\mathrm{KO}^{\mathrm{fr}} \in$ $\mathcal{S} \mathcal{H}^{\text {fr }}(S)$ a lift of KO. By [31, Theorem 3.3.10], for any morphism $p: Z \rightarrow Y$ with $Y \in \operatorname{Sm}_{S}$ and a form $\psi$ on $Y$, the six-functors transfer of $p^{*}([\psi])$ along $f$ coincides with the framed transfer of $[\psi]$ along the correspondence $S \stackrel{\tau}{\leftarrow} Z \rightarrow Y$. By Proposition A.2, we can take 
$\mathrm{KO}^{\mathrm{fr}}=\Sigma_{\mathrm{fr}}^{\infty} \mathrm{GW}\left[\beta^{-1}\right]$; it follows that there is a map Bil $\rightarrow \Omega_{\mathrm{fr}}^{\infty} \mathrm{KO}^{\mathrm{fr}} \in \mathcal{P}_{\Sigma}\left(\mathrm{Corr}^{\mathrm{fr}}\right)$. We thus deduce that

$$
\hat{f}_{*}\left(p^{*} \psi\right)=f_{*}\left(p^{*}[\psi]\right) \in \mathrm{KO}^{0}(S) .
$$

The result would follow if there exist $p, \psi$ with $p^{*} \psi=\phi$. Under our simplifying assumption that $S$ is affine, this is always the case [32, Proposition A.0.4 and Example A.0.6(5)].

To deal with the general case, we begin with some constructions. Given $F \in \mathcal{P}\left(\operatorname{Sm}_{S}\right)$, denote by $f^{*} F \in \mathcal{P}\left(\mathrm{Sm}_{Z}\right)$ the left Kan extension. If $F$ comes from a presheaf with framed transfers, then the transfers along (base changes of) $f$ assemble to a map $f_{*} f^{*} F \rightarrow F$. Given $E \in \mathcal{S H}(S)$, we obtain $\Omega^{\infty} E \in \mathcal{P}\left(\mathrm{Sm}_{S}\right)$ and can apply this construction. On the other hand, there is a map $f_{*} \Omega^{\infty} f^{*} E \rightarrow \Omega^{\infty} E$ coming from the six-functors transfer as well as a map $f^{*} \Omega^{\infty} E \rightarrow \Omega^{\infty} f^{*} E$, and [31, Theorem 3.3.10] implies that the following diagram commutes

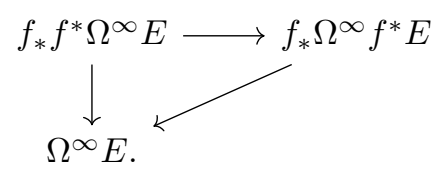

Now we continue with the proof. We have a commutative diagram

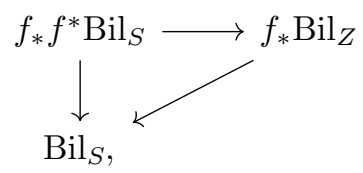

where $\operatorname{Bil}_{T} \in \mathcal{P}\left(\mathrm{Sm}_{T}\right)$ denotes the stack of symmetric bilinear forms, the map $f_{*} f^{*} \operatorname{Bil}_{S} \rightarrow$ $\mathrm{Bil}_{S}$ comes from the construction with $F=\mathrm{Bil}_{S}$, the map $f_{*} \mathrm{Bil}_{Z} \rightarrow \mathrm{Bil}_{S}$ is the transfer $\hat{f}_{*}$, and commutativity holds by construction. Applying the construction with $E=\mathrm{KO}$ and using its naturality in $F$, we obtain all in all the following commutative diagram:

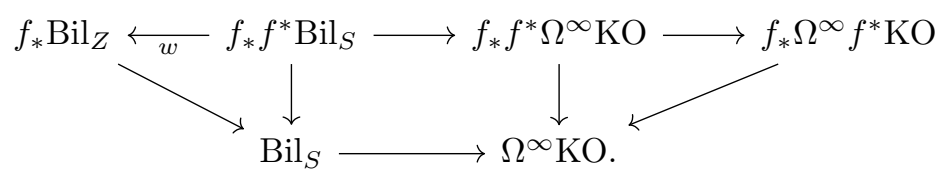

It follows from [32, Proposition A.0.4 and Example A.0.6(5)] that $f^{*} \mathrm{Bil}_{S} \rightarrow \mathrm{Bil}_{Z}$ is a Zariski equivalence, and hence the map labeled $w$ is a Nisnevich equivalence ( $f$ being finite). Since $f_{*} \Omega^{\infty} f^{*} \mathrm{KO}$ and $\Omega^{\infty} \mathrm{KO}$ are Nisnevich local, we may invert $w$ to obtain the following commutative diagram:

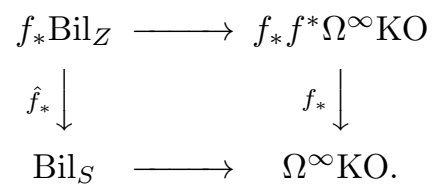

The result follows. 
Remark A.5. If $1 / 2 \notin S$, then we could define $\mathrm{KO}$ as $\left(\Sigma_{\mathrm{fr}}^{\infty} \mathrm{GW}\right)\left[\beta^{-1}\right]$. Then Corollaries A.3 and A.4 (as well as Proposition A.2) remain true. The problem is that we no longer know what theory KO represents.

\section{Appendix B. Miscellaneous}

We collect some results which we believe are well known, but for which we could not find convenient references.

\section{B.1. Cotangent complexes and dualizing complexes}

For any morphism of schemes $f: X \rightarrow Y$, there is the cotangent complex $L_{f} \in D(X)$ (see, e.g., [73, Tag 08P5]). If $f$ is lci, then $L_{f}$ is perfect [73, Tag 08SH] and hence defines a point $L_{f} \in K(X)$. Consequently, in this case we can make sense of the graded determinant $\widetilde{\operatorname{det}} L_{f} \in \operatorname{Pic}(D(X))$; this is (locally) a shift of a line bundle.

Proposition B.1. Let $f: X \rightarrow Y$ be lci. Then there is a canonical isomorphism $f^{!} \mathcal{O}_{Y} \simeq$ $\widetilde{\operatorname{det}} L_{f}$.

Recall that by our conventions, $X$ and $Y$ are separated and of finite type over some Noetherian scheme $S$; in particular, they are themselves Noetherian. We strongly believe that these assumptions are immaterial.

Proof of Proposition B.1. Both sides are compatible with passage to open subschemes of $X$. Locally on $X, f$ factors as a regular immersion followed by a smooth morphism, say $f=p i$. For either a regular immersion or a smooth morphism $g$, we have isomorphisms

$$
g^{!} \mathcal{O}_{Y} \simeq \widetilde{\operatorname{det}} L_{g}
$$

as desired [40, Definition III.2, Proposition 7.2]. For composable lci morphisms $p, i$, we have

$$
(p i)^{!}(\mathcal{O}) \simeq i^{*} p^{!}(\mathcal{O}) \otimes i^{!}(\mathcal{O}) .
$$

Similarly we have a canonical cofiber sequence $i^{*} L_{p} \rightarrow L_{p i} \rightarrow L_{i}$, and hence

$$
\widetilde{\operatorname{det}} L_{p i} \simeq i^{*} \widetilde{\operatorname{det}} L_{p} \otimes \widetilde{\operatorname{det}} L_{i}
$$

Combining formula (18), (19), and (20), we thus obtain an isomorphism

$$
\alpha_{p, i}: f^{!} \mathcal{O}_{X} \simeq i^{!} p^{!} \mathcal{O}_{X} \simeq \widetilde{\operatorname{det}} L_{f} .
$$

We have thus shown that $f^{!} \mathcal{O}_{Y}$ is locally isomorphic to $\widetilde{\operatorname{det}} L_{f}$ (via $\alpha_{p, i}$ ), and hence that $A:=f^{!} \mathcal{O}_{Y} \otimes\left(\widetilde{\operatorname{det}} L_{f}\right)^{-1}$ is an $\mathcal{O}_{X}$-module concentrated in degree 0. Exhibiting an isomorphism as claimed is the same as exhibiting $A \simeq \mathcal{O}_{X}$, or equivalently a section $a \in \Gamma(X, A)$ which locally on $X$ corresponds to an isomorphism. Since $A$ is 0 -truncated, we may construct $a$ locally. In other words, we need to exhibit a cover $\left\{U_{n}\right\}_{n}$ of $X$ and isomorphisms $\alpha_{n}:\left.\left.f^{!} \mathcal{O}_{Y}\right|_{U_{n}} \simeq \widetilde{\operatorname{det}} L_{f}\right|_{U_{n}}$ such that on $U_{n} \cap U_{m}$ we have $\alpha_{n} \simeq \alpha_{m}$. Hence, since we are claiming to exhibit a canonical isomorphism, we may do so locally on $X$. We 
may thus assume that $f$ factors as $p i$, for a smooth morphism $p: V \rightarrow Y$ and a regular immersion $i: X \rightarrow V$. We have already found an isomorphism in this situation, namely $\alpha_{p, i}$. What remains is to show that this isomorphism is independent of the choice of factorization $f=p i$.

Thus let $i^{\prime}: X \rightarrow V^{\prime}$ and $p^{\prime}: V^{\prime} \rightarrow Y$ be another such factorization. We need to show that $\alpha_{p, i}=\alpha_{p^{\prime}, i^{\prime}}$. By considering $V^{\prime} \times_{Y} V$, we may assume a commutative diagram

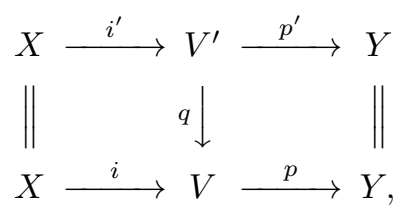

where $q$ is smooth. If in formula (20) both $f$ and $g$ are smooth, then the isomorphism arises from the first fundamental exact sequence of Kähler differentials [40, Proposition III.2.2], and hence is the same as isomorphism (19). It follows that we may assume that $p=\mathrm{id}$. The isomorphism $i^{\prime !} q^{!} \simeq i^{!}$is explained in [40, Proposition III.8.2] and reduces via formal considerations (that apply in the same way to $\widetilde{\operatorname{det}} L_{-}$) to the case of a smooth morphism with a section.

We are thus reduced to the following problem: Let $p: V \rightarrow X$ be smooth and $i: X \rightarrow V$ a regular immersion which is a section of $p$. The coherent duality formalism provides us with an isomorphism $\omega_{X / V} \otimes i^{*} \omega_{V / X} \simeq \mathcal{O}_{X}$; we need to check that this is the same as the isomorphism $\operatorname{det} L_{i} \otimes i^{*} \operatorname{det} L_{p} \simeq \operatorname{det} L_{\mathrm{id}}=\mathcal{O}_{X}$ coming from formula (20). By [40, Lemma III.8.1, Definition III.1.5], the first isomorphism arises from the second fundamental exact sequence of Kähler differentials. This is the same as the second isomorphism.

This concludes the proof.

\section{B.2. Grothendieck-Witt rings and Witt rings}

Let $R$ be a commutative ring. A symmetric space over $R$ means a finitely generated projective $R$-module $M$ together with a nondegenerate, symmetric bilinear form $\varphi: M \times$ $M \rightarrow R$. The Grothendieck group on the semiring of isomorphism classes of symmetric spaces over $R$ (with operations given by the direct sum and tensor product) is denoted $\mathrm{GW}(R)$ and called the Grothendieck-Witt ring of $R$. A symmetric space $(M, \varphi)$ is called metabolic if there exists a summand $N \subset M$ with $N=N^{\perp}$. The quotient of $\mathrm{GW}(R)$ by the subgroup (which is an ideal) generated by metabolic spaces is denoted $\mathrm{W}(R)$ and called the Witt ring of $R$.

Lemma B.2. Let $R$ be a commutative ring and $(M, \varphi)$ a symmetric space. The following are equivalent:

(1) $M$ is metabolic.

(2) $M$ contains an isotropic subspace of half rank: there exists a summand $N \subset M$ such that $\operatorname{dim} M=2 \operatorname{dim} N$ and $\left.\varphi\right|_{N}=0$.

Now suppose that all finitely generated projective $R$-modules are free of constant rankfor example, $R$ a local ring. In this case the image of $M$ in $\mathrm{GW}(R)$ is given by $n h$, where 
$\operatorname{dim} M=2 n$ and $h$ denotes the hyperbolic plane, corresponding to the matrix $\left(\begin{array}{ll}0 & 1 \\ 1 & 0\end{array}\right)$, which also satisfies $h=1+\langle-1\rangle \in \mathrm{GW}(R)$. Moreover we have a pullback square

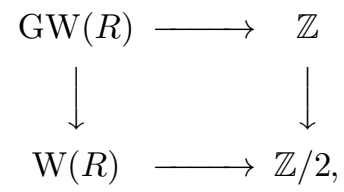

where the horizontal maps are given by rank. In particular $\mathrm{GW}(R) \rightarrow \mathrm{W}(R) \times \mathbb{Z}$ is injective.

Proof. The equivalence of (1) and (2) is [51, Corollary I.3.2]. The fact that the image in $\mathrm{GW}(R)$ is given by $n h$ follows from [51, Proposition I.3.2 and Corollary I.3.1].

Consider the symmetric space $M=\langle 1\rangle \oplus h$. Thus $M \simeq R^{3}$ has basis $e_{1}, e_{2}, e_{3}$ with $\left\langle e_{1}, e_{1}\right\rangle=1,\left\langle e_{1}, e_{2}\right\rangle=0$, and so on. Direct computation shows that $f_{1}=e_{1}+e_{2}, f_{2}=$ $e_{1}-e_{3}, f_{3}=e_{3}-e_{1}-e_{2}$ are an orthogonal basis exhibiting $M \simeq\langle 1\rangle \oplus\langle 1\rangle \oplus\langle-1\rangle$. Thus $h=1+\langle-1\rangle \in \mathrm{GW}(R)$, as claimed.

We have $\mathrm{W}(R)=\mathrm{GW}(R) / J$, where the ideal $J$ is generated by metabolic spaces. By the previous assertion, $J=\mathbb{Z} \cdot h$, and so the rank homomorphism maps $J$ isomorphically onto $2 \mathbb{Z}$. The pullback square follows formally.

Lemma B.3. Let $R$ be a local ring.

(1) Let $(M, \varphi)$ be a symmetric space over $R$. Then $M$ admits an orthogonal basis if and only if there exists $m \in M$ with $\varphi(m, m) \in R^{\times}$.

(2) $\mathrm{GW}(R)$ is generated by elements of the form $\langle a\rangle$, with $a \in R^{\times}$.

Proof. (1) If $e_{1}, \ldots, e_{n}$ is an orthogonal basis, then $\varphi\left(e_{i}, e_{i}\right)=a_{i}$, whereas $\varphi\left(e_{i}, e_{j}\right)=$ 0 for $i \neq j$; it follows now from nondegeneracy that $a_{1} \in R^{\times}$, and so the condition is necessary. Now suppose that $m \in M$ with $\varphi(m, m) \in R^{\times}$. By [59, Theorem I.3.2] we get $M \simeq R m \oplus(R m)^{\perp}$. Consider an isomorphism $M \simeq R e_{1} \oplus \cdots \oplus R e_{n} \oplus N$, where $n$ is maximal. We wish to show that $N=0$. We know that $n>0$ (by the existence of $m$ ), and if $x \in N$, then $(*) \varphi(x, x) \notin R^{\times}$(because otherwise we could split off $R x$ as before, contradicting maximality). Replacing $M$ by $e_{1} R \oplus N$, we may assume that $n=1$. Since $\left.\varphi\right|_{N}$ is nondegenerate and $R$ is local, if $N \neq 0$ we have $y, z \in N$ with $\varphi(y, z)=1$. Set $e_{1}^{\prime}=e_{1}+y$ and $f=e_{1}+\lambda z$ (with $\lambda \in R$ ). Then $\varphi\left(e_{1}^{\prime}, e_{1}^{\prime}\right)=\varphi\left(e_{1}, e_{1}\right)+\varphi(y, y) \in R^{\times}$by (*) (and using the fact that $R$ is local), and similarly $\varphi(f, f) \in R^{\times}$. On the other hand, $\varphi\left(e_{1}^{\prime}, f\right)=\varphi\left(e_{1}, e_{1}\right)+\lambda$, and hence there exists a (unique) value of $\lambda$ such that $f \in\left(R e_{1}^{\prime}\right)^{\perp}$. It follows that $M \simeq R e_{1}^{\prime} \oplus R f \oplus N^{\prime}$, in contradiction of the maximality of $n$.

(2) If $M$ is an inner product space, then $M \oplus\langle 1\rangle$ admits an orthogonal basis by (1), and hence $[M]=[M \oplus\langle 1\rangle]-\langle 1\rangle \in G W(R)$ can be expressed in terms of elements of the form $\langle a\rangle$.

Corollary B.4. We have $G \mathrm{~W}\left(\mathbb{F}_{2}\right)=\mathbb{Z}$.

Proof. It is immediate from Lemma B.3 that $\mathbb{Z} \rightarrow \mathrm{GW}\left(\mathbb{F}_{2}\right)$ is surjective. Since the rank provides a retraction, the map is also injective, hence an isomorphism. 


\section{B.3. Regular sequences}

Lemma B.5. Let $S$ be a scheme and $X \rightarrow S$ a smooth morphism, set $f_{1}, \ldots, f_{n} \in \mathcal{O}_{X}(X) k$ and put $Z=Z\left(f_{1}, \ldots, f_{n}\right)$. Assume that for all $s \in S$, either $Z_{s}$ is empty or else $\operatorname{dim} Z_{s} \leq$ $\operatorname{dim} X_{s}-n$. Then $Z \rightarrow S$ is flat, and for each $z \in Z, f_{1}, \ldots, f_{n} \in \mathcal{O}_{Z, z}$ is a strongly regular sequence. In particular, $f_{1}, \ldots, f_{n}$ is a regular sequence and $Z \rightarrow X$ is a regular immersion.

Proof. For $x \in X$ there exist affine open subschemes $x \in U \subset X, S^{\prime} \subset S$ and a factorization $U \rightarrow \mathbb{A}_{S^{\prime}}^{d} \rightarrow S^{\prime} \rightarrow S$ of $U \rightarrow S$ with $U \rightarrow \mathbb{A}_{S^{\prime}}^{n}$ étale [73, Tag 01V4]. Then $Z^{\prime}:=Z \cap U \rightarrow S^{\prime}$ still has fibers of dimension $\leq d-n$ and hence equal to $d-n$ by Krull's principal ideal theorem [73, Tag 0BBZ]. This implies that $Z^{\prime} \rightarrow S$ is a relative global complete intersection (in the sense of [73, Tag 00SP]) [30, Lemma 2.1.15], whence flat $[73,00 \mathrm{SW}]$, and $\left(f_{1}, \ldots, f_{n}\right)$ is a strongly regular sequence in $\mathcal{O}_{Z^{\prime}, z^{\prime}}$ for any $z^{\prime} \in Z^{\prime}[73$, Tag 00SV(1)]. Since $x$ was arbitrary, it follows that $Z \rightarrow S$ is flat and $\left(f_{1}, \ldots, f_{n}\right)$ form a strongly regular sequence in $\mathcal{O}_{Z, z}$ for any $z \in Z$. For the last statement, we note that if $x \in X \backslash Z$, then $f_{i} \in \mathcal{O}_{X, x}$ is a unit for some $i$, and hence $K\left(f_{\bullet}\right)_{x} \simeq 0 \simeq\left(\mathcal{O}_{Z}\right)_{x}$, whereas if $x \in Z$, then $K\left(f_{\bullet}\right)_{x} \simeq \mathcal{O}_{Z, z}$ by strong regularity [73, Tag $062 \mathrm{~F}]$.

Acknowledgments We warmly thank M. J. Hopkins for suggesting the definition of the Euler class using coherent duality. We likewise wish to thank A. Ananyevskiy and I. Panin for the reference to [52] giving the existence of Nisnevich coordinates, as well as A. Ananyevskiy, M. Hoyois, and M. Levine for useful discussions.

The second author was partially supported by National Science Foundation Awards DMS-1552730 and 2001890. The authors declare no competing interests.

\section{References}

[1] A. Ananyevskiy, SL-oriented cohomology theories, in Motivic Homotopy Theory and Refined Enumerative Geometry, Contemporary Mathematics, 745, pp. 1-19 (American Mathematical Society, Providence, RI, 2020).

[2] A. Ananyevskiy, The special linear version of the projective bundle theorem, Compos. Math. 151(3) (2015), 461-501.

[3] A. Asok and J. Fasel, Comparing Euler classes, Q. J. Math. 67(4) (2016), 603-635.

[4] M. F. АтіYAн And R. Bотt, The moment map and equivariant cohomology, Topology 23(1) (1984), 1-28.

[5] T. Bachmann, The generalized slices of hermitian k-theory, J. Topol. 10(4) (2017), 11241144.

[6] T. Bachmann, Some remarks on units in Grothendieck-Witt rings, J. Algebra 499 (2018), $229-271$.

[7] T. Bachmann, ' $\eta$-periodic motivic stable homotopy theory over dedekind domains', Preprint, 2020, arXiv:2006.02086.

[8] T. Bachmann and J. FASEl, 'On the effectivity of spectra representing motivic cohomology theories', Preprint, 2017, arXiv:1710.00594.

[9] T. Bachmann and M. J. Hopkins, ' $\eta$-periodic motivic stable homotopy theory over fields', Preprint, 2020, arXiv:2005.06778.

[10] T. Bachmann and M. Hoyois, 'Norms in motivic homotopy theory', Preprint, 2017, arXiv:1711.03061. 
[11] T. Bachmann And P. A. ØstvæR, 'Topological models for stable motivic invariants of regular number rings', Preprint, 2021, arXiv:2102.01618.

[12] J. Barge and F. Morel, Groupe de Chow des cycles orientés et classe d'Euler des fibrés vectoriels, C. R. Math. Acad. Sci. Paris 330(4) (2000), 287-290.

[13] O. Benoist and O. Wittenberg, On the integral Hodge conjecture for real varieties, I, Invent. Math. 222(1) (2020), 1-77.

[14] D. P. Berthelot, A. Grothendieck et L. Illusie, Avec la collaboration de D. Ferrand, J. P. Jouanolou, O. Jussila, S. Kleiman, M. Raynaud et J. P. Serre, Théorie des intersections et théoreme de Riemann-Roch, 225 (Springer-Verlag, Berlin-New York, 1971).

[15] C. Bethea, J. L. Kass and K. Wickelgren, Examples of wild ramification in an enriched Riemann-Hurwitz formula, in Motivic Homotopy Theory and Refined Enumerative Geometry, Contemporary Mathematics, 745, pp. 69-82 (American Mathematical Society, Providence, RI, 2020).

[16] T. Brazelton, R. Burklund, S. McKean, M. Montoro and M. Opie. The trace of the local $\mathbb{A}^{1}$-degree, Homology Homotopy Appl. 23(1) (2021), 243-255.

[17] W. Bruns and H. J. Herzog, Cohen-Macaulay Rings (Cambridge University Press, Camridge, 1998).

[18] B. Calmès, E. Dotto, Y. Harpaz, F. Hebestreit, M. Land, K. Moi, D. Nardin, T. Nikolaus and W. Steimle, 'Hermitian k-theory for stable $\infty$-categories iii', Preprint, 2020, arXiv:2009.07225.

[19] B. CAlmès and J. FASEl, 'A comparison theorem for mw-motivic cohomology', Preprint, 2017, arXiv:1708.06100.

[20] B. Calmès And J. FASEl, 'Finite Chow-Witt correspondences', Preprint, 2017, arXiv:1412.2989.

[21] B. Calmès and J. Hornbostel, Tensor-triangulated categories and dualities, Theory Appl. Categ. 22(6) (2009), 136-200.

[22] B. Calmès and J. Hornbostel, Push-forwards for Witt groups of schemes, Comment. Math. Helv. 86(2) (2011), 437-468.

[23] D.-C. Cisinski AND F. DÉGlise, Triangulated Categories of Mixed Motives, Springer Monographs in Mathematics (Springer, Cham, Switzerland, 2019).

[24] B. Conrad, Grothendieck Duality and Base Change 1750 (Springer Science \& Business Media, Springer-Verlag, Berlin, 2000).

[25] O. Debarre and L. Manivel, Sur la variété des espaces linéaires contenus dans une intersection complète, Math. Ann. 312(3) (1998), 549-574.

[26] F. DÉglise, F. Jin And A. A. Khan, 'Fundamental classes in motivic homotopy theory', Preprint, 2018, arxiv:1805.05920.

[27] D. Eisenbud, Commutative Algebra, Graduate Texts in Mathematics, 150 (SpringerVerlag, New York, 1995).

[28] D. Eisenbud and J. Harris, 3264 and All That-A Second Course in Algebraic Geometry (Cambridge University Press, Cambridge, 2016).

[29] D. Eisenbud and H. I. Levine, An algebraic formula for the degree of a $C^{\infty}$ map germ, Ann. of Math. (2) 106(1) (1977), 19-44. With an appendix by B. Teissier.

[30] E. Elmanto, M. Hoyois, A. A. Khan, V. Sosnilo and M. Yakerson, 'Motivic infinite loop spaces', Preprint, 2017, arXiv:1711.05248.

[31] E. Elmanto, M. Hoyois, A. A. Khan, V. Sosnilo and M. Yakerson, Framed transfers and motivic fundamental classes, J. Topol. 13(2) (2020), 460-500.

[32] E. Elmanto, M. Hoyois, A. A. Khan, V. Sosnilo and M. Yakerson, Modules over algebraic cobordism, Forum Math. Pi 8 (2020), e14. 
[33] J. FAsEL, The projective bundle theorem for $I^{j}$-cohomology. J. K-Theory 11(2) (2013), $413-464$.

[34] S. Finashin and V. Kharlamov, Abundance of real lines on real projective hypersurfaces, Int. Math. Res. Not. IMRN 16(16) (2013), 3639-3646.

[35] S. Finashin and V. Kharlamov, Abundance of 3-planes on real projective hypersurfaces, Arnold Math. J. 1(2) (2015), 171-199.

[36] S. Finashin and V. Kharlamov, Segre indices and Welschinger weights as options for invariant count of real lines, Int. Math. Res. Not. IMRN 6(6) (2021), 4051-4078.

[37] W. Fulton, Intersection Theory, Ergebnisse der Mathematik und ihrer Grenzgebiete (Springer-Verlag, Berlin, 1984).

[38] T. Graber and R. Pandharipande, Localization of virtual classes, Invent. Math. 135(2) (1999), 487-518.

[39] O. Gwilliam and D. Pavlov, Enhancing the filtered derived category, J. Pure Appl. Algebra 222(11) (2018), 3621-3674.

[40] R. Hartshorne, Residues and Duality, 20 (Springer-Verlag, Berlin-New York, 1966).

[41] R. Hartshorne, Algebraic Geometry, 52 (Springer-Verlag, New York-Heidelberg, 2013).

[42] J. Hornbostel, A1-representability of hermitian k-theory and Witt groups, Topology 44(3) (2005), 661-687.

[43] J. Hornbostel, M. Wendt, H. Xie and M. Zibrowius, 'The real cycle class map', Preprint, 2019, arXiv:1911.04150.

[44] M. Hoyois, The six operations in equivariant motivic homotopy theory, Adv. Math. 305 (2017), 197-279.

[45] M. Hoyois, The localization theorem for framed motivic spaces, Compos. Math. 157(1) (2021), 1-11.

[46] L. Illusie, Complexe cotangent et déformations, Number v, Lecture Notes in Mathematics, 239 (Springer-Verlag, Publisher, 1971).

[47] J. Jacobson, Real cohomology and the powers of the fundamental ideal in the Witt ring, Ann. K-Theory 2(3) (2017), 357-385.

[48] J. L. Kass and K. Wickelgren, The class of Eisenbud-Khimshiashvili-Levine is the local A1-brouwer degree, Duke Math. J. 168(3) (2019), 429-469.

[49] J. L. Kass And K. Wickelgren, An arithmetic count of the lines on a smooth cubic surface, Compos. Math. 157(4) (2021), 677-709.

[50] G. N. Khimshiashvili, The local degree of a smooth mapping, Sakharth. SSR Mecn. Akad. Moambe 85(2) (1977), 309-312.

[51] M. Knebusch, Symmetric bilinear forms over algebraic varieties, in Conference on Quadratic Forms, Queen's Papers in Pure and Applied Mathematics, 46, pp. 103-283 (Queens University, Kingston, Ontario, Canada, 1977).

[52] M.-A. Knus, Quadratic and Hermitian Forms over Rings, Grundlehren der Mathematischen Wissenschaften, 294 (Springer-Verlag, Berlin, 1991). With a foreword by I. Bertuccioni.

[53] T. Y. LAM, Introduction to Quadratic Forms over Fields, Graduate Studies in Mathematics, 67 (American Mathematical Society, Providence, RI, 2005).

[54] M. Levine, Motivic Euler characteristics and Witt-valued characteristic classes, Nagoya Math. J. 236 (2019), 251-310.

[55] M. Levine, Aspects of enumerative geometry with quadratic forms, Doc. Math. 25 (2020), $2179-2239$.

[56] M. Levine And A. Raksit, Motivic Gauss-Bonnet formulas, Algebra Number Theory 14(7) (2020), 1801-1851. 
[57] A. López-Ávila, $E_{\infty}$-Ring Structures in Motivic Hermitian K-theory, PhD thesis, Universität Osnabrück (2017).

[58] S. McKean, An arithmetic enrichment of Bézout's Theorem, Math. Ann. 379(1-2) (2021), 633-660.

[59] J. W. Milnor And D. Husemoller, Symmetric Bilinear Forms, 60 (Springer-Verlag, New York-Heidelberg, 1973).

[60] F. Morel, The stable $\mathbb{A}^{1}$-connectivity theorems, K-Theory 35(1) (2005), 1-68.

[61] F. Morel, $\mathbb{A}^{1}$-Algebraic Topology over a Field, Lecture Notes in Mathematics (Springer, Berlin, 2012).

[62] C. OKoneK And A. Teleman, Intrinsic signs and lower bounds in real algebraic geometry, J. Reine Angew. Math. 688 (2014), 219-241.

[63] I. PANin AND C. WALter, 'On the algebraic cobordism spectra msl and msp', Preprint, 2010, arXiv:1011.0651.

[64] I. PAnin And C. Walter, 'Quaternionic Grassmannians and Pontryagin classes in algebraic geometry', Preprint, 2010, arXiv:1011.0649.

[65] I. Panin and C. Walter, On the motivic commutative ring spectrum BO, Algebra $i$ Analiz 30(6) (2018), 43-96.

[66] S. PAuli, 'Quadratic types and the dynamic Euler number of lines on a quintic 3-fold', Preprint, 2020, arXiv:2006.12089.

[67] G. Scheja And U. Storch, Über Spurfunktionen bei vollständigen Durchschnitten, J. Reine Angew. Math. 278(279) (1975), 174-190.

[68] M. Schlichting, The Mayer-Vietoris principle for Grothendieck-Witt groups of schemes, Invent. Math. 179(2) (2010), 349-433.

[69] M. Schlichting and G. S. Tripathi, Geometric models for higher Grothendieck-Witt groups in $\mathbb{A}^{1}$-homotopy theory, Math. Ann. 362(3-4) (2015), 1143-1167.

[70] J. P. Solomon, Intersection Theory on the Moduli Space of Holomorphic Curves with Lagrangian Boundary Conditions, PhD thesis, https://arxiv.org/abs/math/0606429 (2006).

[71] M. SpITzWECK, A commutative $\mathbb{P}^{1}$-spectrum representing motivic cohomology over Dedekind domains, Mém. Soc. Math. Fr. (N.S.) 157(157) (2018), 110.

[72] P. SRinivasan And K. WiCKELGREn, An arithmetic count of the lines meeting four lines in $P^{3}$, Trans. Amer. Math. Soc. 374(5) (2021), 3427-3451.

[73] The Stacks Project Authors, Stacks Project, 2018, http://stacks.math.columbia.edu.

[74] M. Wendt, Oriented Schubert calculus in Chow-Witt rings of Grassmannians, in Motivic Homotopy Theory and Refined Enumerative Geometry, Contemporary Mathematics, 745, pp. 217-267 (American Mathematical Society, Providence, RI, 2020). 